\title{
Well-posedness for the Prandtl system without analyticity or monotonicity
}

\author{
${ }^{*}$ David Gérard-Varet, ${ }^{\dagger}$ Nader Masmoudi
}

\begin{abstract}
It has been thought for a while that the Prandtl system is only well-posed under the Oleinik monotonicity assumption or under an analyticity assumption. We show that the Prandtl system is actually locally well-posed for data that belong to the Gevrey class $7 / 4$ in the horizontal variable $x$. Our result improves the classical local well-posedness result for data that are analytic in $x$ (that is Gevrey class 1). The proof uses new estimates, based on non-quadratic energy functionals.
\end{abstract}

\section{Introduction}

Our concern in this paper is the well-posedness of the Prandtl system. This system, by now classical, was introduced by Prandtl in 1904 to describe an incompressible flow near a wall, at high Reynolds number. Formally, it is derived from the Navier-Stokes equation with no-slip condition:

$$
\left\{\begin{aligned}
\partial_{t} \mathbf{u}+\mathbf{u} \cdot \nabla \mathbf{u}+\nabla p-\varepsilon \Delta \mathbf{u}=0, & \mathbf{x} \in \Omega, \\
\nabla \cdot \mathbf{u}=0, & \mathbf{x} \in \Omega, \\
\left.\mathbf{u}\right|_{\partial \Omega}=0, &
\end{aligned}\right.
$$

that we consider for simplicity in $\Omega:=\mathbb{T} \times \mathbb{R}_{+}$. We recall that $\mathbf{u}(t, \mathbf{x})=(u, v)(t, x, y)$ is the velocity field of the fluid, and $p$ its pressure field. The parameter $0<\varepsilon \ll 1$ is the inverse of the Reynolds number. In the limit case $\varepsilon=0$, one is left formally with the Euler equation, for which only the impermeability condition $\left.u \cdot n\right|_{\partial \Omega}=0$ can be prescribed. Mathematically, this singular change of boundary condition generates strong gradients of the Navier-Stokes solution $\mathbf{u}^{\varepsilon}$, as $\varepsilon \rightarrow 0$. These gradients correspond to a concentration of the fluid flow in a thin zone near the wall $\partial \Omega$ : the so-called boundary layer. The understanding of the boundary layer is a great mathematical challenge, that makes the convergence of Navier-Stokes solutions to Euler ones a big open problem, even for smooth data.

To tackle this problem, Prandtl proposed in 1904 an asymptotic model for the flow, based on two different asymptotic expansions of $\mathbf{u}^{\varepsilon}$, resp. outside and inside the boundary layer:

- outside the boundary layer, no concentration should occur: one should have

$$
\mathbf{u}^{\varepsilon}(t, \mathbf{x}) \sim \mathbf{u}^{0}(t, \mathbf{x}), \quad \text { the solution of the Euler equation. }
$$

\footnotetext{
* Institut de Mathématiques de Jussieu et Université Paris 7, 175 rue du Chevaleret, 75013 Paris France

${ }^{\dagger}$ Courant Institute, NYU, 251 Mercer Street, New-York 10012. Partially supported by NSF grant DMS1211806
} 
- inside the boundary layer, $\mathbf{u}^{\varepsilon}$ should exhibit strong gradients, transversally to the boundary: more precisely, the asymptotics suggested by Prandtl is

$$
u^{\varepsilon}(t, x, y) \sim u(t, x, y / \sqrt{\varepsilon}), \quad v^{\varepsilon}(t, x, y) \sim \sqrt{\varepsilon} v(t, x, y / \sqrt{\varepsilon})
$$

where $u=u(t, x, Y)$ and $v=v(t, x, Y)$ are boundary layer profiles, depending on a rescaled variable $Y=y / \sqrt{\varepsilon}, \quad Y>0$. Note that the scale $\sqrt{\varepsilon}$ is coherent with the parabolic part of $(1.1 \mathrm{l})$.

If we plug the expansion above in (1.1) and keep the leading order terms, we derive the famous Prandtl system (denoting $Y$ instead of $y$ ):

$$
\left\{\begin{aligned}
\partial_{t} u+u \partial_{x} u+v \partial_{y} u+\partial_{x} p-\partial_{y}^{2} u & =0, \\
\partial_{y} p & =0, \\
\partial_{x} u+\partial_{y} v & =0, \\
\left.u\right|_{y=0}=\left.v\right|_{y=0} & =0, \quad \lim _{y \rightarrow+\infty} u=U, \quad \lim _{y \rightarrow+\infty} p=P,
\end{aligned}\right.
$$

where $U(t, x):=u^{0}(t, x, 0)$ and $P(t, x):=p^{0}(t, x, 0)$ are the Euler tangential velocity and pressure at the boundary. We refer to [16] for the formal derivation of the Prandtl system. The condition at $y=+\infty$ in $(1.2 \mathrm{~d})$ is a matching condition near the boundary between the boundary layer flow and the Euler flow (matched asymptotics). Note that, combining (1.2 b) with the boundary condition on $p$, we get $p \equiv P$. Hence, the pressure is not an unknown in the Prandtl model: $v$ is obtained in terms of $u$ by integrating the divergence-free condition $(1.2 \mathrm{r})$, so that $(1.2 \mathrm{a})$ is a scalar evolution equation on $u$, which is a priori much simpler than the original Navier-Stokes equation.

However, this appealing formal asymptotics raises strong mathematical issues: wellposedness of the limit Prandtl system on one hand, justification of the Prandtl asymptotics of $\mathbf{u}^{\varepsilon}$ on the other hand. The difficulty comes from numerous underlying fluid instabilities, that can invalidate the Prandtl model: we refer to [10] for a basic presentation of these aspects.

The aim of the present paper is to investigate this stability problem, from a mathematical viewpoint. We shall focus on the limit Prandtl system, namely on its well-posedness. For simplicity, we shall restrict to homogeneous data: $U=P=0$. Extension of our results to the case of constant $U$ would not raise any problem. Extension to some $U=U(t, x)$ would require some modifications, see [13] for a similar problem. Hence, we consider here the following system:

$$
\left\{\begin{array}{r}
\partial_{t} u+u \partial_{x} u+v \partial_{y} u-\partial_{y}^{2} u=0, \\
\partial_{x} u+\partial_{y} v=0 \\
\left.u\right|_{y=0}=\left.v\right|_{y=0}=0, \lim _{y=+\infty} u=0
\end{array}\right.
$$

with initial condition $\left.u\right|_{t=0}=u_{0}$.

Before stating our theorem, let us review briefly known results on the existence theory for (1.2). So far, well-posedness has been established in two settings:

- The first results go back to Oleinik [20], who obtained some local well-posedness for initial data that are monotonic with respect to $y: U>0, \partial_{y} u>0$. For such data, one can use the Crocco transform: in short, using $u$ as an independent variable instead of $y$ 
and $w:=\partial_{y} u$ as an unknown instead of $u$, one is left with a nonlinear parabolic equation on $w$, for which maximum principles are available: see [20] for details. Note that under the extra condition $\partial_{x} P \leq 0$ (favorable pressure gradient), one can go from local to global well-posedness, $c f$ [24]. From the point of view of physics, this monotonicity assumption is known to be stabilizing: it avoids the boundary layer separation, see [10].

- Without monotonicity, well-posedness has been established only locally in time, for initial data that are analytic with respect to $x$. We refer to [23, 15], and to the recent extension [13]. The assumption of analyticity can be understood as follows. By the divergence-free condition, one obtains $v=-\int_{0}^{y} \partial_{x} u$. Thus, the term $v \partial_{y} u$ in (1.3a) (seen as a functional of $u$ ) is first order in $x$. Moreover, it is not hyperbolic. For instance, let us consider the linearization of the Prandtl equation around a shear flow $\mathbf{u}=\left(U_{s}(y), 0\right)$ :

$$
\partial_{t} u+U_{s} \partial_{x} u+U_{s}^{\prime} v-\partial_{y}^{2} u=0, \quad \partial_{x} u+\partial_{y} v=0 .
$$

If we freeze the coefficients at some $y_{0}$ and compute the dispersion relation, we obtain the growth rate

$$
\sigma\left(k_{x}, k_{y}\right)=U_{s}^{\prime}\left(y_{0}\right) \frac{k_{x}}{k_{y}}-k_{y}^{2}
$$

that increases linearly with the wavenumber $k_{x}$. This kind of growth rate would prevent any well-posedness result outside the analytic setting.

However, as discussed in [11, this dispersion relation, formally obtained by freezing the coefficients, is misleading: for instance, the inviscid version of Prandtl (that is removing the $\partial_{y}^{2} u$ term ) is locally well-posed in $C^{k}$, through the method of characteristics.

In the case of the full Prandtl system (1.3), the situation is even more complex, and was addressed recently by the first author and Emmanuel Dormy in article [6] (see also [7]). This article contains a careful study of the linearized system (1.4), in the case of a non-monotonic base flow $U_{s}$ :

$$
\exists a, \quad U_{s}^{\prime}(a)=0, \quad U_{s}^{\prime \prime}(a) \neq 0 .
$$

In short, it is shown in [6] that the linear system (1.4) admits approximate solutions with growth rate

$$
\sigma\left(k_{x}\right) \sim \delta \sqrt{k_{x}}, \quad \delta>0, \quad k_{x} \gg 1
$$

Let us stress that such growing solutions result from an interplay between the lack of monotonicity of $U_{s}$ and the diffusion term $\partial_{y}^{2} u$. It is therefore coherent with the well-posedness results obtained in the monotonic case, and for the inviscid Prandtl equation.

Of course, the growth rate indicated in (1.5) yields ill-posedness of the Prandtl equation in Sobolev spaces. Still, it leaves room for well-posedness below analytic regularity. Indeed, the aim of the present paper is to prove that the Prandtl equation is locally well-posed for data that are of Gevrey class $7 / 4$ in variable $x$. Precise statements will be given in the next section. Let us already point out that the Gevrey classes $m, m>1$, contain compactly supported functions, as opposed to the Gevrey class 1 (analytic functions). Hence, stability in the Gevrey context has more physical insight that stability in the analytic context: see [19] for a similar issue.

\footnotetext{
${ }^{1}$ As mentioned recently by S. Cowley to the first author, the instability mechanism used in [6] had already been described at a formal level in $\underline{3}$.
} 


\section{Statements of the result}

Let $m \geq 1$. We recall that the Gevrey space $G^{m}(\mathbb{T})$ is the set of functions $f$ satisfying: $\exists C, \tau>0$ such that

$$
\left|f^{(j)}(x)\right| \leq C \tau^{-j}(j !)^{m}, \quad \forall j \in \mathbb{N}, x \in \mathbb{T} .
$$

For a reminder on Gevrey spaces, we refer to the paper [5] by Foias and Temam as well as to the papers [14, 4, 21] where these spaces are used. One has in particular $G^{m}(\mathbb{T})=\cup_{\tau>0} G_{\tau}^{m}(\mathbb{T})$, where

$$
G_{\tau}^{m}(\mathbb{T}):=\left\{f \in C^{\infty}(\mathbb{T}), \quad \sup _{j} \tau^{j}(j !)^{-m}(j+1)^{10}\left\|f^{(j)}\right\|_{L^{2}}<\infty\right\}
$$

is a Banach space, stable by multiplication. Note that the extra factor $(j+1)^{10}$ is useful in proving the stability by multiplication [14, 4] (the exponent 10 is arbitrary, any power greater than 1 works as well).

In the context of the Prandtl equation, functions depend not only on $x$, but also on $y$. We just require Sobolev regularity and polynomial decay with respect to the $y$ variable. For $s \in \mathbb{N}, \gamma \geq 0$, we define the spaces

$$
H_{\gamma}^{s}:=\left\{g \in H^{s}\left(\mathbb{R}_{+}\right), \quad(1+y)^{\gamma+k} g^{(k)} \in L^{2}\left(\mathbb{R}_{+}\right), k=0 \ldots s\right\}, \quad\|g\|_{H_{\gamma}^{s}}^{2}:=\sum_{k=0}^{s}\left\|(1+y)^{\gamma+k} g^{(k)}\right\|_{L^{2}}^{2} .
$$

We write $L_{\gamma}^{2}$ instead of $H_{\gamma}^{0}$. Accordingly, we introduce the space

$$
G^{m}\left(\mathbb{T} ; H_{\gamma}^{s}\right):=\cup_{\tau>0} G_{\tau}^{m}\left(\mathbb{T} ; H_{\gamma}^{s}\right)
$$

where

$$
G_{\tau}^{m}\left(\mathbb{T} ; H_{\gamma}^{s}\right):=\left\{f \in C^{\infty}\left(\mathbb{T} ; H_{\gamma}^{s}\right), \quad \sup _{j} \tau^{j}(j !)^{-m}(j+1)^{10}\left\|\partial_{x}^{j} f\right\|_{L^{2}\left(H_{\gamma}^{s}\right)}<\infty\right\} .
$$

We shall consider initial data $u_{0}$ satisfying

$$
u_{0} \in G_{\tau_{0}}^{m}\left(\mathbb{T} ; H_{\gamma-1}^{s+1}\right), \quad \omega_{0}:=\partial_{y} u_{0} \in G_{\tau_{0}}^{m}\left(\mathbb{T} ; H_{\gamma}^{s}\right)
$$

for some $m, s, \tau_{0}$ to be fixed later. Our focus will be on data that are non-monotonic with respect to $y$. More precisely, we shall assume the existence of a single curve of non-degenerate critical points:

(H) $\quad \omega_{0}(x, y)=0$ iff $y=a_{0}(x)$, for some curve $a_{0}(x)>0$, with $\partial_{y} \omega_{0}\left(x, a_{0}(x)\right)>0, \forall x$.

Without loss of generality, we will assume that $a_{0}(x)<3$. Besides, to control the behaviour of the flow at large $y$, we shall need uniform lower and upper bounds on vorticity. We assume the existence of $\sigma>0$ and $\delta>0$ such that

$$
\begin{aligned}
& \text { For all } y>3 \text {, for all } x \text {, for all } \alpha \in \mathbb{N}^{2}|\alpha| \leq 2, \\
& \qquad\left|\omega_{0}(x, y)\right| \geq \frac{2 \delta}{(1+y)^{\sigma}}, \quad\left|\partial^{\alpha} \omega_{0}(x, y)\right| \leq \frac{1}{2 \delta(1+y)^{\sigma+\alpha_{2}}} .
\end{aligned}
$$

We can now state our main result: 
Theorem 1 Let $\tau_{0}>0, s \geq 8$ even, $\gamma \geq 1, \sigma \geq \gamma+\frac{1}{2}, \delta>0$. Let $u_{0}$ satisfying

$$
u_{0} \in G_{\tau_{0}}^{7 / 4}\left(\mathbb{T} ; H_{\gamma-1}^{s+1}\right), \quad \omega_{0}:=\partial_{y} u_{0} \in G_{\tau_{0}}^{7 / 4}\left(\mathbb{T} ; H_{\gamma}^{s}\right),
$$

the compatibility condition: $\left.u_{0}\right|_{y=0}=0$, and assumptions $(\mathrm{H}),\left(\mathrm{H}^{\prime}\right)$ above. Then, there exists $T>0,0<\tau \leq \tau_{0}$ and a unique solution

$$
u \in L^{\infty}\left(0, T ; G_{\tau}^{7 / 4}\left(\mathbb{T} ; H_{\gamma-1}^{s+1}\right)\right), \quad \omega \in L^{\infty}\left(0, T ; G_{\tau}^{7 / 4}\left(\mathbb{T} ; H_{\gamma}^{s}\right)\right),
$$

of the Prandtl equation (1.3), with initial data $u_{0}$.

Remark that a solution $u$ of (1.3) with the regularity above automatically satisfies

$$
\partial_{t} u \in L^{\infty}\left(0, T ; G_{\tau^{\prime}}^{7 / 4}\left(\mathbb{T} ; H_{\gamma-1}^{s-1}\right)\right), \quad \forall \tau^{\prime}<\tau .
$$

This yields continuity in time of $u$ with values in $G_{\tau^{\prime}}^{7 / 4}\left(\mathbb{T} ; H_{\gamma-1}^{s-1}\right), \tau^{\prime}<\tau$, giving a meaning to the initial condition.

Let us point out that the solution $u$ of the theorem remains in the Gevrey space $G^{7 / 4}$ in $x$, but does not stay a priori in $G_{\tau_{0}}^{7 / 4}$ : it is likely that the exponent $\tau$ deteriorates with time.

The theorem will be the consequence of the control of some well-chosen Gevrey type norms that evolve in time. More precisely, introducing the vorticity $\omega:=\partial_{y} u$, we will control an energy of the form $E(t, \tau(t))$, with

$$
E(t, \tau):=\sum_{j=0}^{+\infty}\left(\tau^{j}(j !)^{-7 / 4}(j+1)^{10}\right)^{2}\left\|\partial_{x}^{j} \omega(t, \cdot)\right\|_{L^{2}\left(H_{\gamma}^{s}\right)}^{2}
$$

for some positive function $\tau(t)$ decreasing linearly and fast enough with $t$. Actually, it will be slightly better to consider a variant of the previous energy, namely

$$
E_{\omega}(t, \tau):=\sum_{j=0}^{+\infty}\left(\tau^{j}(j !)^{-7 / 4}(j+1)^{10}\right)^{2}\|\omega\|_{\mathcal{H}_{\gamma}^{j}}^{2},
$$

with

$$
\|\omega\|_{\mathcal{H}_{\gamma}^{j}}^{2}:=\sum_{\substack{J=\left(j_{1}, j_{2}\right) \in \mathbb{N}^{2} \\|J|=j, 0 \leq j_{2} \leq s}}\left\|(1+y)^{\gamma+j_{2}} \partial^{J} \omega(t, \cdot)\right\|_{L^{2}\left(\mathbb{T} \times \mathbb{R}_{+}\right)}^{2} .
$$

We leave it to the reader to check that: $\exists c, C>0$ such that

$$
E(t, \tau) \leq E_{\omega}(t, \tau), \quad E_{\omega}(t, \tau) \leq C_{\tau, \tau^{\prime}} E\left(t, \tau^{\prime}\right), \quad \forall 0<\tau<\tau^{\prime} .
$$

Roughly, the choice of $E_{\omega}$ upon $E$ is connected with estimates on the hyperbolic part of (1.3), for which derivatives with respect to $x$ and $y$ have the same cost.

Still, the energy $E_{\omega}$ can not be controlled in a direct manner. As mentioned in [17, there is a problem with estimating $\partial_{x}^{j} \omega$. If the $\partial_{x}^{j}$ hits the $v$ in the transport term $v \partial_{y} \omega$, we end up with $\partial_{x}^{j} v \partial_{y} \omega$, which gives an a priori loss of one full derivative and requires analytic data. This is the main trouble with the Prandtl system, already pointed out in the introduction. 
To overcome this difficulty, we shall rely on new estimates, notably inspired by the recent paper [17] by Wong and the second author. This paper is about the well-posedness of the Prandtl equations for monotonic data, that is when $u_{0}$ is increasing with $y$. As we recalled in the introduction, in this monotonic case, well-posedness was obtained by Oleinik in the 60's, using the Crocco transform. The novelty in [17] is to obtain such well-posedness result without using the Crocco transform, namely performing Sobolev estimates in the original Eulerian formulation (see also [1] where estimates on the linearized problem were done). The main point in the proof is to avoid the loss of derivative generated by the $v$-term. One key idea is the following: combining properly the velocity formulation (1.3) and the vorticity formulation $\left(\omega=\partial_{y} u\right)$

$$
\partial_{t} \omega+u \partial_{x} \omega+v \partial_{y} \omega-\partial_{y}^{2} \omega=0
$$

one is left with an equation of the form

$$
\partial_{t} g^{m w}+u \partial_{x} g^{m w}-\partial_{y}^{2} g^{m w}=\text { commutators }
$$

on the new nonlinear quantity:

$$
g^{m w}:=\omega-\frac{\partial_{y} \omega}{\omega} u=\omega \partial_{y} \frac{u}{\omega} .
$$

The main point with this new equation is that the function $v$, responsible for the loss of one $x$-derivative, does not appear. Similarly, one can write down equations on

$$
g_{j}^{m w}:=\partial_{x}^{j} \omega-\frac{\partial_{y} \omega}{\omega} \partial_{x}^{j} u
$$

that do not involve the bad term $\partial_{x}^{j} v$. Moreover, broadly speaking, one can show that the control of the family $g_{j}$ in $L^{2}$ amounts to the control of the family $\partial_{x}^{j} \omega$ in $L^{2}$. Hence, one can expect to derive a Gronwall type inequality, at the Sobolev level, using the $g_{j}$ 's (see [17]).

Of course, the main difference between the present context and the one in [17] is assumption $(\mathrm{H})$, that is the existence of a curve of critical points. This critical curve, that reads initially $y=a_{0}(x)$, with $0<a_{0}<3$, should evolve into some $y=a(t, x)$, with $0<a<3$ for small times. Differentiating the relation $\omega(t, x, a(t, x))=0$, one finds that the function $a$ should be governed by the ordinary differential equation in the variable $t$ :

$$
\partial_{t} a(t, x)+\frac{\partial_{t} \omega(t, x, a(t, x))}{\partial_{y} \omega(t, x, a(t, x))}=0, \quad a(0, x)=a_{0}(x) .
$$

The quantities $g_{j}^{m w}$ 's are not suitable in a neighborhood of this curve. As we shall see, even away from it, the $\partial_{x}^{j} \omega$ 's are not controlled by the $g_{j}^{m w}$ 's in a suitable way, due to nonlocal phenomena.

Therefore, we need to introduce an additional quantity, that somehow recovers the information lost near the critical curve. We stress that in this region, the flow should not be monotonic anymore. However, due to our non-degeneracy assumption, it should remain convex: $\partial_{y}^{2} u=$ $\partial_{y} \omega>0$, for $t$ and $y-a(t, x)$ small enough. It turns out that this kind of convexity assumption has been used in a close context, namely in the study of the hydrostatic Euler equations. These equations, set in $\mathbb{T} \times(0,1)$, read

$$
\left\{\begin{aligned}
\partial_{t} u+u \partial_{x} u+v \partial_{y} u+\partial_{x} p=0, \partial_{y} p=0 \\
\partial_{x} u+\partial_{y} v=0 \\
\left.v\right|_{y=0}=\left.v\right|_{y=1}=0 .
\end{aligned}\right.
$$


Again, for this system, there is a possible loss of $x$-derivative through the function $v$. As shown in Brenier [2], Grenier [8], the well-posedness of the hydrostatic equations requires some convexity assumption. This fact was emphasized in another recent paper by Wong and the second author [18]. Starting again from the vorticity equation,

$$
\partial_{t} \omega+u \partial_{x} \omega+v \partial_{y} \omega=0
$$

and dividing by $\sqrt{\partial_{y} \omega}$, one is left with an equation of the type

$$
\partial_{t} h^{m w}+u \partial_{x} h^{m w}+\sqrt{\partial_{y} \omega} v=\text { commutators }
$$

on $h^{m w}:=\frac{\omega}{\sqrt{\partial_{y} \omega}}$. One can then take advantage of the cancellation

$$
\int_{\mathbb{T} \times(0,1)} \sqrt{\partial_{y} \omega} v h^{m w}=\int_{\mathbb{T} \times(0,1)} v \partial_{y} u=-\int_{\mathbb{T} \times(0,1)} \partial_{y} v u=\int_{\mathbb{T} \times(0,1)} \partial_{x} \frac{|u|^{2}}{2}=0
$$

to get rid of the bad terms in $v$. Let us note that the same idea was used by Grenier in [9] to establish the stability of some characteristic boundary layers. Similar cancellations hold with higher order derivatives in $x$, through the introduction of

$$
h_{j}^{m w}:=\frac{\partial_{x}^{j} \omega}{\sqrt{\partial_{y} \omega}} .
$$

Thanks to these quantities, one can obtain local in time Sobolev estimates, like for the Prandtl equations in the monotonic case.

In our context, with regards to the previous remarks, it is tempting to replace the original energy $E_{\omega}$ by a modified one, based on functions $g_{j}$ and $h_{j}$ like in (2.3) and (2.6). To be more specific, one could think of combining two local energies: one away from the critical curve, based on the $g_{j}$ 's, and one in a neighborhood of the critical curve, based on the $h_{j}$ 's. With regards to the recent works [18, 17, one could even expect to obtain stability in Sobolev like spaces. However, such localization process is not straightforward. Indeed, the Prandtl equation, like the hydrostatic one (see recent developments by Renardy [22]) is highly nonlocal. This non-locality explains the ill-posedness of the Prandtl equation in the Sobolev setting, as can be seen from the mechanism described by the first author and Dormy [6].

At the level of the energy estimates that we will perform, this non-locality will be reflected by some annoying commutator terms. To control such bad commutators, we will use a functional of the following type:

$$
\mathcal{E}(\alpha, t, \tau):=\dot{E}_{\omega}(t, \tau)+E_{h}(t, \tau)+E_{g}^{1}(t, \tau)+\alpha E_{g}^{2}(t, \tau)
$$

where $\alpha>0$ is a parameter to be chosen later. This functional splits into four parts:

- The first part is a vorticity energy

$$
\dot{E}_{\omega}(t, \tau):=\sum_{j \in \mathbb{N}}\left(\tau^{j}(j !)^{-7 / 4}(j+1)^{10}\right)^{2}\|\omega\|_{\dot{\mathcal{H}}_{\gamma}^{j}}^{2}
$$

with

$$
\|\omega\|_{\mathcal{H}_{\gamma}^{j}}^{2}:=\sum_{\substack{J=\left(j_{1}, j_{2}\right) \in \mathbb{N}^{2} \\|J|=j, 0<j_{2} \leq s}}\left\|(1+y)^{\gamma+j_{2}} \partial^{J} \omega(t, \cdot)\right\|_{L^{2}\left(\mathbb{T} \times \mathbb{R}_{+}\right)}^{2}
$$


The difference with the original energy $E_{\omega}(t, \tau)$ is the restriction $j_{2}>0$ which means that the derivatives $\partial_{x}^{j} \omega$ are not included here. For each $j$ this energy will provide a control of all $(x, y)$ derivatives of order $j$ but $\partial_{x}^{j} \omega$. For $j=0$, one has $\|\omega\|_{\dot{\mathcal{H}}_{\gamma}^{j}}=0$, but we keep it in the sum for unity.

- The second part is a hydrostatic energy

$$
E_{h}(t, \tau):=\sum_{j \in \mathbb{N}}\left(\tau^{j}(j !)^{-7 / 4}(j+1)^{10}\right)^{2}\left\|h_{j}(t, \cdot)\right\|_{L^{2}\left(\mathbb{T} \times \mathbb{R}_{+}\right)}^{2}
$$

where

$$
h_{j}(t, x, y)=\chi(y-a(t, x)) \frac{\partial_{x}^{j} \omega}{\sqrt{\partial_{y} \omega}}(t, x, y)
$$

with $\chi=\chi(p) \in C_{c}^{\infty}(\mathbb{R})$ equal to 1 in a neighborhood of $p=0$. We take $\chi$ with small enough support, so that $\chi(y-a)$ is compactly supported in $(0,3)$, and $\partial_{y} \omega>0$ over the support of $\chi$. This truncation function corresponds to the localization near the critical curve mentioned above.

- The third part is a (first) monotonicity energy

$$
E_{g}^{1}(t, \tau)=\sum_{j \in \mathbb{N}}\left(\tau^{j}(j !)^{-7 / 4}(j+1)^{10}\right)^{2}\left\|g_{j}(t, \cdot)\right\|_{L^{2}\left(L_{\gamma}^{2}\right)}^{2}
$$

where

$$
g_{j}(t, x, y):=(\psi(y) \omega(t, x, y)+1-\psi(y))\left(\partial_{x}^{j} \omega-\frac{\partial_{y} \omega}{\omega} \partial_{x}^{j} u\right)(t, x, y)
$$

with $\psi=\psi(y) \in C_{c}^{\infty}(\mathbb{R})$, equal to 1 in an open neighborhood of $[0,3]$. Note that the truncation $\psi$ makes the quantity $g_{j}$ well-defined for all $y$, even in the neighborhood of $y=a(t, x)$. Indeed, for large $y$, it amounts to the original definition (2.3), whereas near the critical curve, it reads

$$
g_{j}(t, x, y)=\omega \partial_{x}^{j} \omega-\partial_{y} \omega \partial_{x}^{j} u .
$$

Note that the first term at the right-hand side vanishes near the critical curve, which leads to a loss of control of $\partial_{x}^{j} \omega$ in terms of $g_{j}$. As explained before, this is why we add the hydrostatic energy to the energy functional. More precisely, we will show that the sum of the vorticity energy, the hydrostatic energy, and the first monotonicity energy controls the original functional $E_{\omega}(t, \tau)$. However, we are not able to obtain a closed estimate on this sum.

- Hence, we need to add a second monotonicity energy

$$
E_{g}^{2}(t, \tau)=\sum_{j \in \mathbb{N}}\left(\tau^{j}(j !)^{-7 / 4}(j+1)^{10}\right)^{2}(j+1)^{3 / 2}\left\|\tilde{g}_{j}(t, \cdot)\right\|_{L^{2}\left(\mathbb{T} \times \mathbb{R}_{+}\right)}^{2}
$$

where

$$
\tilde{g}_{j}(t, x, y):=\partial_{x}^{j-5}\left(\omega \partial_{x}^{5} \omega-\partial_{y} \omega \partial_{x}^{5} u\right)
$$


(with convention $\partial_{x}^{k}=0$ for $k<0$ ). Let us remark that $\tilde{g}_{j}$ is close to $g_{j}$ : for instance, in the region $\psi=1$, one has $\tilde{g}_{j}=g_{j}$ up to commutator terms. Indeed, the replacement of $g_{j}$ by $\tilde{g}_{j}$ is only a technical issue, that will be explained in due course. The real keypoint in the definition of this second monotonicity energy is the extra factor $(j+1)^{3 / 2}$, creating an anisotropy in the total energy $\mathcal{E}(\alpha, t, \tau)$. Such a choice of anisotropic energy is the main feature that will allow us to prove stability estimates in the Gevrey setting, below the analytic case.

\section{A priori estimates}

The key for Gevrey well-posedness is some a priori estimate on the anisotropic energy $\mathcal{E}(\alpha, t, \tau)$ introduced in the previous section. This energy involves notably the functions $g_{j}$, see (2.12), that contains the factor $\partial_{y} \omega / \omega$. To control their behaviour for large $y$, a lower bound on $\omega$ and an upper bound on $\partial_{y} \omega$ are needed. More precisely, we shall work with vorticities $\omega$ satisfying: for all $y>3$, for all $t, x$, for all $\alpha \in \mathbb{N}^{2},|\alpha| \leq 2$,

$$
|\omega(t, x, y)| \geq \frac{\delta}{(1+y)^{\sigma}}, \quad\left|\partial^{\alpha} \omega(t, x, y)\right| \leq \frac{1}{\delta(1+y)^{\sigma+\alpha_{2}}} .
$$

Let us point out that this condition is the "all-time" version of condition (H'), the latter dealing only with the initial time. Of course, in the end, we will only assume (H') and we will need to show that such upper and lower bounds are preserved with time (up to a choice of a smaller $\delta$ ).

\section{Theorem 2 (Main a priori estimate)}

Let $T>0, \tau \geq 1, s, \gamma, \sigma$ as in Theorem 1, Let $u$ be a smooth solution of the Prandtl equation over $] 0, T]$, with a single curve of non-degenerate critical points: $y=a(t, x), 0<a<3$. Assume that the vorticity $\omega$ satisfies (3.1) over ]0,T], and that

$$
\left.\left.E_{\omega}(t, \tau) \leq M, \quad M>0, \quad \forall t \in\right] 0, T\right] .
$$

Then, there exists $\alpha>0, C>0$ such that for all $t \in] 0, T]$ :

$$
\partial_{t} \mathcal{E}(\alpha, t, \tau) \leq C \partial_{\tau} \mathcal{E}(\alpha, t, \tau) .
$$

A close look at the proof will show that the constants $\alpha$ and $C$ depend on $\tau, M, \inf a, \sup a$, $\inf _{\{y=a\}}\left|\partial_{y} \omega\right|, \inf _{|y-a| \geq \varepsilon, y \leq 3}|\omega|$ (where, for instance, $\varepsilon:=\frac{\inf _{\{y=a\}}\left|\partial_{y} \omega\right|}{2 \sup _{\mathbb{T} \times \mathbb{R}_{+}}\left|\partial_{y}^{2} \omega\right|}$ ), and the $\delta$ in (3.1).

Let us mention again that in the upper bound $a<3$ or in the condition $y>3$ in (3.1), the choice of the value 3 is purely arbitrary.

The estimate of the theorem will yield the Gevrey stability, playing on the radius $\tau$ of Gevrey regularity. Indeed, taking some time-dependent $\tau(t)$, we observe that

$$
\partial_{t} \mathcal{E}(\alpha, t, \tau(t))=\partial_{t} \mathcal{E}(\alpha, t, \tau(t))+\tau^{\prime}(t) \partial_{\tau} \mathcal{E}(\alpha, t, \tau(t)) \leq\left(C+\tau^{\prime}(t)\right) \mathcal{E}(\alpha, t, \tau(t))<0
$$

if $\tau$ is decreasing fast enough with time.

We insist that all this section is about a priori estimates. The construction of solutions will require a further approximation scheme, on which similar estimates will be shown to hold. This will be detailed in later sections. 


\subsection{Preliminaries}

In order to prove Theorem 2, we need some extra notations. For any $p \in[1,+\infty[$, we introduce the weighted $l^{p}$ space

$$
l^{p}(\tau):=\left\{\left(a_{j}\right)_{j \in \mathbb{N}}, \quad \sum_{j=0}^{+\infty}\left(\tau^{j}(j !)^{-7 / 4}(j+1)^{10}\right)^{2}\left|a_{j}\right|^{p}<+\infty\right\},
$$

with norm

$$
\left\|a_{j}\right\|_{l^{p}(\tau)}:=\left(\sum_{j=0}^{+\infty}\left(\tau^{j}(j !)^{-7 / 4}(j+1)^{10}\right)^{2}\left|a_{j}\right|^{p}\right)^{1 / p} .
$$

In particular, we have $\dot{E}_{\omega}(t, \tau)=\|\| \omega\left\|_{\mathcal{H}_{\gamma}^{j}}\right\|_{l^{2}(\tau)}^{2}$ and $\partial_{\tau} \dot{E}_{\omega}(t, \tau) \sim\left\|j^{1 / 2}\right\| \omega\left\|_{\dot{\mathcal{H}}_{\gamma}^{j}}\right\|_{l^{2}(\tau)}^{2}$.

We shall make repeated use of the following inequality:

Lemma 1 For all $m \leq 5$,

$$
\left\|\sum_{k=0}^{\frac{j}{2}}\left(\begin{array}{l}
j \\
k
\end{array}\right) a_{k+m} b_{j-k}\right\|_{l^{2}(\tau)} \leq C_{\tau}\left\|a_{j}\right\|_{l^{2}(\tau)}\left\|b_{j}\right\|_{l^{2}(\tau)}
$$

and symmetrically,

$$
\left\|\sum_{k=\frac{j}{2}}^{j}\left(\begin{array}{l}
j \\
k
\end{array}\right) a_{k} b_{j-k+m}\right\|_{l^{2}(\tau)} \leq C_{\tau}\left\|a_{j}\right\|_{l^{2}(\tau)}\left\|b_{j}\right\|_{l^{2}(\tau)}
$$

Remark 1 In this lemma and in all the text, the notation $\frac{j}{2}$ that appears as an index in the sums is slightly abusive: it stands for the integer part of $\frac{j}{2}$.

Proof: Denoting $\alpha_{j}(\tau):=\tau^{j}(j !)^{-7 / 4}(j+1)^{10}$, we find

$$
\begin{aligned}
\left\|\sum_{k=0}^{\frac{j}{2}}\left(\begin{array}{l}
j \\
k
\end{array}\right) a_{k+m} b_{j-k}\right\|_{l^{2}(\tau)} & \lesssim\left\|\sum_{k=0}^{\frac{j}{2}}\left(\begin{array}{l}
j \\
k
\end{array}\right)^{-3 / 4}\left(\frac{(k+m) !}{k !}\right)^{7 / 4}(k+m+1)^{-10} \alpha_{k+m}(\tau) a_{k+m} \alpha_{j-k}(\tau) b_{j-k}\right\|_{l^{2}} \\
& \lesssim\left\|\sum_{k=0}^{+\infty}(k+1)^{\frac{7}{4} m-10} \alpha_{k+m}(\tau) a_{k+m} \alpha_{j-k}(\tau) b_{j-k}\right\|_{l^{2}}
\end{aligned}
$$

By a standard convolution inequality for discrete sums, we get

$$
\left\|\sum_{k=0}^{\frac{j}{2}}\left(\begin{array}{l}
j \\
k
\end{array}\right) a_{k+m} b_{j-k}\right\|_{l^{2}(\tau)} \leq\left\|(j+1)^{\frac{7}{4} m-10}\left(\alpha_{j}(\tau) a_{j}\right)\right\|_{l^{1}}\left\|\alpha_{j}(\tau) b_{j}\right\|_{l^{2}}
$$

As $m \leq 5$, we can use Cauchy Schwartz inequality to bound the first factor, which yields the result.

Let us also emphasize in a lemma some important relations between our energy functionals: 


\section{Lemma 2}

$$
E_{\omega}(t, \tau)-\dot{E}_{\omega}(t, \tau) \lesssim E_{g}^{1}(t, \tau)+E_{h}(t, \tau) \lesssim E_{\omega}(t, \tau)-\dot{E}_{\omega}(t, \tau)
$$

and

$$
\partial_{\tau} E_{\omega}(t, \tau)-\partial_{\tau} \dot{E}_{\omega}(t, \tau) \lesssim \partial_{\tau} E_{g}^{1}(t, \tau)+\partial_{\tau} E_{h}(t, \tau) \lesssim \partial_{\tau} E_{\omega}(t, \tau)-\partial_{\tau} \dot{E}_{\omega}(t, \tau) .
$$

These relations, to be proved in the appendix, follow from a direct but crucial representation of $\partial_{x}^{j} u$ in terms of $g_{j}$ (see (2.12) for the definition of $g_{j}$ ):

Lemma 3 One can write:

$$
\partial_{x}^{j} u(t, x, y)=\left\{\begin{array}{l}
\omega(t, x, y) \int_{3}^{y}\left(\psi+\frac{(1-\psi)}{\omega}\right)^{-1} \frac{g_{j}}{\omega^{2}}+C_{j}(t, x) \omega(t, x, y), \quad y>a(t, x), \\
\omega(t, x, y) \int_{0}^{y}\left(\psi+\frac{(1-\psi)}{\omega}\right)^{-1} \frac{g_{j}}{\omega^{2}}, \quad y<a(t, x)
\end{array}\right.
$$

with $C_{j}:=-\partial_{x}^{j} u(t, x, 3) / \omega(t, x, 3)$.

Proof: The proof is trivial: integrate the relation

$$
\partial_{y} \frac{\partial_{x}^{j} u}{\omega}=\left(\psi+\frac{(1-\psi)}{\omega}\right)^{-1} \frac{g_{j}}{\omega^{2}}
$$

from 0 to $y$ and from 3 to $y$ respectively. We point out that the choice 3 is arbitrary.

We stress again that $u$ is assumed to be a smooth solution of the Prandtl equation, notably smooth across the critical curve $y=a(t, x)$. Note also that (3.6) formally yields the relation

$$
\partial_{x}^{j} u(t, x, y)=\omega(t, x, y) \int_{0}^{y}\left(\psi+\frac{(1-\psi)}{\omega}\right)^{-1} \frac{g_{j}}{\omega^{2}}
$$

for all $y$. However, the right-hand side does not make sense for $y>a(t, x)$ : as $\omega$ degenerates near $y=a$, the integral is not properly defined. One can not use it to bound nicely $\partial_{x}^{j} u$ in terms of $g_{j}$, even away from $y=a$. This is why we substitute to this formula the decomposition

$$
\partial_{x}^{j} u(t, x, y)=\omega(t, x, y) \int_{3}^{y}\left(\psi+\frac{(1-\psi)}{\omega}\right)^{-1} \frac{g_{j}}{\omega^{2}}+C_{j}(t, x) \omega(t, x, y)
$$

with a first term that depends nicely on $g_{j}$ away from $y=a$, and a second term that, broadly speaking, will be controlled by the hydrostatic energy.

Let us conclude this paragraph by a trivial remark: for any energy functional $E=E_{\omega}, E_{h} \ldots$ that will be used in the text, one has $E \leq \partial_{\tau} E$. This will be used many times without mentioning. 


\subsection{Estimate of the vorticity energy}

We focus here on the energy $\dot{E}_{\omega}(t, \tau)$ defined in (2.8). We also introduce the functional

$$
\dot{D}_{\omega}(t, \tau):=\sum_{j=0}^{+\infty}\left(\tau^{j}(j !)^{-7 / 4}(j+1)^{10}\right)^{2}\left\|\partial_{y} \omega(t, \cdot)\right\|_{\dot{\mathcal{H}}_{\gamma}^{j}}^{2} .
$$

which will appear due to to the viscous term in the Prandtl equation. We shall establish

\section{Proposition 1 (Estimate on the vorticity energy)}

Let $T>0, \tau \geq 1, s, \gamma, \sigma$ be as in Theorem 1. Let $u$ be a smooth solution of the Prandtl equation over $] 0, T]$. Assume that the vorticity $\omega$ satisfies (3.1) over ]0,T], and that

$$
\left.\left.E_{\omega}(t, \tau) \leq M, \quad M>0, \quad \forall t \in\right] 0, T\right]
$$

Then, one has for some $C>0$ and all $t \in] 0, T]$ :

$$
\partial_{t} \dot{E}_{\omega}(t, \tau)+\dot{D}_{\omega}(t, \tau) \leq C \partial_{\tau} E_{\omega}(t, \tau)
$$

The starting point is to write down an equation on $\omega_{J}:=\partial^{J} \omega, J=\left(j_{1}, j_{2}\right) \in \mathbb{N}^{2}, \quad 0<j_{2} \leq s$. Differentiating the vorticity equation (2.2), we find

$$
\partial_{t} \omega_{J}+u \partial_{x} \omega_{J}+v \partial_{y} \omega_{J}-\partial_{y}^{2} \omega_{J}=-\left[\partial^{J}, u\right] \partial_{x} \omega-\left[\partial^{J}, v\right] \partial_{y} \omega .
$$

After multiplication by $(1+y)^{2 \gamma+2 j_{2}} \omega_{J}$, integration over $\mathbb{T} \times \mathbb{R}_{+}$and standard integration by parts, we get

$$
\begin{aligned}
& \frac{1}{2} \frac{d}{d t}\left\|(1+y)^{\gamma+j_{2}} \omega_{J}(t, \cdot)\right\|_{L^{2}\left(\mathbb{T} \times \mathbb{R}_{+}\right)}^{2}+\left\|(1+y)^{\gamma+j_{2}} \partial_{y} \omega_{J}(t, \cdot)\right\|_{L^{2}\left(\mathbb{T} \times \mathbb{R}_{+}\right)}^{2} \\
= & -\int_{\mathbb{T} \times \mathbb{R}_{+}}(1+y)^{2 \gamma+2 j_{2}}\left[\partial^{J}, u\right] \partial_{x} \omega \omega_{J}-\int_{\mathbb{T} \times \mathbb{R}_{+}}(1+y)^{2 \gamma+2 j_{2}}\left[\partial^{J}, v\right] \partial_{y} \omega \omega_{J} \\
& -\left(2 \gamma+2 j_{2}\right) \int_{\mathbb{T} \times \mathbb{R}_{+}}(1+y)^{2 \gamma+2 j_{2}-1}\left(\partial_{y} \omega_{J}-v \omega_{J}\right) \omega_{J}+\int_{\mathbb{T} \times\{0\}} \partial_{y} \omega_{J} \omega_{J} .
\end{aligned}
$$

Multiplying by $\left(\tau^{|J|}(|J| !)^{-7 / 4}|J|^{10}\right)^{2}$ and summing over $J \in \mathbb{N} \times[|1, s|]$, we obtain

$$
\begin{aligned}
\partial_{t} \dot{E}_{\omega}(t, \tau) & +\dot{D}_{\omega}(t, \tau) \\
& \lesssim\left(\left\|A_{j}\right\|_{l^{2}(\tau)}+\left\|B_{j}\right\|_{l^{2}(\tau)}+\left\|C_{j}\right\|_{l^{2}(\tau)}+\left\|D_{j}\right\|_{l^{2}(\tau)}\right)\left\|j^{1 / 2}\right\| \omega\left\|_{\dot{\mathcal{H}}_{\gamma}^{j}}\right\|_{l^{2}(\tau)}+\left\|E_{j}\right\|_{l^{1}(\tau)} \\
& \lesssim\left(\left\|A_{j}\right\|_{l^{2}(\tau)}+\left\|B_{j}\right\|_{l^{2}(\tau)}+\left\|C_{j}\right\|_{l^{2}(\tau)}+\left\|D_{j}\right\|_{l^{2}(\tau)}\right) \sqrt{\partial_{\tau} \dot{E}_{\omega}(t, \tau)}+\left\|E_{j}\right\|_{l^{1}(\tau)}
\end{aligned}
$$


where

$$
\begin{aligned}
& A_{j}^{2}:=\frac{1}{j} \sum_{\substack{J=\left(j_{1}, j_{2}\right) \in \mathbb{N}^{2} \\
|J|=j, 0<j_{2} \leq s}}\left\|(1+y)^{\gamma+j_{2}}\left[\partial^{J}, u\right] \partial_{x} \omega\right\|_{L^{2}\left(\mathbb{T} \times \mathbb{R}_{+}\right)}^{2}, \\
& B_{j}^{2}:=\frac{1}{j} \sum_{\substack{J=\left(j_{1}, j_{2}\right) \in \mathbb{N}^{2} \\
|J|=j, 0<j_{2} \leq s}}\left\|(1+y)^{\gamma+j_{2}}\left[\partial^{J}, v\right] \partial_{y} \omega\right\|_{L^{2}\left(\mathbb{T} \times \mathbb{R}_{+}\right)}^{2}, \\
& C_{j}^{2}:=\frac{1}{j} \sum_{\substack{J=\left(j_{1}, j_{2}\right) \in \mathbb{N}^{2} \\
|J|=j, 0<j_{2} \leq s}}\left\|(1+y)^{\gamma+j_{2}-1} v \omega_{J}\right\|_{L^{2}\left(\mathbb{T} \times \mathbb{R}_{+}\right)}^{2}, \\
& D_{j}^{2}:=\frac{1}{j} \sum_{\substack{J=\left(j_{1}, j_{2}\right) \in \mathbb{N}^{2} \\
|J|=j, 0<j_{2} \leq s}}\left\|(1+y)^{\gamma+j_{2}-1} \partial_{y} \omega_{J}\right\|_{L^{2}\left(\mathbb{T} \times \mathbb{R}_{+}\right)}^{2}, \\
& E_{j}=\sum_{\substack{J=\left(j_{1}, j_{2}\right) \in \mathbb{N}^{2} \\
|J|=j, 0<j_{2} \leq s}} \int_{\mathbb{T} \times\{0\}} \partial_{y} \omega_{J} \omega_{J} .
\end{aligned}
$$

Note that the first term at the right-hand side. comes from Cauchy-Schwarz inequality, first in $L^{2}\left(\mathbb{T} \times \mathbb{R}_{+}\right)$, then in $l^{2}(\mathbb{N} \times[|1, s|])$.

\section{Estimate on $A_{j}$}

We first write, for $J \in \mathbb{N} \times[|1, s|],|J|=j$ :

$$
(1+y)^{\gamma+j_{2}}\left[\partial^{J}, u\right] \partial_{x} \omega=\sum_{\substack{K \leq J \\
|K|>0}}\left(\begin{array}{c}
J \\
K
\end{array}\right)(1+y)^{k_{2}} \partial^{K} u(1+y)^{\gamma+j_{2}-k_{2}} \partial^{J-K} \partial_{x} \omega .
$$

As $\gamma \geq 1$, we obtain

$$
(1+y)^{\gamma+j_{2}}\left|\left[\partial^{J}, u\right] \partial_{x} \omega\right| \leq \sum_{\substack{K \leq J \\
|K|>0}}\left(\begin{array}{c}
J \\
K
\end{array}\right)\left|(1+y)^{\gamma+k_{2}-1} \partial^{K} u\right|\left|(1+y)^{\gamma+j_{2}-k_{2}} \partial^{J-K} \partial_{x} \omega\right| .
$$

Then, we write

$$
\begin{aligned}
& \left\|(1+y)^{\gamma+j_{2}}\left[\partial^{J}, u\right] \partial_{x} \omega\right\|_{L^{2}\left(\mathbb{T} \times \mathbb{R}_{+}\right)} \\
& \leq \sum_{\substack{K \leq J \\
\frac{|J|}{2} \geq|K|>0}}\left(\begin{array}{c}
J \\
K
\end{array}\right) a_{K}^{1} b_{J-K}^{1}+\sum_{\substack{K \leq J \\
J \geq|K|>\frac{|J|}{2}}}\left(\begin{array}{c}
J \\
K
\end{array}\right) a_{K}^{2} b_{J-K}^{2}
\end{aligned}
$$

where

$$
\begin{array}{ll}
a_{K}^{1}:=\left\|(1+y)^{\gamma+k_{2}-1} \partial^{K} u\right\|_{L^{\infty}\left(\mathbb{T} \times \mathbb{R}_{+}\right)}, & b_{J-K}^{1}:=\left\|(1+y)^{\gamma+j_{2}-k_{2}} \partial^{J-K} \partial_{x} \omega\right\|_{L^{2}\left(\mathbb{T} \times \mathbb{R}_{+}\right)} \\
a_{K}^{2}:=\left\|(1+y)^{\gamma+k_{2}-1} \partial^{K} u\right\|_{L^{2}\left(\mathbb{T} \times \mathbb{R}_{+}\right)}, & b_{J-K}^{2}:=\left\|(1+y)^{\gamma+j_{2}-k_{2}} \partial^{J-K} \partial_{x} \omega\right\|_{L^{\infty}\left(\mathbb{T} \times \mathbb{R}_{+}\right)}
\end{array}
$$

Treatment of the first sum. 
We first notice that $\left(\begin{array}{c}J \\ K\end{array}\right) \lesssim\left(\begin{array}{c}|J| \\ |K|\end{array}\right)$, uniformly for $J \in \mathbb{N} \times[|1, s|]$. Then, using Sobolev and Hardy inequalities (A.1), (A.2), we obtain easily that

$$
a_{K}^{1} \lesssim\|\omega\|_{\mathcal{H}_{\gamma}^{|K|}}+\|\omega\|_{\mathcal{H}_{\gamma}^{|K|+1}}
$$

Also, clearly

$$
b_{J-K}^{1} \lesssim\|\omega\|_{\mathcal{H}_{\gamma}^{|J-K|+1}} .
$$

Hence, we obtain $(k=|K|, j=|J|)$ :

$$
\begin{aligned}
& \frac{1}{j^{1 / 2}} \sum_{\substack{K \leq J \\
\frac{J J}{2} \geq|K|>0}}\left(\begin{array}{c}
J \\
K
\end{array}\right) a_{K}^{1} b_{J-K}^{1} \lesssim \frac{1}{j^{1 / 2}} \sum_{k=1}^{\frac{j}{2}}\left(\begin{array}{c}
j \\
k
\end{array}\right)\|\omega\|_{\mathcal{H}_{\gamma}^{k}}\|\omega\|_{\mathcal{H}_{\gamma}^{j-k+1}}+\frac{1}{j^{1 / 2}} \sum_{k=1}^{\frac{j}{2}}\left(\begin{array}{c}
j \\
k
\end{array}\right)\|\omega\|_{\mathcal{H}_{\gamma}^{k+1}}\|\omega\|_{\mathcal{H}_{\gamma}^{j-k+1}} \\
& \lesssim \frac{1}{j^{1 / 2}} \sum_{k=0}^{\frac{j}{2}-1}\left(\begin{array}{c}
j \\
k+1
\end{array}\right)\|\omega\|_{\mathcal{H}_{\gamma}^{k+1}}\|\omega\|_{\mathcal{H}_{\gamma}^{j-k}}+\frac{1}{j^{1 / 2}} \sum_{k=0}^{\frac{j}{2}-1}\left(\begin{array}{c}
j \\
k+1
\end{array}\right)\|\omega\|_{\mathcal{H}_{\gamma}^{k+2}}\|\omega\|_{\mathcal{H}_{\gamma}^{j-k}} \\
& \lesssim \sum_{k=0}^{\frac{j}{2}}\left(\begin{array}{c}
j \\
k
\end{array}\right)\|\omega\|_{\mathcal{H}_{\gamma}^{k+1}}\left((j-k)^{1 / 2}\|\omega\|_{\mathcal{H}_{\gamma}^{j-k}}\right)+\sum_{k=0}^{\frac{j}{2}}\left(\begin{array}{l}
j \\
k
\end{array}\right)\|\omega\|_{\mathcal{H}_{\gamma}^{k+2}}\left((j-k)^{1 / 2}\|\omega\|_{\mathcal{H}_{\gamma}^{j-k}}\right) .
\end{aligned}
$$

We finally control the $l^{2}(\tau)$ norm of the right-hand side using Lemma 1 (we take $m=1$ for the first term, $m=2$ for the second term). We end up with

$$
\left\|\frac{1}{j^{1 / 2}} \sum_{\substack{J=\left(j_{1}, j_{2}\right) \in \mathbb{N}^{2} \\
|J|=j, 0<j_{2} \leq s}} \sum_{\substack{|J| \\
\frac{|J|}{2} \geq|K|>0}}\left(\begin{array}{c}
J \\
K
\end{array}\right) a_{K}^{1} b_{J-K}^{1}\right\|_{l^{2}(\tau)} \lesssim \sqrt{E_{\omega}(t, \tau)} \sqrt{\partial_{\tau} E_{\omega}(t, \tau)} \lesssim \sqrt{\partial_{\tau} E_{\omega}(t, \tau)}
$$

Treatment of the second sum.

We proceed as for the first sum, reversing the role of factors $a$ and $b$ : more precisely, we apply the Hardy inequality (A.2) to $a_{K}^{2}$ :

$$
a_{K}^{2} \lesssim\|\omega\|_{\mathcal{H}_{\gamma}^{|K|}}
$$

and the Sobolev bound (A.1) to $b_{J-K}^{2}$. Note that, due to the last two terms at the right-hand side of (A.1), the quantities $\partial_{y} \partial^{J-K} \partial_{x} \omega$ and $\partial_{y} \partial^{J-K} \partial_{x}^{2} \omega$ are needed to control $b_{J-K}^{2}$. In the special case where $j_{2}=s, k_{2}=0$, they involve $s+1 \quad \partial_{y}$-derivatives. Thus, they can not be controlled by $\|\omega\|_{\mathcal{H}_{\gamma}^{|J-K|+m}}$, similarly to what we did for $a_{K}^{1}$ (the definition of $\mathcal{H}_{\gamma}^{j}$ spaces involves only $j_{2} \leq s \quad \partial_{y}$-derivatives). Hence, in this special case, we rather use the inequality

$$
\left\|(1+y)^{\gamma+j_{2}-k_{2}} \partial_{y} \partial^{J-K} \partial_{x}^{m} \omega\right\|_{L^{2}} \lesssim\left\|\partial_{y} \omega\right\|_{\dot{\mathcal{H}}^{|J-K|+m}}, \quad m=1,2 .
$$

We insist that the r.h.s involves the homogeneous space $\dot{\mathcal{H}}^{|J-K|+m}$, because $j_{2}-k_{2} \neq 0$ $\left(j_{2}=s, k_{2}=0\right)$. This is important to use the dissipation term $\dot{D}_{\omega}$. Eventually, we find

$$
b_{J-K}^{2} \lesssim \sum_{m=1}^{3}\|\omega\|_{\mathcal{H}_{\gamma}^{|J-K|+m}}+\left\|\partial_{y} \omega\right\|_{\dot{\mathcal{H}}_{\gamma}^{|J-K|+m}}
$$


Then,

$$
\frac{1}{j^{1 / 2}} \sum_{\substack{K \leq J \\
J \geq|K|>\frac{|J|}{2}}}\left(\begin{array}{c}
J \\
K
\end{array}\right) a_{K}^{2} b_{J-K}^{2} \leq \frac{1}{j^{1 / 2}} \sum_{m=1}^{3} \sum_{k=\frac{j}{2}}^{j}\left(\begin{array}{l}
j \\
k
\end{array}\right)\|\omega\|_{\mathcal{H}_{\gamma}^{k}}\left(\|\omega\|_{\mathcal{H}_{\gamma}^{j-k+m}}+\left\|\partial_{y} \omega\right\|_{\dot{\mathcal{H}}_{\gamma}^{j-k+m}}\right)
$$

We crudely bound $j^{-1 / 2}$ by 1 , and apply Lemma 1, to find

$$
\begin{aligned}
\left\|\frac{1}{j^{1 / 2}} \sum_{\substack{J=\left(j_{1}, j_{2}\right) \in \mathbb{N}^{2} \\
|J|=j, 0<j_{2} \leq s}} \sum_{\substack{K \leq J \\
J \geq|K|>\frac{|J|}{2}}}\left(\begin{array}{c}
J \\
K
\end{array}\right) a_{K}^{2} b_{J-K}^{2}\right\|_{l^{2}(\tau)} & \lesssim \sqrt{E_{\omega}(t, \tau)}\left(\sqrt{E_{\omega}(t, \tau)}+\sqrt{\dot{D}_{\omega}(t, \tau)}\right) \\
& \lesssim \sqrt{E_{\omega}(t, \tau)}+\sqrt{\dot{D}_{\omega}(t, \tau)}
\end{aligned}
$$

Gathering of the bounds (3.14)-(3.15) leaves us with

$$
\left\|A_{j}\right\|_{l^{2}(\tau)} \lesssim \sqrt{E_{\omega}(t, \tau)}+\sqrt{\partial_{\tau} E_{\omega}(t, \tau)}+\sqrt{\dot{D}_{\omega}(t, \tau)} \lesssim \sqrt{\partial_{\tau} E_{\omega}(t, \tau)}+\sqrt{\dot{D}_{\omega}(t, \tau)}
$$

\section{Estimate on $B_{j}$}

Let us first point out that $v=-\int_{0}^{y} \partial_{x} u$ does not decay at $y=\infty$. One has

$$
\begin{aligned}
\left\|\partial_{x}^{k} v\right\|_{L^{\infty}} & \lesssim\left\|\int_{0}^{+\infty}\left|\partial_{x}^{k+1} u\right| d y\right\|_{L^{\infty}(\mathbb{T})} \\
& \lesssim\left\|\partial_{x}^{k+1} u\right\|_{L^{\infty}\left(L_{\gamma-1}^{2}\right)} \lesssim\left\|\partial_{x}^{k+1} u\right\|_{L^{2}\left(L_{\gamma-1}^{2}\right)}+\left\|\partial_{x}^{k+2} u\right\|_{L^{2}\left(L_{\gamma-1}^{2}\right)} \\
& \lesssim\left\|\partial_{x}^{k+1} \omega\right\|_{L^{2}\left(L_{\gamma}^{2}\right)}+\left\|\partial_{x}^{k+2} \omega\right\|_{L^{2}\left(L_{\gamma}^{2}\right)}
\end{aligned}
$$

where the last line comes from (A.3). For $K=\left(k_{1}, k_{2}\right)$ with $k_{2}>0$, we find

$$
\left\|(1+y)^{\gamma+k_{2}-1} \partial^{K} v\right\|_{L^{\infty}}=\left\|(1+y)^{\gamma+k_{2}-1} \partial_{x}^{k_{1}+1} \partial_{y}^{k_{2}-1} u\right\|_{L^{\infty}}
$$

which, as seen before, can be controlled through (A.1) and (A.2).

Proceeding (almost) as for $A_{j}$, we get

$$
\begin{aligned}
& \left\|(1+y)^{\gamma+j_{2}}\left[\partial^{J}, v\right] \partial_{y} \omega\right\|_{L^{2}\left(\mathbb{T} \times \mathbb{R}_{+}\right)} \\
& \leq \sum_{\substack{\left|K \leq J \\
\frac{|J|}{2} \geq\right| K \mid>0}}\left(\begin{array}{c}
J \\
K
\end{array}\right) a_{K}^{1} b_{J-K}^{1}+\sum_{\substack{K \leq J, j_{2}-k_{2} \leq s-2 \\
J \geq|K|>\frac{|J|}{2}}}\left(\begin{array}{c}
J \\
K
\end{array}\right) a_{K}^{2} b_{J-K}^{2}+\sum_{\substack{K \leq J, s-1 \leq j_{2}-k_{2} \leq s \\
J \geq|K|>\frac{|J|}{2}}}\left(\begin{array}{c}
J \\
K
\end{array}\right) a_{K}^{3} b_{J-K}^{3} .
\end{aligned}
$$

where this time

$$
\begin{aligned}
& a_{K}^{1}:=\left\|(1+y)^{k_{2}} \partial^{K} v\right\|_{L^{\infty}\left(\mathbb{T} \times \mathbb{R}_{+}\right)}, \quad b_{J-K}^{1}:=\left\|(1+y)^{\gamma+j_{2}-k_{2}} \partial^{J-K} \partial_{y} \omega\right\|_{L^{2}\left(\mathbb{T} \times \mathbb{R}_{+}\right)} \\
& a_{K}^{2}:=\left\|(1+y)^{k_{2}-1} \partial^{K} v\right\|_{L^{2}\left(\mathbb{T} \times \mathbb{R}_{+}\right)}, b_{J-K}^{2}:=\left\|(1+y)^{\gamma+j_{2}-k_{2}+1} \partial^{J-K} \partial_{y} \omega\right\|_{L^{\infty}\left(\mathbb{T} \times \mathbb{R}_{+}\right)}, \\
& a_{K}^{3}:=\left\|(1+y)^{k_{2}} \partial^{K} v\right\|_{L^{\infty}\left(\mathbb{T} \times \mathbb{R}_{+}\right)}, \quad b_{J-K}^{3}:=\left\|(1+y)^{\gamma+j_{2}-k_{2}} \partial^{J-K} \partial_{y} \omega\right\|_{L^{2}\left(\mathbb{T} \times \mathbb{R}_{+}\right)} .
\end{aligned}
$$

Treatment of the first sum 
Thanks to (3.17) and (3.18), we find

$$
a_{K}^{1} \lesssim \sum_{m=1}^{2}\|\omega\|_{\mathcal{H}_{\gamma}^{|K|+m}}
$$

As regards $b_{K}^{1}$, one must take care of the special case: $j_{2}=s, k_{2}=0$, for which $\partial^{J-K} \partial_{y} \omega$ involves $s+1$ derivatives with respect to $y$. Hence, we write

$$
\left\|b_{K}^{1}\right\| \leq\|\omega\|_{\mathcal{H}_{\gamma}^{|J-K|+1}}+\left\|\partial_{y} \omega\right\|_{\dot{\mathcal{H}}_{\gamma}^{|J-K|}}
$$

where the last term at the right-hand side accounts for the special case. Proceeding as for $A_{j}$, relying on Lemma 1, we find

$$
\left\|\frac{1}{j^{1 / 2}} \sum_{\substack{J=\left(j_{1}, j_{2}\right) \in \mathbb{N}^{2} \\
|J|=j, 0<j_{2} \leq s}} \sum_{\substack{K \leq J \\
\frac{|J|}{2} \geq|K|>0}}\left(\begin{array}{c}
J \\
K
\end{array}\right) a_{K}^{1} b_{J-K}^{1}\right\|_{l^{2}(\tau)} \lesssim \sqrt{\partial_{\tau} E_{\omega}(t, \tau)}+\sqrt{\dot{D}_{\omega}(t, \tau)} .
$$

\section{Treatment of the second sum}

We handle the second sum as we did for $A_{j}$ : we apply Hardy inequality to $a_{K}^{2}$, Sobolev inequality to $b_{K}^{2}$. Remark that we put factor $(1+y)^{k_{2}-1}$ in front of $\partial^{K} v$, in the definition of $a_{K}^{2}$. It allows the $L^{2}$ norm to be finite even in the case $k_{2}=0$. Of course, this forces a factor $(1+y)^{\gamma+j_{2}-k_{2}+1}$ in front of $\partial^{J-K} \partial_{y} \omega$, which is harmless thanks to the extra $\partial_{y}$.

Moreover, as we restrict here to indices satisfying $j_{2}-k_{2} \leq s-2$, the control of $b_{K}^{2}$ by the inequality (A.1) (which involves for instance $\partial^{J-K} \partial_{y}^{2} \omega$ ) only requires terms with less than $s$ derivatives in $y$. We leave the details to the reader. We get

$$
\left\|\frac{1}{j^{1 / 2}} \sum_{\substack{J=\left(j_{1}, j_{2}\right) \in \mathbb{N}^{2} \\
|J|=j, 0<j_{2} \leq s}} \sum_{\substack{K \leq J, j_{2}-k_{2} \leq s-2 \\
J \geq|K|>\frac{|J|}{2}}}\left(\begin{array}{c}
J \\
K
\end{array}\right) a_{K}^{2} b_{J-K}^{2}\right\|_{l^{2}(\tau)} \lesssim E_{\omega}(t, \tau) \lesssim \sqrt{E_{\omega}(t, \tau)}
$$

Treatment of the third sum

Note that the third sum is empty except when $j_{2}=s$ (in which case $k_{2} \in\{0,1\}$ ) or when $j_{2}=s-1$ (in which case $k_{2}=0$ ). In both cases, the important thing to notice is that

$$
|K| \leq k_{1}+1 \leq j_{1}-1 \leq|J|-s, \quad \text { and }\left(\begin{array}{c}
J \\
K
\end{array}\right) \leq\left(\begin{array}{c}
|J| \\
|K|+m
\end{array}\right) \forall m \leq s
$$

Using once again (3.17), (3.18) and (A.1), we end up with

$$
a_{K}^{3} \lesssim \sum_{m=1}^{2}\|\omega\|_{\mathcal{H}_{\gamma}^{|K|+m}}
$$

whereas

$$
b_{K}^{3} \lesssim\left\|\partial_{y} \omega\right\|_{\dot{\mathcal{H}}_{\gamma}^{|J-K|}}
$$


We get, taking (3.22) into account:

$$
\frac{1}{j^{1 / 2}} \sum_{\substack{K \leq J, s-1 \leq j_{2}-k_{2} \leq s \\
J \geq|K|>\frac{|J|}{2}}}\left(\begin{array}{c}
J \\
K
\end{array}\right) a_{K}^{3} b_{J-K}^{3} \lesssim \frac{1}{j^{1 / 2}} \sum_{m=1}^{2} \sum_{k=\frac{j}{2}}^{j-s}\left(\begin{array}{c}
j \\
k+m
\end{array}\right)\|\omega\|_{\mathcal{H}_{\gamma}^{k+m}}\left\|\partial_{y} \omega\right\|_{\dot{\mathcal{H}}_{\gamma}^{j-k}}
$$

We can bound $\frac{1}{j^{1 / 2}}$ by 1 , and make a change of index: $k^{\prime}=k+m$. Then, application of Lemma 1 leads to

$$
\left\|\frac{1}{j^{1 / 2}} \sum_{\substack{K \leq J, s-1 \leq j_{2}-k_{2} \leq s \\
J \geq|K|>\frac{|J|}{2}}}\left(\begin{array}{c}
J \\
K
\end{array}\right) a_{K}^{3} b_{J-K}^{3}\right\|_{l^{2}(\tau)} \lesssim \sqrt{E_{\omega}(t, \tau)} \sqrt{\dot{D}_{\omega}(t, \tau)} \lesssim \sqrt{\dot{D}_{\omega}(t, \tau)}
$$

Gathering of the bounds (3.201)-(3.21)-(3.23) leads to

$$
\left\|B_{j}\right\|_{l^{2}(\tau)} \lesssim \sqrt{E_{\omega}(t, \tau)}+\sqrt{\partial_{\tau} E_{\omega}(t, \tau)}+\sqrt{\dot{D}_{\omega}(t, \tau)} \lesssim \sqrt{\partial_{\tau} E_{\omega}(t, \tau)}+\sqrt{\dot{D}_{\omega}(t, \tau)}
$$

\section{Estimate on $C_{j}$}

Clearly,

$$
\left|C_{j}\right| \leq\left\|\frac{v}{(1+y)}\right\|\left\|_{L^{\infty}}\right\| \omega\left\|_{\dot{\mathcal{H}}_{\gamma}^{j}} \lesssim \sqrt{E_{\omega}(t, \tau)}\right\| \omega \|_{\dot{\mathcal{H}}_{\gamma}^{j}}
$$

see (3.17). Thus,

$$
\left\|C_{j}\right\|_{l^{2}(\tau)} \lesssim \sqrt{E_{\omega}(t, \tau)} \sqrt{\dot{E}_{\omega}(t, \tau)} \lesssim \sqrt{E_{\omega}(t, \tau)}
$$

\section{Estimate on $D_{j}$}

Clearly,

$$
\left\|D_{j}\right\|_{l^{2}(\tau)} \lesssim \sqrt{\dot{D}_{\omega}(t, \tau)}
$$

\section{Estimate on $E_{j}$}

To handle the boundary term, a simple application of the trace theorem is not enough. One shall adapt ideas from [17]. The main point is to reduce the number of derivatives in the boundary term $\left.\partial_{y} \omega_{I} \omega_{I}\right|_{y=0}$, thanks to the equation. For instance, one can observe that

$$
\left.\partial_{y} \omega\right|_{y=0}=\left.\left(\partial_{t} u+u \partial_{x} u+v \partial_{y} u\right)\right|_{y=0}=0 .
$$

Then,

$$
\left.\partial_{y}^{3} \omega\right|_{y=0}=\left.\partial_{y}\left(\partial_{t} \omega+u \partial_{x} \omega+v \partial_{y} \omega\right)\right|_{y=0}=\left.\omega \partial_{x} \omega\right|_{y=0}
$$

For higher derivatives, one has

\section{Lemma 4 (from [17, Lemma 5.9])}

For $j_{2} \geq 4$ an even number, $\partial_{y} \omega_{J}$ is a linear combination of terms of the form

$$
\partial_{x}^{j_{1}}\left(\left.\prod_{l=1}^{N} \partial_{x}^{\alpha_{l}} \partial_{y}^{\beta_{l}} \omega\right|_{y=0}\right)
$$


with

$$
2 \leq N \leq \frac{j_{2}}{2}, \quad\left\{\begin{array}{l}
\sum_{l=1}^{N} 3 \alpha_{l}+\beta_{l}=j_{2}+1, \\
\sum_{l=1}^{N} \alpha_{l} \leq \frac{j_{2}}{2}-1, \quad \sum_{l=1}^{N} \beta_{l} \leq j_{2}-2, \\
\alpha_{l}+\beta_{l} \leq j_{2}-1, \quad \forall l=1 \ldots N .
\end{array}\right.
$$

Besides this lemma, we need a slight generalization of Lemma 1, whose proof is left to the reader:

Lemma 5 Let $N \geq 2, m_{2}, \ldots, m_{N} \leq 5$. Let

$$
K_{1}(j):=\left\{\left(k_{1}, \ldots, k_{N}\right) \in \mathbb{N}^{N}, \quad \text { s.t. } k_{1}+\cdots+k_{n}=j, \quad k_{1} \geq \frac{j}{n}\right\}
$$

for all $j \in \mathbb{N}$. Then, for sequences $a_{j}^{l}, j \in \mathbb{N}, l=1 \ldots N$, one has

$$
\left\|\sum_{\left(k_{1}, \ldots, k_{N}\right) \in K_{1}(j)} \frac{j !}{\prod_{l=1}^{N} k_{l} !} a_{k_{1}}^{1} \prod_{l=2}^{N} a_{k_{l}+m_{l}}^{l}\right\|_{l^{2}(\tau)} \lesssim \prod_{l=1}^{N}\left\|a_{j}^{l}\right\|_{l^{2}(\tau)} .
$$

We now write

$$
\begin{aligned}
E_{j}= & \sum_{\substack{J \in \mathbb{N}^{*} \times 2 \mathbb{N} \\
|J|=j, 0<j_{2} \leq s}} \int_{\mathbb{T} \times\{0\}} \partial_{y} \omega_{J} \omega_{J}+\sum_{\substack{J \in \mathbb{N}^{*} \times(2 \mathbb{N}+1) \\
|J|=j, 0<j_{2} \leq s}} \int_{\mathbb{T} \times\{0\}} \partial_{y} \omega_{J} \omega_{J} \\
& +\mathbf{1}_{[|1, \ldots, s|]}(j) \int_{\mathbb{T} \times\{0\}} \partial_{y}^{j+1} \omega \partial_{y}^{j} \omega:=E_{j}^{1}+E_{j}^{2}+E_{j}^{3}
\end{aligned}
$$

Study of $E_{j}^{1}$. Let $J \in \mathbb{N}^{*} \times 2 \mathbb{N},|J|=j, 0<j_{2} \leq s$. We write

$$
\begin{aligned}
\left|\int_{\mathbb{T} \times\{0\}} \partial_{y} \omega_{J} \omega_{J}\right| & \leq\left\|\left.\partial_{y} \omega_{J}\right|_{y=0}\right\|_{L^{2}(\mathbb{T})}\left\|\left.\omega_{J}\right|_{y=0}\right\|_{L^{2}(\mathbb{T})} \\
& \leq\left\|\left.\partial_{y} \omega_{J}\right|_{y=0}\right\|_{L^{2}(\mathbb{T})}\left\|\omega_{J}\right\|_{L^{2}\left(\mathbb{T} \times \mathbb{R}_{+}\right)}^{1 / 2}\left\|\partial_{y} \omega_{J}\right\|_{L^{2}\left(\mathbb{T} \times \mathbb{R}_{+}\right)}^{1 / 2} \leq\left\|\left.\partial_{y} \omega_{J}\right|_{y=0}\right\|_{L^{2}(\mathbb{T})}\left\|\partial_{y} \omega\right\|_{\mathcal{H}_{\gamma}^{j}}
\end{aligned}
$$

applying the trace theorem and then the Hardy inequality (A.2) to the last factor. As regards the first factor, one must use the reductions seen above. We focus on the case $j_{2} \geq 4$ (and even), which is treated thanks to Lemma 4. The case $j_{2}=2$, involving (3.27), is simpler. The $L^{2}$ norm of $\left\|\left.\partial_{y} \omega_{J}\right|_{y=0}\right\|_{L^{2}(\mathbb{T})}$ can be bounded by a finite number of terms of the type $\left\|\partial_{x}^{j_{1}}\left(\left.\prod_{l=1}^{N} \partial_{x}^{\alpha_{l}} \partial_{y}^{\beta_{l}} \omega\right|_{y=0}\right)\right\|_{L^{2}(\mathbb{T})}$, where $N$ and the $\left(\alpha_{l}, \beta_{l}\right)$ 's satisfy the conditions of the lemma.

As usual, we use the Lebnitz formula to write

$$
\begin{aligned}
\left\|\partial_{x}^{j_{1}}\left(\left.\prod_{l=1}^{N} \partial_{x}^{\alpha_{l}} \partial_{y}^{\beta_{l}} \omega\right|_{y=0}\right)\right\|_{L^{2}(\mathbb{T})}=\left\|\left.\sum_{k_{1}+\cdots+k_{n}=j_{1}} \frac{j_{1} !}{\prod_{l=1}^{N} k_{l} !} \prod_{l=1}^{N} \partial_{x}^{\alpha_{l}+k_{l}} \partial_{y}^{\beta_{l}} \omega\right|_{y=0}\right\|_{L^{2}(\mathbb{T})} \\
\leq \sum_{l^{\prime}=1}^{N}\left\|\left.\sum_{\left(k_{1}, \ldots, k_{n}\right) \in K_{l^{\prime}}\left(j_{1}\right)} \frac{j_{1} !}{\prod_{l=1}^{N} k_{l} !} \prod_{l=1}^{N} \partial_{x}^{\alpha_{l}+k_{l}} \partial_{y}^{\beta_{l}} \omega\right|_{y=0}\right\|_{L^{2}(\mathbb{T})}:=\sum_{l^{\prime}=1}^{N} \mathcal{N}_{l^{\prime}}
\end{aligned}
$$


where

$$
K_{l}\left(j_{1}\right):=\left\{\left(k_{1}, \ldots, k_{N}\right) \in \mathbb{N}^{N}, \quad \text { s.t. } \quad k_{1}+\cdots+k_{N}=j_{1}, \quad k_{l} \geq \frac{j_{1}}{N}\right\} .
$$

We now bound $\mathcal{N}_{1}$, the other terms being treated in the same way. We write

$$
\mathcal{N}_{1} \lesssim \sum_{\left(k_{1}, \ldots, k_{N}\right) \in K_{1}\left(j_{1}\right)} \frac{j_{1} !}{\prod_{l=1}^{N} k_{l} !}\left\|\left.\partial_{x}^{\alpha_{1}+k_{1}} \partial_{y}^{\beta_{1}} \omega\right|_{y=0}\right\|_{L^{2}(\mathbb{T})} \prod_{l=2}^{N}\left\|\partial_{x}^{\alpha_{l}+k_{l}} \partial_{y}^{\beta_{l}} \omega\right\|_{L^{\infty}\left(\mathbb{T} \times \mathbb{R}_{+}\right)}
$$

Note that

$$
\left\{\begin{array}{l}
\beta_{l}+2 \leq j_{2} \leq s \quad \text { for all } l, \\
\alpha_{1}+k_{1}+\beta_{1}+1 \leq j, \quad \alpha_{l}+k_{l}+\beta_{l}+2 \leq j, \quad l=2 \ldots N
\end{array}\right.
$$

$\left(k_{l} \leq j_{1}-1\right.$ for $l \geq 2$, as $\left.k_{1} \geq j_{1} / n\right)$. We use the the trace theorem with the first factor:

$$
\begin{aligned}
\left\|\left.\partial_{x}^{\alpha_{1}+k_{1}} \partial_{y}^{\beta_{1}} \omega\right|_{y=0}\right\|_{L^{2}(\mathbb{T})} & \lesssim\left\|\partial_{x}^{\alpha_{1}+k_{1}} \partial_{y}^{\beta_{1}} \omega\right\|_{L^{2}\left(\mathbb{T} \times \mathbb{R}_{+}\right)}^{1 / 2}\left\|\partial_{x}^{\alpha_{1}+k_{1}} \partial_{y}^{\beta_{1}+1} \omega\right\|_{L^{2}\left(\mathbb{T} \times \mathbb{R}_{+}\right)}^{1 / 2} \\
& \lesssim\left\|(1+y) \partial_{x}^{\alpha_{1}+k_{1}} \partial_{y}^{\beta_{1}+1} \omega\right\|_{L^{2}\left(\mathbb{T} \times \mathbb{R}_{+}\right)}
\end{aligned}
$$

The last bound comes from (A.2). Using the Sobolev imbedding (A.1) with the second factor. We get:

$$
\begin{aligned}
\mathcal{N}_{1} & \lesssim \sum_{\left(k_{1}, \ldots, k_{N}\right) \in K_{1}\left(j_{1}\right)} \frac{j_{1} !}{\prod_{l=1}^{N} k_{l} !}\|\omega\|_{\dot{\mathcal{H}}_{\gamma}^{\alpha_{1}+k_{1}+\beta_{1}+1}} \prod_{l=2}^{N} \sum_{m=0}^{2}\|\omega\|_{\dot{\mathcal{H}}_{\gamma}^{\alpha_{l}+k_{l}+\beta_{l}+m}} \\
& \lesssim \sum_{\left(k_{1}^{\prime}, \ldots, k_{N}^{\prime}\right) \in K_{1}\left(j^{\prime}\right)} \frac{j^{\prime} !}{\prod_{l=1}^{N} k_{l}^{\prime} !}\|\omega\|_{\dot{\mathcal{H}}_{\gamma}^{k_{1}^{\prime}}} \prod_{l=2}^{N} \sum_{m=0}^{2}\|\omega\|_{\dot{\mathcal{H}}_{\gamma}^{k_{l}^{\prime}+m}}
\end{aligned}
$$

The last inequality comes from the change of index

$$
k_{1}^{\prime}:=k_{1}+\alpha_{1}+\beta_{1}+1, \quad k_{l}^{\prime}:=k_{l}+\alpha_{l}+\beta_{l}+m, \quad l \geq 2
$$

noticing that $j^{\prime}:=j_{1}+\sum_{l=1}^{N}\left(\alpha_{l}+\beta_{l}\right)+1 \leq j$. By symmetry, the same bound applies to $\mathcal{N}_{2}, \mathcal{N}_{N}$. Finally, we deduce from Lemma 5 that

$$
\left\|E_{j}^{1}\right\|_{l^{1}(\tau)} \lesssim \sqrt{\dot{E}_{\omega}(t, \tau)} \sqrt{\dot{D}_{\omega}(t, \tau)} \lesssim \eta \dot{D}_{\omega}(t, \tau)+C_{\eta} \dot{E}_{\omega}(t, \tau)
$$

Study of $E_{j}^{2}$. Let $J \in \mathbb{N}^{*} \times(2 \mathbb{N}+1),|J|=j, 0<j_{2} \leq s$. Note that $j_{2} \leq s-1$ (because $s$ is even). We integrate by parts with respect to $x$ :

$$
\int_{\mathbb{T} \times\{0\}} \partial_{y} \omega_{J} \omega_{J}=-\int_{\mathbb{T} \times\{0\}} \partial_{x}^{\left(j_{1}-1\right)} \partial_{y}^{j_{2}+1} \omega \partial_{x}^{j_{1}+1} \partial_{y}^{j_{2}} \omega
$$

Then, we apply the boundary reduction lemma to the second factor in the integrand. From there, the treatment is exactly the same as in the first case, and leads to

$$
\left\|E_{j}^{2}\right\|_{l^{1}(\tau)} \lesssim \eta \dot{D}_{\omega}(t, \tau)+C_{\eta} \dot{E}_{\omega}(t, \tau)
$$


Study of $E_{j}^{3}$. Note that $E_{j}^{3}$ is non zero only if $j \leq s$. When $j=s$, one uses Lemma 4 . Otherwise,

$$
\sum_{j=0}^{s-1}\left|\int_{\mathbb{T} \times\{0\}} \partial_{y}^{j+1} \partial_{y}^{j} \omega\right| \leq \sum_{j=0}^{s-1}\left\|\partial_{y}^{j+1} \omega\right\|_{L^{2}\left(\mathbb{T} \times \mathbb{R}_{+}\right)}\left\|\partial_{y}^{j} \omega\right\|_{L^{2}\left(\mathbb{T} \times \mathbb{R}_{+}\right)}^{1 / 2}\left\|\partial_{y} \partial_{y}^{j+1} \omega\right\|_{L^{2}\left(\mathbb{T} \times \mathbb{R}_{+}\right)}^{1 / 2}
$$

We end up with

$$
\left\|E_{j}^{3}\right\|_{l^{1}(\tau)} \lesssim \sqrt{\dot{E}_{\omega}(t, \tau)} \sqrt{\dot{D}_{\omega}(t, \tau)} \lesssim \eta \dot{D}_{\omega}(t, \tau)+C_{\eta} \dot{E}_{\omega}(t, \tau) .
$$

Eventually,

$$
\left\|E_{j}\right\|_{l^{1}(\tau)} \lesssim \eta \dot{D}_{\omega}(t, \tau)+C_{\eta} \dot{E}_{\omega}(t, \tau) .
$$

Combining this last inequality with (3.16) $-(3.24)-(3.25)-(3.26)$, we obtain

$$
\begin{aligned}
\partial_{t} \dot{E}_{\omega}(t, \tau)+\dot{D}_{\omega}(t, \tau) & \lesssim\left(\sqrt{\partial_{\tau} E_{\omega}(t, \tau)}+\sqrt{\dot{D}_{\omega}(t, \tau)}\right) \sqrt{\dot{E}_{\omega}(t, \tau)}+\eta \dot{D}_{\omega}(t, \tau)+C_{\eta} \dot{E}_{\omega}(t, \tau) \\
& \lesssim \eta \dot{D}_{\omega}(t, \tau)+C_{\eta} \partial_{\tau} E_{\omega}(t, \tau)
\end{aligned}
$$

Taking $\eta$ small enough yields Proposition 1 .

\subsection{Estimate of the hydrostatic energy}

This section is devoted to the hydrostatic energy $E_{h}(t, \tau)$ defined in (2.9), with its "viscous" counterpart

$$
D_{h}(t, \tau):=\sum_{j=0}^{+\infty}\left(\tau^{j}(j !)^{-7 / 4}(j+1)^{10}\right)^{2}\left\|\partial_{y} h_{j}(t, \cdot)\right\|_{L^{2}\left(\mathbb{T} \times \mathbb{R}_{+}\right.}^{2} .
$$

\section{Proposition 2 (Estimate on the hydrostatic energy)}

Under the same assumptions as in Theorem Q 2 , one has for some $C>0$ and all $t \in] 0, T]$ :

$$
\partial_{t} E_{h}(t, \tau)+D_{h}(t, \tau) \leq C\left(\partial_{\tau} E_{\omega}(t, \tau)+\partial_{\tau} E_{g}^{2}(t, \tau)\right) .
$$

The first step is to establish the equation on the function $h_{j}$ defined by (2.10):

$$
h_{j}=\chi^{a} \frac{\partial_{x}^{j} \omega}{\sqrt{\partial_{y} \omega}}, \quad \chi^{a}(t, x, y)=\chi(y-a(t, x)) .
$$

Starting from the vorticity equation

$$
\partial_{t} \omega+u \partial_{x} \omega+v \partial_{y} \omega-\partial_{y}^{2} \omega=0,
$$

we get

$$
\begin{aligned}
\partial_{t} h_{j}+u \partial_{x} h_{j}+v \partial_{y}-\partial_{y}^{2} h_{j} & =-\frac{\chi^{a}}{\sqrt{\partial_{y} \omega}}\left[\partial_{x}^{j}, u\right] \partial_{x} \omega-\frac{\chi^{a}}{\sqrt{\partial_{y} \omega}}\left(\left[\partial_{x}^{j}, v\right] \partial_{y} \omega-\partial_{x}^{j} v \partial_{y} \omega\right) \\
& -\left[\frac{\chi^{a}}{\sqrt{\partial_{y} \omega}}, \partial_{t}+u \partial_{x}+v \partial_{y}-\partial_{y}^{2}\right] \partial_{x}^{j} \omega-\frac{\chi^{a}}{\sqrt{\partial_{y} \omega}} \partial_{x}^{j} v \partial_{y} \omega
\end{aligned}
$$


Note that we have singled out the term $\partial_{x}^{j} v \partial_{y} \omega=-\int_{y}^{0} \partial_{x}^{j+1} u \partial_{y} \omega$ in the commutator with $v \partial_{y}$. This is the hardest term to control, as it involves $j+1$ derivatives with respect to $x$. We shall use a cancellation property similar to (2.5). However, such cancellation will not be enough, and we shall rely on the extra regularity offered by the monotonicity energy $E_{g}^{2}$.

Performing a standard energy estimate on the previous equation, multypling by $\left(\tau^{j}(j !)^{-7 / 4}(j+1)^{10}\right)^{2}$, summing over $j$, we end up with

$\frac{1}{2} \partial_{t} E_{h}(t, \tau)+D_{h}(t, \tau) \leq\left(\left\|A_{j}\right\|_{l^{2}(\tau)}+\left\|B_{j}\right\|_{l^{2}(\tau)}+\left\|C_{j}\right\|_{l^{2}(\tau)}\right) \sqrt{\partial_{\tau} E_{h}(t, \tau)}+\left\|D_{j}\right\|_{l^{1}(\tau)}+\left\|E_{j}\right\|_{l^{1}(\tau)}$

where

$$
\begin{aligned}
A_{j} & :=\frac{1}{j^{1 / 2}}\left\|\frac{\chi^{a}}{\sqrt{\partial_{y} \omega}}\left[\partial_{x}^{j}, u\right] \partial_{x} \omega\right\|_{L^{2}}, \quad B_{j}:=\frac{1}{j^{1 / 2}}\left\|\frac{\chi^{a}}{\sqrt{\partial_{y} \omega}}\left(\left[\partial_{x}^{j}, v\right] \partial_{y} \omega-\partial_{x}^{j} v \partial_{y} \omega\right)\right\|_{L^{2}}, \\
C_{j} & :=\frac{1}{j^{1 / 2}}\left\|\left(\partial_{t}+u \partial_{x}+v \partial_{y}-\partial_{y}^{2}\right)\left(\frac{\chi^{a}}{\sqrt{\partial_{y} \omega}}\right) \partial_{x}^{j} \omega\right\|_{L^{2}}, \quad D_{j}:=2 \int_{\mathbb{T} \times \mathbb{R}_{+}} \partial_{y} \frac{\chi^{a}}{\sqrt{\partial_{y} \omega}} \partial_{y} \partial_{x}^{j} \omega h_{j} \\
E_{j} & :=\int_{\mathbb{T} \times \mathbb{R}_{+}} \frac{\chi^{a}}{\sqrt{\partial_{y} \omega}} \partial_{x}^{j} v \partial_{y} \omega h_{j} .
\end{aligned}
$$

Note that $h_{j}$ is compactly supported in $y$, so that no boundary integral is present on the right-hand side.

\section{Estimate on $A_{j}$}

We proceed here as for the term $A_{j}$ of subsection 3.2 . The treatment is actually simpler, as only $x$ derivatives of $u$ and $\omega$ are involved in the expression of $A_{j}$. By use of the Sobolev inequality (A.1), one never encounters more than one $y$-derivative, in particular never more than $s$. For brevity, we skip the details. We find

$$
\left\|A_{j}\right\|_{l^{2}(\tau)} \lesssim \sqrt{\partial_{\tau} E_{\omega}(t, \tau)}
$$

\section{Estimate on $B_{j}$}

Again, the treatment is parallel to the one of subsection 3.2. Denoting by $K^{a}$ the support of $\chi^{a}$, we find that

$$
\begin{aligned}
B_{j}^{1} & \lesssim \frac{1}{j^{1 / 2}} \sum_{k=1}^{j-1}\left(\begin{array}{l}
j \\
k
\end{array}\right)\left\|\partial_{x}^{k} v \partial_{y} \partial_{x}^{j-k} \omega\right\|_{L^{2}\left(\mathbb{T} \times K^{a}\right)} \\
& \lesssim \frac{1}{j^{1 / 2}} \sum_{k=1}^{\frac{j}{2}}\left(\begin{array}{l}
j \\
k
\end{array}\right)\left\|\partial_{x}^{k} v\right\|_{L^{\infty}\left(\mathbb{T} \times K^{a}\right)}\left\|\partial_{y} \partial_{x}^{j-k} \omega\right\|_{L^{2}\left(\mathbb{T} \times K^{a}\right)} \\
& +\frac{1}{j^{1 / 2}} \sum_{k=\frac{j}{2}}^{j-1}\left(\begin{array}{l}
j \\
k
\end{array}\right)\left\|\partial_{x}^{k} v\right\|_{L^{2}\left(\mathbb{T} \times K^{a}\right)}\left\|\partial_{y} \partial_{x}^{j-k} \omega\right\|_{L^{\infty}\left(\mathbb{T} \times K^{a}\right)}:=B_{j}^{1}+B_{j}^{2}
\end{aligned}
$$


For the first term, we set $k^{\prime}:=k-1$ and use (3.17) to get (we drop the prime)

$$
\begin{aligned}
B_{j}^{1} & \lesssim \frac{1}{j^{1 / 2}} \sum_{k=0}^{\frac{j}{2}-1}\left(\begin{array}{c}
j \\
k+1
\end{array}\right) \sum_{m=2}^{3}\|\omega\|_{\mathcal{H}_{\gamma}^{k+m}}\|\omega\|_{\mathcal{H}_{\gamma}^{j-k}} \\
& \lesssim \sum_{k=0}^{\frac{j}{2}} \sum_{m=2}^{3}\left(\begin{array}{l}
j \\
k
\end{array}\right)\|\omega\|_{\mathcal{H}_{\gamma}^{k+m}}\left((j-k)^{1 / 2}\|\omega\|_{\mathcal{H}_{\gamma}^{j-k}}\right)
\end{aligned}
$$

The second term $B_{j}^{2}$ is handled in a symmetric way, thanks to the fact that index $k$ stops at $j-1$. Applying Lemma 1, we eventually derive the bound:

$$
\left\|B_{j}\right\|_{l^{2}(\tau)} \lesssim \sqrt{E_{\omega}(t, \tau)} \sqrt{\partial_{\tau} E_{\omega}(t, \tau)} \lesssim \sqrt{\partial_{\tau} E_{\omega}(t, \tau)}
$$

\section{Estimates on $C_{j}, D_{j}$}

Clearly,

$$
C_{j} \lesssim \frac{1}{j^{1 / 2}}\left\|\partial_{x}^{j} \omega\right\|_{L^{2}\left(\mathbb{T} \times K^{a}\right)}, \quad\left\|C_{j}\right\|_{l^{2}(\tau)} \lesssim \sqrt{E_{\omega}(t, \tau)}
$$

and after integration by parts

$$
\begin{aligned}
& D_{j} \lesssim \frac{1}{j^{1 / 2}}\left\|\partial_{x}^{j} \omega\right\|_{L^{2}\left(\mathbb{T} \times K^{a}\right)}\left(\left\|h_{j}\right\|_{L^{2}\left(\mathbb{T} \times \mathbb{R}_{+}\right)}+\left\|\partial_{y} h_{j}\right\|_{L^{2}\left(\mathbb{T} \times \mathbb{R}_{+}\right)}\right) \\
& \left\|D_{j}\right\|_{l^{1}(\tau)} \lesssim \sqrt{E_{\omega}(t, \tau)}\left(\sqrt{E_{h}(t, \tau)}+\sqrt{D_{h}(t, \tau)}\right) \\
& \lesssim \eta D_{h}(t, \tau)+C_{\eta} E_{\omega}(t, \tau) .
\end{aligned}
$$

Let us stress that Lemma 2 allowed us to control $E_{h}$ by $E_{\omega}$.

\section{Estimates on $E_{j}$}

It remains to handle the bad term:

$$
\begin{aligned}
E_{j} & =\int_{\mathbb{T} \times \mathbb{R}_{+}}\left(\chi^{a}\right)^{2} \partial_{x}^{j} v \partial_{x}^{j} \omega=-\int_{\mathbb{T} \times \mathbb{R}_{+}} \partial_{y}\left(\chi^{a}\right)^{2} \partial_{x}^{j} v \partial_{x}^{j} u-\int_{\mathbb{T} \times \mathbb{R}_{+}}\left(\chi^{a}\right)^{2} \partial_{x}^{j} \partial_{y} v \partial_{x}^{j} u \\
& =\int_{\mathbb{T} \times \mathbb{R}_{+}} \partial_{y}\left(\chi^{a}\right)^{2} \partial_{x}\left(\int_{0}^{y} \partial_{x}^{j} u\right) \partial_{x}^{j} u+\int_{\mathbb{T} \times \mathbb{R}_{+}}\left(\chi^{a}\right)^{2} \partial_{x} \frac{\left(\partial_{x}^{j} u\right)^{2}}{2} \\
& =-\int_{\mathbb{T} \times \mathbb{R}_{+}} \partial_{y}\left(\chi^{a}\right)^{2} \int_{0}^{y} \partial_{x}^{j} u \partial_{x}^{j+1} u-\int_{\mathbb{T} \times \mathbb{R}_{+}}\left(\partial_{x y}\left(\chi^{a}\right)^{2} \int_{0}^{y} \partial_{x}^{j} u \partial_{x}^{j} u+\partial_{x}\left(\chi^{a}\right)^{2} \frac{\left(\partial_{x}^{j} u\right)^{2}}{2}\right) \\
& :=E_{j}^{1}+E_{j}^{2}
\end{aligned}
$$

Clearly,

$$
\left\|E_{j}^{2}\right\|_{l^{1}(\tau)} \lesssim\|\| \partial_{x}^{j} u\left\|_{L^{2}\left(\mathbb{T} \times K^{a}\right)}\right\|_{l^{2}(\tau)}^{2} \lesssim\|\| \partial_{x}^{j} \omega\left\|_{L^{2}\left(\mathbb{T} \times K^{a}\right)}\right\|_{l^{2}(\tau)}^{2} \lesssim E_{\omega}(t, \tau)
$$

with Poincaré inequality allowing to go from $u$ to $\omega$. 
We are left with the treatment of $E_{j}^{1}$. A crucial remark is that for $\varepsilon>0$ small enough, for all $t \in[0, T]$, the integrand in $E_{j}^{1}=E_{j}^{1}(t)$ is supported in

$$
\{|y-a| \geq \varepsilon\}:=\{(x, y),|y-a(t, x)| \geq \varepsilon\} .
$$

Indeed, $\chi^{a}$ is one in a neighborhood of $y=a$ (moreover, $\chi^{a}=0$ for $y \geq 3$ ). We then recall the decomposition stated in Lemma 3 , for all $j$,

$$
\partial_{x}^{j} u=u_{g}^{j}+C_{j} 1_{\{y>a\}} \omega
$$

with

$$
\begin{aligned}
& u_{g}^{j}:=\omega(t, x, y) \int_{0}^{y}\left(\psi+\frac{(1-\psi)}{\omega}\right)^{-1} \frac{g_{j}}{\omega^{2}} \text { in }\{y<a\}, \\
& u_{g}^{j}:=\omega(t, x, y) \int_{3}^{y}\left(\psi+\frac{(1-\psi)}{\omega}\right)^{-1} \frac{g_{j}}{\omega^{2}} \text { in }\{y>a\} .
\end{aligned}
$$

Note that

$$
\left\|u_{g}^{j}\right\|_{L^{2}(\{\varepsilon \leq|y-a|, y \leq 3\})} \lesssim\left\|g_{j}\right\|_{L^{2}(\{y \leq 3\})} .
$$

For $y<a$, we write

$$
\begin{aligned}
\left|\int_{\{y<a\}} \partial_{y}\left(\chi^{a}\right)^{2}\left(\int_{0}^{y} \partial_{x}^{j} u\right) \partial_{x}^{j+1} u\right| & =\left|\int_{\{y<a\}} \partial_{y}\left(\chi^{a}\right)^{2}\left(\int_{0}^{y} u_{g}^{j}\right) u_{g}^{j+1}\right| \\
& \lesssim\left\|g_{j}\right\|_{L^{2}(\{y \leq 3\})}\left\|g_{j+1}\right\|_{L^{2}(\{y \leq 3\})} \\
& \lesssim\left\|g_{j}\right\|_{L^{2}(\{y \leq 3\})}\left\|\partial_{x}^{j+1} \omega\right\|_{L^{2}(\{y \leq 3\})} .
\end{aligned}
$$

For $y>a$, we rather write

$$
\begin{aligned}
& \left|\int_{\{y>a\}} \partial_{y}\left(\chi^{a}\right)^{2}\left(\int_{0}^{y} \partial_{x}^{j} u\right) \partial_{x}^{j+1} u\right|=\left|\int_{\{y>a\}} \partial_{y}\left(\chi^{a}\right)^{2}\left(\int_{0}^{y} u_{g}^{j}\right) \partial_{x}^{j+1} u+\int_{\{y>a\}} C_{j}\left(\int_{a}^{y} \omega\right) \partial_{x}^{j+1} u\right| \\
& \lesssim\left\|\int_{0}^{y} u_{g}^{j}\right\|_{L^{2}(\{a+\varepsilon \leq y \leq 3\})}\left\|\partial_{x}^{j+1} \omega\right\|_{L^{2}\left(\mathbb{T} \times \mathbb{R}_{+}\right)}+\left|\int_{\{y>a\}} C_{j}\left(\int_{a}^{y} \omega\right) \partial_{x}^{j+1} u\right| .
\end{aligned}
$$

Note that although $y$ is away from $a$, the function $\int_{0}^{y} u_{g}^{j}$ involves values of $u_{g}^{j}$ near the critical curve $y=a$. As the quantity $\frac{g_{j}}{\omega^{2}}$ (involved in the definition of $u_{g}^{j}$ ) degenerates at $y=a$, a control like (3.38) is not obvious. Still, we claim:

\section{Lemma 6}

$$
\left\|\int_{0}^{y} u_{g}^{j}\right\|_{L^{2}(\{a+\varepsilon \leq y \leq 3\})} \lesssim\left\|g_{j}\right\|_{L^{2}(\{y \leq 3\})} \lesssim\left\|\partial_{x}^{j} \omega\right\|_{L^{2}\left(\mathbb{T} \times \mathbb{R}_{+}\right)} .
$$

Furthermore, we have

$$
\left\|C_{j}\right\|_{L^{2}(\mathbb{T})} \lesssim\left\|\partial_{x}^{j} \omega\right\|_{L^{2}\left(\mathbb{T} \times \mathbb{R}_{+}\right)}, \quad\left\|\partial_{x} C_{j}\right\|_{L^{2}(\mathbb{T})} \lesssim\left\|\partial_{x}^{j+1} \omega\right\|_{L^{2}\left(\mathbb{T} \times \mathbb{R}_{+}\right)}+\left\|\partial_{x}^{j} \omega\right\|_{L^{2}\left(\mathbb{T} \times \mathbb{R}_{+}\right)} .
$$


Finally, using above bounds and the decomposition

$$
\partial_{x}^{j+1} u=\partial_{x} u_{g}^{j}+\partial_{x} C_{j} \omega+C_{j} \partial_{x} \omega
$$

we end up with

$$
\begin{aligned}
& \left|\int_{\{y>a\}} \partial_{y}\left(\chi^{a}\right)^{2}\left(\int_{0}^{y} \partial_{x}^{j} u\right) \partial_{x}^{j+1} u\right| \\
& \lesssim\left\|g_{j}\right\|_{L^{2}(\{y \leq 3\})}\left\|\partial_{x}^{j+1} \omega\right\|_{L^{2}\left(\mathbb{T} \times \mathbb{R}_{+}\right)}+\left|\int_{\mathbb{T} \times \mathbb{R}_{+}} \partial_{y}\left(\chi^{a}\right)^{2} C_{j}\left(\int_{a}^{y} \omega\right) \partial_{x} u_{g}^{j}\right| \\
& +\left|\int_{\mathbb{T} \times \mathbb{R}_{+}} \partial_{y}\left(\chi^{a}\right)^{2} \partial_{x} \frac{C_{j}^{2}}{2} \omega\left(\int_{0}^{y} \omega\right)\right|+\left\|\partial_{x}^{j} \omega\right\|_{L^{2}\left(\mathbb{T} \times \mathbb{R}_{+}\right)}^{2} \\
& \lesssim\left\|g_{j}\right\|_{L^{2}(\{y \leq 3\})}\left\|\partial_{x}^{j+1} \omega\right\|_{L^{2}\left(\mathbb{T} \times \mathbb{R}_{+}\right)}+\left\|\partial_{x}^{j} \omega\right\|_{L^{2}\left(\mathbb{T} \times \mathbb{R}_{+}\right)}^{2}
\end{aligned}
$$

after integration by parts of the integral terms at the right-hand side. The structure of the second integral term is crucial. Indeed, the term involving the product $\left(\partial_{x} C_{j} \omega\right)\left(C_{j} \int_{a}^{y} \omega\right)$ can be integrated by parts. Note that a more general bilinear term in $C_{j}$ and $\partial_{x} C_{j}$ would generically involve an upper bound like $\left\|C_{j}\right\|_{L^{2}}\left\|\partial_{x} C_{j}\right\|_{L^{2}}$. Such bound would ruin our strategy, based on anisotropic energy.

From the previous bound, from inequality (3.39) and Cauchy-Schwarz in $l^{2}(\tau)$, we get

$$
\left\|E_{j}^{1}\right\|_{l^{1}(\tau)} \lesssim\left\|\frac{1}{j^{5 / 4}}\right\| \partial_{x}^{j+1} \omega\left\|_{L^{2}\left(\mathbb{T} \times \mathbb{R}_{+}\right)}\right\|_{l^{2}(\tau)}\left\|j^{5 / 4}\right\| g_{j}\left\|_{L^{2}(\{y \leq 3\})}\right\|_{l^{2}(\tau)}+\|\| \partial_{x}^{j} \omega\left\|_{L^{2}\left(\mathbb{T} \times \mathbb{R}_{+}\right)}\right\|_{l^{2}(\tau)}^{2} .
$$

Here, we remark that

$$
\begin{aligned}
\left\|\frac{1}{j^{5 / 4}}\right\| \partial_{x}^{j+1} \omega\left\|_{L^{2}\left(\mathbb{T} \times \mathbb{R}_{+}\right)}\right\|_{l^{2}(\tau)}^{2} & =\sum_{j=0}^{+\infty} \frac{1}{j^{5 / 2}}\left(\tau^{j}(j !)^{-7 / 4}(j+1)^{10}\right)^{2}\|\omega\|_{\mathcal{H}_{\gamma}^{j+1}}^{2} \\
& \lesssim \sum_{j=1}^{+\infty} j\left(\tau^{j}(j !)^{-7 / 4}(j+1)^{10}\right)^{2}\|\omega\|_{\mathcal{H}_{\gamma}^{j}}^{2} \\
& \lesssim\|\sqrt{j}\| \omega\left\|_{\mathcal{H}_{\gamma}^{j}}\right\|_{l^{2}(\tau)}^{2} \lesssim \partial_{\tau} E_{\omega}(t, \tau) .
\end{aligned}
$$

To conclude, it remains to evaluate the $l^{2}(\tau)$ norm of $j^{5 / 4}\left\|g_{j}\right\|_{L^{2}(\{y \leq 3\})}$. We use the following bound, to be proved in appendix

Lemma 7 For all $\alpha \geq 0$,

$$
\|\| j^{\alpha / 4} g_{j}\left\|_{L^{2}(\{y \leq 3\})}\right\|_{l^{2}(\tau)} \lesssim\|\| j^{\alpha / 4} \tilde{g}_{j}\left\|_{L^{2}(\{y \leq 3\})}\right\|_{l^{2}(\tau)}+\left\|j^{(\alpha-3) / 4}\right\| \omega\left\|_{\mathcal{H}_{\gamma}^{j}}\right\|_{l^{2}(\tau)} .
$$

For $\alpha=5$, we find

$$
\|\| j^{5 / 4} g_{j}\left\|_{L^{2}(\{y \leq 3\})}\right\|_{l^{2}(\tau)} \lesssim \sqrt{\partial_{\tau} E_{g}^{2}(t, \tau)}+\sqrt{\partial_{\tau} E_{\omega}(t, \tau)} .
$$

We inject this bound and bound (3.43) in the estimate of $\left\|E_{j}^{1}\right\|_{l^{1}(\tau)}$, we get

$$
\left\|E_{j}^{1}\right\|_{l^{1}(\tau)} \lesssim \sqrt{\partial_{\tau} E_{\omega}(t, \tau)} \sqrt{\partial_{\tau} E_{g}^{2}(t, \tau)}+\partial_{\tau} E_{\omega}(t, \tau) \lesssim \partial_{\tau} E_{\omega}(t, \tau)+\partial_{\tau} E_{g}^{2}(t, \tau)
$$


Combination of this inequality and inequality (3.36) yields

$$
\left\|E_{j}\right\|_{l^{1}(\tau)} \lesssim \partial_{\tau} E_{\omega}(t, \tau)+\partial_{\tau} E_{g}^{2}(t, \tau)
$$

Collecting (3.32)-(3.33)-(3.34)-(3.35)-(3.47) leads to

$$
\partial_{t} E_{h}(t, \tau)+D_{h}(t, \tau) \lesssim \eta D_{h}(t, \tau)+C_{\eta} \partial_{\tau} E_{\omega}(t, \tau)+\partial_{\tau} E_{g}^{2}(t, \tau)
$$

Taking $\eta$ small enough yields Proposition 2 .

\subsection{Estimate of the (second) monotonicity energy}

The control of $E_{h}$, performed in the previous subsection, has relied on the so-called monotonicity energy $E_{g}^{2}$. Its time variations $\partial_{t} E_{g}^{2}$ need in turn to be controlled by $\partial_{\tau} \mathcal{E}$, in order to obtain a closed estimate. The main difficulty comes from the extra factor $j^{3 / 2}$ in the definition of $E_{g}^{2}$ : indeed, naive energy bounds would involve the $l^{2}(\tau)$ norm of $\left(j^{5 / 2}\|\omega\|_{\mathcal{H}_{\gamma}^{j}}\right)_{j \in \mathbb{N}}$, which is not controlled by $\sqrt{\partial_{\tau} \mathcal{E}}$.

To get a good estimate, we must take advantage of cancellation properties in the equation for

$$
\tilde{g}_{j}(t, x, y)=\partial_{x}^{j-5}\left(\omega \partial_{x}^{5} \omega-\partial_{y} \omega \partial_{x}^{5} u\right) .
$$

As mentioned in section 2, this kind of cancellations was used for the first time in paper [17], in the case of monotonic data: this paper shows indeed some Sobolev stability, with special role played by

$$
g_{j}:=\omega \partial_{x}^{j} \omega-\partial_{y} \omega \partial_{x}^{j} u
$$

However, in our setting, there is a technical difficulty with using $g_{j}$ (or the $g_{j}$ defined in (2.12), better suited to large $y$ ). Broadly speaking, the equation for $g_{j}$ involves the commutator term $j \partial_{x}^{j-1} v \partial_{x} \partial_{y} \omega$, with a factor $j$ in front. This factor is harmless at the Sobolev level (finite $j$ ), but is annoying at the Gevrey level, for which behaviour at large $j$ is important. This is why we rather use $\tilde{g}_{j}$ than $g_{j}: \mathcal{C}_{j}$ is somehow replaced by $5 \partial_{x}^{j-1} v \partial_{x} \partial_{y} \omega$, with no bad factor. Roughly, instead of differentiating $j$ times the velocity and vorticity equations, and combining them to obtain cancellations, we go the reverse way: we first combine the equations and then differentiate, so as to benefit from "earlier" cancellations.

We shall prove

Proposition 3 Under the assumptions of Theorem 2, we have

$$
\partial_{t} E_{g}^{2}(t, \tau)+D_{g}^{2}(t, \tau) \leq C\left(\partial_{\tau} E_{\omega}(t, \tau)+\partial_{\tau} E_{g}^{2}(t, \tau)+D_{h}(t, \tau)\right), \quad C>0 .
$$

We recall that $D_{h}$ was defined in (3.29), whereas

$$
D_{g}^{2}(t, \tau):=\sum_{j \in \mathbb{N}}\left(\tau^{j}(j !)^{-7 / 4}(j+1)^{10}\right)^{2} j^{3 / 2}\left\|\partial_{y} \tilde{g}_{j}(t, \cdot)\right\|_{L^{2}\left(\mathbb{T} \times \mathbb{R}_{+}\right)}^{2}
$$

As usual, the starting point is to derive an equation for $\tilde{g}_{j}, j \geq 5$. First, we apply $\omega \partial_{5}^{x}$ to the vorticity equation, $\partial_{y} \omega \partial_{x}^{5}$ to the velocity equation, and subtract one from the other: we 
obtain

$$
\begin{aligned}
\partial_{t} \tilde{g}_{5}+u \partial_{x} \tilde{g}_{5} & +v \partial_{y} \tilde{g}_{5}-\partial_{y}^{2} \tilde{g}_{5}=-\sum_{k=1}^{5}\left(\begin{array}{l}
5 \\
k
\end{array}\right) \partial_{x}^{k} u \bar{g}_{5-k+1}-\sum_{k=1}^{4}\left(\begin{array}{l}
5 \\
k
\end{array}\right) \partial_{x}^{k} v \hat{g}_{5-k+1} \\
& -\left(\partial_{t}+u \partial_{x}+v \partial_{y}-\partial_{y}^{2}\right) \partial_{y} \omega \partial_{x}^{5} u+2 \partial_{y}^{2} \omega \partial_{x}^{5} \omega-2 \partial_{y} \omega \partial_{y} \partial_{x}^{5} \omega:=\sum_{i=1}^{5} \mathcal{C}_{i}
\end{aligned}
$$

with

$$
\begin{aligned}
& \bar{g}_{k}:=\omega \partial_{x}^{k} \omega-\partial_{y} \omega \partial_{x}^{k} u, \\
& \hat{g}_{k}:=\omega \partial_{x}^{k-1} \partial_{y} \omega-\partial_{y} \omega \partial_{x}^{k-1} \omega, \quad 1 \leq k
\end{aligned}
$$

We finally apply $\partial_{x}^{j-5}$ to the equation, which yields

$$
\partial_{t} \tilde{g}_{j}+u \partial_{x} \tilde{g}_{j}+v \partial_{y} \tilde{g}_{j}-\partial_{y}^{2} \tilde{g}_{j}=\left[u \partial_{x}, \partial_{x}^{j-5}\right] \tilde{g}_{5}+\left[v \partial_{y}, \partial_{x}^{j-5}\right] \tilde{g}_{5}+\sum_{i=1}^{5} \partial_{x}^{j-5} \mathcal{C}_{i}
$$

We perform an energy estimate, multiply by $j^{3 / 2}\left(\tau^{j}(j !)^{-7 / 4}(j+1)^{10}\right)^{2}$ and sum over $j$ :

$\partial_{t} E_{g}^{2}(t, \tau)+D_{g}^{2}(t, \tau) \leq\left(\left\|A_{j}\right\|_{l^{2}(\tau)}+\left\|B_{j}\right\|_{l^{2}(\tau)}+\sum_{i=1}^{4}\left\|C_{i, j}\right\|_{l^{2}(\tau)}\right) \sqrt{\partial_{\tau} E_{g}^{2}(t, \tau)}+\left\|\max \left(D_{j}, 0\right)\right\|_{l^{1}(\tau)}$

with

$$
\begin{aligned}
A_{j} & :=j^{1 / 4}\left\|\left[u \partial_{x}, \partial_{x}^{j-5}\right] \tilde{g}_{5}\right\|_{L^{2}\left(\mathbb{T} \times \mathbb{R}_{+}\right)}, \quad B_{j}:=j^{1 / 4}\left\|\left[v \partial_{y}, \partial_{x}^{j-5}\right] \tilde{g}_{5}\right\|_{L^{2}\left(\mathbb{T} \times \mathbb{R}_{+}\right)}, \\
C_{i, j} & :=j^{1 / 4}\left\|\partial_{x}^{j-5} \mathcal{C}_{i}\right\|_{L^{2}\left(\mathbb{T} \times \mathbb{R}_{+}\right)}, \quad D_{j}:=j^{3 / 2} \int_{\mathbb{T} \times \mathbb{R}_{+}} \partial_{x}^{j-5} \mathcal{C}_{5} \tilde{g}_{j} .
\end{aligned}
$$

Note that

$$
\left.\partial_{y} \tilde{g}_{j}\right|_{y=0}=\left.\partial_{x}^{j-5}\left(\partial_{y} \omega \partial_{x}^{5} \omega-\partial_{y}^{2} \omega \partial_{x}^{5} u\right)\right|_{y=0}=0 .
$$

Indeed, $\left.\partial_{x}^{5} u\right|_{y=0}=0$, and $\left.\partial_{y} \omega\right|_{y=0}=\left.\partial_{y}^{2} u\right|_{y=0}=0$, evaluating the Prandtl equation at $y=0$. In particular, there is no boundary term due to the integration by parts.

\section{Estimate on $A_{j}$}

We find

$$
\left[u \partial_{x}, \partial_{x}^{j-5}\right] \tilde{g}_{5}=\sum_{k=1}^{j-5}\left(\begin{array}{c}
j-5 \\
k
\end{array}\right) \partial_{x}^{k} u \tilde{g}_{j-k+1}
$$

Thus,

$$
A_{j} \leq j^{1 / 4} \sum_{k=1}^{j / 2}\left(\begin{array}{c}
j-5 \\
k
\end{array}\right)\left\|\partial_{x}^{k} u\right\|_{L^{\infty}}\left\|\tilde{g}_{j-k+1}\right\|_{L^{2}}+j^{1 / 4} \sum_{k=j / 2}^{j-5}\left\|\partial_{x}^{k} u\right\|_{L^{2}}\left\|\tilde{g}_{j-k+1}\right\|_{L^{\infty}}:=A_{j}^{1}+A_{j}^{2} .
$$

Using like before (A.3) and (A.1), we get

$$
\left\|\partial_{x}^{k} u\right\|_{L^{\infty}} \lesssim \sum_{m=0}^{1}\|\omega\|_{\mathcal{H}_{\gamma}^{k+m}}, \quad\left\|\tilde{g}_{j-k+1}\right\|_{L^{\infty}} \lesssim \sum_{m=1}^{2}\left\|\tilde{g}_{j-k+m}\right\|_{L^{2}}+\sum_{m=2}^{3}\left\|\partial_{y} \tilde{g}_{j-k-1+m}\right\|_{L^{2}} .
$$


Hence (set $k^{\prime}:=k-1$, drop the prime)

$$
A_{j}^{1} \lesssim j^{1 / 4} \sum_{k=0}^{j / 2-1}\left(\begin{array}{l}
j-5 \\
k+1
\end{array}\right) \sum_{m=1}^{2}\|\omega\|_{\mathcal{H}_{\gamma}^{k+m}}\left\|\tilde{g}_{j-k}\right\|_{L^{2}} \lesssim \sum_{k=0}^{j / 2}\left(\begin{array}{l}
j \\
k
\end{array}\right) \sum_{m=1}^{2}\|\omega\|_{\mathcal{H}_{\gamma}^{k+m}}(j-k)^{1 / 4}\left\|\tilde{g}_{j-k}\right\|_{L^{2}}
$$

using that for $0 \leq k \leq j / 2$, one has $\left(\begin{array}{l}j-5 \\ k+1\end{array}\right) \lesssim\left(\begin{array}{l}j \\ k\end{array}\right)$ and $j^{1 / 4} \lesssim(j-k)^{1 / 4}$. Hence, by Lemma 1

$$
\begin{aligned}
\left\|A_{j}^{1}\right\|_{l^{2}(\tau)} & \lesssim\|\| \omega\left\|_{\mathcal{H}_{\gamma}^{j}}\right\|_{l^{2}(\tau)}\left\|j^{1 / 4}\right\| \tilde{g}_{j}\left\|_{L^{2}\left(\mathbb{T} \times \mathbb{R}_{+}\right)}\right\|_{l^{2}(\tau)} \\
& \lesssim \sqrt{E_{\omega}(t, \tau)} \sqrt{\partial_{\tau} E_{g}^{2}(t, \tau)} \lesssim \sqrt{\partial_{\tau} E_{g}^{2}(t, \tau)}
\end{aligned}
$$

Finally,

$$
\begin{aligned}
A_{j}^{2} & \lesssim j^{1 / 4} \sum_{k=j / 2}^{j-5}\left(\begin{array}{c}
j-5 \\
k
\end{array}\right)\left\|\partial_{x}^{k} u\right\|_{L^{2}} \sum_{m=1}^{2}\left\|\tilde{g}_{j-k+m}\right\|_{L^{2}}+\sum_{m=2}^{3}\left\|\partial_{y} \tilde{g}_{j-k-1+m}\right\|_{L^{2}} \\
& \lesssim \sum_{k=j / 2}^{j}\left(\begin{array}{l}
j \\
k
\end{array}\right) k^{1 / 4}\|\omega\|_{\mathcal{H}_{\gamma}^{k}}\left(\sum_{m=1}^{2}\left\|\tilde{g}_{j-k+m}\right\|_{L^{2}}+\sum_{m=2}^{3}\left\|\partial_{y} \tilde{g}_{j-k-1+m}\right\|_{L^{2}}\right)
\end{aligned}
$$

using that for $j / 2 \leq k \leq j-5$, one has $\left(\begin{array}{c}j-5 \\ k\end{array}\right) \lesssim\left(\begin{array}{c}j \\ k\end{array}\right)$ and $j^{1 / 4} \lesssim k^{1 / 4}$. Hence,

$$
\left\|A_{j}^{2}\right\|_{l^{2}(\tau)} \leq\left(\|\| \tilde{g}_{j}\left\|_{L^{2}\left(\mathbb{T} \times \mathbb{R}_{+}\right)}\right\|_{l^{2}(\tau)}+\|\| \partial_{y} \tilde{g}_{j-1}\left\|_{L^{2}\left(\mathbb{T} \times \mathbb{R}_{+}\right)}\right\|_{l^{2}(\tau)}\right)\left\|j^{1 / 4}\right\| \omega\left\|_{\mathcal{H}_{\gamma}^{j}}\right\|_{l^{2}(\tau)} .
$$

We conclude by using

Lemma 8 For all $\alpha \geq 0$, for all $l=0,1,2$,

$$
\left\|j^{\alpha}\right\| \partial_{y}^{l} \tilde{g}_{j-l}\left\|_{L^{2}\left(\mathbb{T} \times \mathbb{R}_{+}\right)}\right\|_{l^{2}(\tau)} \lesssim\left\|j^{\alpha}\right\| \omega\left\|_{\mathcal{H}_{\gamma}^{j}}\right\|_{l^{2}(\tau)}, \quad \alpha \geq 0 .
$$

For a proof, see the appendix. We get

$$
\left\|A_{j}^{2}\right\|_{l^{2}(\tau)} \lesssim \sqrt{E_{\omega}(t, \tau)} \sqrt{\partial_{\tau} E_{\omega}(t, \tau)} \lesssim \sqrt{\partial_{\tau} E_{\omega}(t, \tau)}
$$

and combining with (3.54),

$$
\left\|A_{j}\right\|_{l^{2}(\tau)} \lesssim \sqrt{\partial_{\tau} E_{g}^{2}(t, \tau)}+\sqrt{\partial_{\tau} E_{\omega}(t, \tau)} .
$$

\section{Estimate on $B_{j}$}

Leibniz formula gives

$$
\left[v \partial_{y}, \partial_{x}^{j-5}\right] \tilde{g}_{5}=\sum_{k=1}^{j-5}\left(\begin{array}{c}
j-5 \\
k
\end{array}\right) \partial_{x}^{k} v \partial_{y} \tilde{g}_{j-k} .
$$

Proceeding as before, we get easily

$$
\begin{aligned}
B_{j} & \leq j^{1 / 4} \sum_{k=1}^{j / 2}\left(\begin{array}{c}
j-5 \\
k
\end{array}\right) \sum_{m=1}^{2}\|\omega\|_{\mathcal{H}_{\gamma}^{k+m}}\left\|\partial_{y} \tilde{g}_{j-k}\right\|_{L^{2}} \\
& +j^{1 / 4} \sum_{k=j / 2}^{j-5}\|\omega\|_{\mathcal{H}_{\gamma}^{k+1}}\left(\sum_{m=1}^{2}\left\|\partial_{y} \tilde{g}_{j-k-1+m}\right\|_{L^{2}}+\sum_{m=2}^{3}\left\|\partial_{y}^{2} \tilde{g}_{j-k-2+m}\right\|_{L^{2}}\right):=B_{j}^{1}+B_{j}^{2}
\end{aligned}
$$


Then, using that $\left(\begin{array}{c}j-5 \\ k\end{array}\right) \lesssim\left(\begin{array}{c}j \\ k\end{array}\right), j^{1 / 4} \lesssim(j-k)^{1 / 4}$ for $0 \leq k \leq j / 2$, we find

$$
B_{j}^{1} \leq \sum_{k=0}^{j / 2}\left(\begin{array}{l}
j \\
k
\end{array}\right) \sum_{m=1}^{2}\|\omega\|_{\mathcal{H}_{\gamma}^{k+m}}(j-k)^{1 / 4}\left\|\partial_{y} \tilde{g}_{j-k}\right\|_{L^{2}}
$$

so that

$$
\left\|B_{j}^{1}\right\|_{l^{2}(\tau)} \lesssim \sqrt{E_{\omega}(t, \tau)} \sqrt{\left\|j^{1 / 4}\right\| \partial_{y} \tilde{g}_{j}\left\|_{L^{2}\left(\mathbb{T} \times \mathbb{R}_{+}\right)}\right\|_{l^{2}(\tau)}} \lesssim \sqrt{D_{g}^{2}(t, \tau)}
$$

Also,

$$
\begin{aligned}
B_{j}^{2} & \lesssim j^{1 / 4} \sum_{k=j / 2+1}^{j-4}\left(\begin{array}{c}
j-5 \\
k-1
\end{array}\right)\|\omega\|_{\mathcal{H}_{\gamma}^{k}}\left(\sum_{m=2}^{3}\left\|\partial_{y} \tilde{g}_{j-k-1+m}\right\|_{L^{2}}+\sum_{m=3}^{4}\left\|\partial_{y}^{2} \tilde{g}_{j-k-2+m}\right\|_{L^{2}}\right) \\
& \lesssim \sum_{k=j / 2}^{j}\left(\begin{array}{l}
j \\
k
\end{array}\right)\left(k^{1 / 4}\|\omega\|_{\mathcal{H}_{\gamma}^{k}}\right)\left(\sum_{m=2}^{3}\left\|\partial_{y} \tilde{g}_{j-k-1+m}\right\|_{L^{2}}+\sum_{m=3}^{4}\left\|\partial_{y}^{2} \tilde{g}_{j-k-2+m}\right\|_{L^{2}}\right)
\end{aligned}
$$

using that $\left(\begin{array}{l}j-5 \\ k-1\end{array}\right) \lesssim\left(\begin{array}{l}j \\ k\end{array}\right), j^{1 / 4} \lesssim k^{1 / 4}$ in the range $j / 2 \leq k \leq j-4$. Hence,

$$
\begin{aligned}
\left\|B_{j}^{2}\right\|_{l^{2}(\tau)} & \lesssim \sqrt{\partial_{\tau} E_{\omega}(t, \tau)}\left(\|\| \partial_{y} \tilde{g}_{j-1}\left\|_{L^{2}\left(\mathbb{T} \times \mathbb{R}_{+}\right)}\right\|_{l^{2}(\tau)}+\|\| \partial_{y}^{2} \tilde{g}_{j-2}\left\|_{L^{2}\left(\mathbb{T} \times \mathbb{R}_{+}\right)}\right\|_{l^{2}(\tau)}\right) \\
& \lesssim \sqrt{\partial_{\tau} E_{\omega}(t, \tau)} \sqrt{E_{\omega}(t, \tau)} \lesssim \sqrt{\partial_{\tau} E_{\omega}(t, \tau)}
\end{aligned}
$$

where we have applied Lemma 8 to go from the first to the second line. Gathering the estimates on $B_{j}^{1}$ and $B_{j}^{2}$,

$$
\left\|B_{j}\right\|_{l^{2}(\tau)} \lesssim \sqrt{D_{g}^{2}(t, \tau)}+\sqrt{\partial_{\tau} E_{\omega}(t, \tau)}
$$

Estimates on $C_{i, j}, 1 \leq i \leq 2$

The quantities $C_{1, j}$ and $C_{2, j}$ involve commutators with the nonlinearities. As we have manipulated several times such commutators, we shall not detail the computation of their $l^{2}(\tau)$ norms. One can check that

$$
\begin{aligned}
\left\|C_{1, j}\right\|_{l^{2}(\tau)} & \lesssim\|\| \omega\left\|_{\mathcal{H}_{\gamma}^{j}}\right\|_{l^{2}(\tau)} \sum_{m=1}^{5}\left\|j^{1 / 4}\right\| \partial_{x}^{j-5} \bar{g}_{m}\left\|_{L^{2}\left(\mathbb{T} \times \mathbb{R}_{+}\right)}\right\|_{l^{2}(\tau)} \\
& +\left\|j^{1 / 4}\right\| \omega\left\|_{\mathcal{H}_{\gamma}^{j}}\right\|_{l^{2}(\tau)} \sum_{m=1}^{5}\left(\|\| \partial_{x}^{j-5} \bar{g}_{m}\left\|_{L^{2}\left(\mathbb{T} \times \mathbb{R}_{+}\right)}\right\|_{l^{2}(\tau)}+\|\| \partial_{y} \partial_{x}^{j-6} \bar{g}_{m}\left\|_{L^{2}\left(\mathbb{T} \times \mathbb{R}_{+}\right)}\right\|_{l^{2}(\tau)}\right) \\
& \lesssim \sqrt{\partial_{\tau} E_{\omega}(t, \tau)}
\end{aligned}
$$

Here, we use implicitly the bound

$$
\left\|j^{\alpha}\right\| \partial_{y}^{l} \partial_{x}^{j-5-l} \bar{g}_{m}\left\|_{L^{2}\left(\mathbb{T} \times \mathbb{R}_{+}\right)}\right\|_{l^{2}(\tau)} \lesssim\left\|j^{\alpha}\right\| \omega\left\|_{\mathcal{H}_{\gamma}^{j}}\right\|_{l^{2}(\tau)}
$$

which is valid for all $1 \leq m \leq 5, \alpha \geq 0$ and $0 \leq l \leq 2$. Note that the case $m=5$ corresponds to Lemma 8 . This inequality holds a fortiori for $m \leq 4$, as less $x$-derivatives are involved. Actually, one can even show that

$$
\text { for } 1 \leq m \leq 4, \quad\left\|j^{\alpha}\right\| \partial_{y}^{l} \partial_{x}^{j-4-l} \bar{g}_{m}\left\|_{L^{2}\left(\mathbb{T} \times \mathbb{R}_{+}\right)}\right\|_{l^{2}(\tau)} \lesssim\left\|j^{\alpha}\right\| \omega\left\|_{\mathcal{H}_{\gamma}^{j}}\right\|_{l^{2}(\tau)}
$$


We leave the details to the reader.

As regards $C_{2, j}$,

$$
\begin{aligned}
\left\|C_{2, j}\right\|_{l^{2}(\tau)} & \lesssim\|\| \omega\left\|_{\mathcal{H}_{\gamma}^{j}}\right\|_{l^{2}(\tau)} \sum_{m=1}^{4}\left\|j^{1 / 4}\right\| \partial_{x}^{j-5} \hat{g}_{m}\left\|_{L^{2}\left(\mathbb{T} \times \mathbb{R}_{+}\right)}\right\|_{l^{2}(\tau)} \\
& +\left\|j^{1 / 4}\right\| \omega\left\|_{\mathcal{H}_{\gamma}^{j}}\right\|_{l^{2}(\tau)} \sum_{m=2}^{5}\left(\|\| \partial_{x}^{j-5} \hat{g}_{m}\left\|_{L^{2}\left(\mathbb{T} \times \mathbb{R}_{+}\right)}\right\|_{l^{2}(\tau)}+\|\| \partial_{y} \partial_{x}^{j-6} \hat{g}_{m}\left\|_{L^{2}\left(\mathbb{T} \times \mathbb{R}_{+}\right)}\right\|_{l^{2}(\tau)}\right) \\
& \lesssim \sqrt{\partial_{\tau} E_{\omega}(t, \tau)}
\end{aligned}
$$

Again, we have applied inequalities of type (3.61)-(3.62), with $\bar{g}$ replaced by $\hat{g}$.

\section{Estimate on $C_{4, j}$}

Once more, one can follow closely the previous ideas, and derive

$$
\left\|C_{4, j}\right\|_{l^{2}(\tau)} \lesssim \sqrt{E_{\omega}(t, \tau)}\left\|j^{1 / 4}\right\| \omega\left\|_{\mathcal{H}_{\gamma}^{j}}\right\|_{l^{2}(\tau)} \lesssim \sqrt{\partial_{\tau} E_{\omega}(t, \tau)}
$$

\section{Estimate on $C_{3, j}$}

We remind that

$$
\left(\partial_{t}+u \partial_{x}+v \partial_{y}-\partial_{y}^{2}\right) \omega=0
$$

so that

$$
\left(\partial_{t}+u \partial_{x}+v \partial_{y}-\partial_{y}^{2}\right) \partial_{y} \omega=-\partial_{y} u \partial_{x} \omega-\partial_{y} v \partial_{y} \omega=-\partial_{y} u \partial_{x} \omega+\partial_{x} u \partial_{y} \omega
$$

Hence,

$$
\partial_{x}^{j-5} \mathcal{C}_{3, j}=\sum_{k=0}^{j-5}\left(\begin{array}{c}
j-5 \\
k
\end{array}\right) \partial_{x}^{k}\left(-\partial_{y} u \partial_{x} \omega+\partial_{x} u \partial_{y} \omega\right) \partial_{x}^{j-k} u
$$

Denoting $a_{j}:=\left\|\partial_{x}^{j}\left(-\partial_{y} u \partial_{x} \omega+\partial_{x} u \partial_{y} \omega\right)\right\|_{H^{2}}$, we obtain (with (A.1), (A.2) $)^{6}$

$$
C_{3, j} \leq j^{1 / 4} \sum_{k=0}^{j / 2}\left(\begin{array}{c}
j-5 \\
k
\end{array}\right) a_{k}\|\omega\|_{\mathcal{H}_{\gamma}^{j-k}}+\sum_{k=j / 2}^{j-5}\left(\begin{array}{c}
j-5 \\
k
\end{array}\right) a_{k} \sum_{m=0}^{1}\|\omega\|_{\mathcal{H}_{\gamma}^{j-k+m}}=C_{3, j}^{1}+C_{3, j}^{2} .
$$

We get

$$
C_{3, j}^{1} \lesssim \sum_{k=0}^{j / 2}\left(\begin{array}{l}
j \\
k
\end{array}\right) a_{(k-3)+3}(j-k)^{1 / 4}\|\omega\|_{\mathcal{H}_{\gamma}^{j-k}}
$$

because $\left(\begin{array}{c}j-5 \\ k\end{array}\right) \lesssim\left(\begin{array}{l}j \\ k\end{array}\right)$ and $j^{1 / 4} \lesssim(j-k)^{1 / 4}$ for $0 \leq k \leq j / 2$. We then use Lemma 1 (with $m=3$ ) to obtain

$$
\left\|C_{3, j}^{1}\right\|_{l^{2}(\tau)} \lesssim\left\|a_{j-3}\right\|_{l^{2}(\tau)}\left\|j^{1 / 4}\right\| \omega\left\|_{\mathcal{H}_{\gamma}^{j}}\right\|_{l^{2}(\tau)}
$$


Then, we write

$$
\begin{aligned}
C_{3, j}^{2} & =j^{1 / 4} \sum_{k=j / 2+3}^{j-2}\left(\begin{array}{l}
j-5 \\
k-3
\end{array}\right) a_{k-3} \sum_{m=3}^{4}\|\omega\|_{\mathcal{H}_{\gamma}^{j-k+m}} \\
& \lesssim \sum_{k=j / 2}^{j}\left(\begin{array}{l}
j \\
k
\end{array}\right) k^{1 / 4} a_{k-3} \sum_{m=3}^{4}\|\omega\|_{\mathcal{H}_{\gamma}^{j-k}} .
\end{aligned}
$$

because $\left(\begin{array}{l}j-5 \\ k-3\end{array}\right) \lesssim\left(\begin{array}{l}j \\ k\end{array}\right)$ and $j^{1 / 4} \lesssim k^{1 / 4}$ for $j / 2 \leq k \leq j$. Hence,

$$
\left\|C_{3, j}^{2}\right\|_{l^{2}(\tau)} \lesssim\left\|j^{1 / 4} a_{j-3}\right\|_{l^{2}(\tau)}\|\| \omega\left\|_{\mathcal{H}_{\gamma}^{j}}\right\|_{l^{2}(\tau)} \lesssim\left\|j^{1 / 4} a_{j-3}\right\|_{l^{2}(\tau)}
$$

Finally, applying the same type of arguments, it is shown that

$\left\|a_{j-3}\right\|_{l^{2}(\tau)} \lesssim\|\| \omega\left\|_{\mathcal{H}_{\gamma}^{j}}\right\|_{l^{2}(\tau)} \lesssim \sqrt{E_{\omega}(t, \tau)}, \quad\left\|j^{1 / 4} a_{j-3}\right\|_{l^{2}(\tau)} \lesssim\left\|j^{1 / 4}\right\| \omega\left\|_{\mathcal{H}_{\gamma}^{j}}\right\|_{l^{2}(\tau)} \lesssim \sqrt{\partial_{\tau} E_{\omega}(t, \tau)}$

Inserting these bounds into (3.65)-(3.66) and summing:

$$
\left\|C_{3, j}\right\|_{l^{2}(\tau)} \lesssim \sqrt{E_{\omega}(t, \tau)}
$$

\section{Estimate on $D_{j}$}

We now turn to the most delicate term

$$
\begin{aligned}
D_{j}= & -2 j^{3 / 2} \int_{\mathbb{T} \times \mathbb{R}_{+}} \partial_{x}^{j-5} \mathcal{C}_{5} \tilde{g}_{j}=-2 j^{3 / 2} \int_{\mathbb{T} \times \mathbb{R}_{+}} \partial_{y} \omega \partial_{x}^{j} \partial_{y} \omega \tilde{g}_{j} \\
& -2 j^{3 / 2} \int_{\mathbb{T} \times \mathbb{R}_{+}}\left[\partial_{x}^{j-5}, \partial_{y} \omega\right] \partial_{y} \partial_{x}^{5} \omega \tilde{g}_{j}:=D_{j}^{1}+D_{j}^{2}
\end{aligned}
$$

Study of $D_{j}^{2}$. We integrate by parts to find

$$
D_{j}^{2}=2 j^{3 / 2} \int_{\mathbb{T} \times \mathbb{R}_{+}}\left[\partial_{x}^{j-5}, \partial_{y} \omega\right] \partial_{x}^{5} \omega \partial_{y} \tilde{g}_{j}+2 j^{3 / 2} \int_{\mathbb{T} \times \mathbb{R}_{+}}\left[\partial_{x}^{j-5}, \partial_{y}^{2} \omega\right] \partial_{x}^{5} \omega \tilde{g}_{j}:=D_{j}^{2^{\prime}}+D_{j}^{2^{\prime \prime}}
$$

Note that there is no boundary term, as $\left.\left(\partial_{x}^{k} \partial_{y} \omega\right)\right|_{y=0}=\left.\partial_{x}^{k} \partial_{y}^{2} u\right|_{y=0}=0$ for any $k$, using the Prandtl equation. We have by Cauchy-Schwarz inequality :

$$
\left\|D_{j}^{2^{\prime}}\right\|_{l^{1}(\tau)} \leq\left\|j^{3 / 4}\right\|\left[\partial_{x}^{j-5}, \partial_{y} \omega\right] \partial_{x}^{5} \omega\left\|_{L^{2}\left(\mathbb{T} \times \mathbb{R}_{+}\right)}\right\|_{l^{2}(\tau)}\left\|j^{3 / 4}\right\| \partial_{y} \tilde{g}_{j}\left\|_{L^{2}\left(\mathbb{T} \times \mathbb{R}_{+}\right)}\right\|_{l^{2}(\tau)} .
$$

We find, by now standard arguments:

$$
\left\|j^{3 / 4}\right\|\left[\partial_{x}^{j-5}, \partial_{y} \omega\right] \partial_{x}^{5} \omega\left\|_{L^{2}\left(\mathbb{T} \times \mathbb{R}_{+}\right)}\right\|_{l^{2}(\tau)} \lesssim\left\|j^{3 / 4} j\right\| \omega\left\|_{\mathcal{H}_{\gamma}^{j-1}}\right\|_{l^{2}(\tau)}
$$

We notice that $\left\|j^{7 / 4}\right\| \omega\left\|_{\mathcal{H}_{\gamma}^{j-1}}\right\|_{l^{2}(\tau)} \lesssim\|\| \omega\left\|_{\mathcal{H}_{\gamma}^{j}}\right\|_{l^{2}(\tau)}$ to conclude that

$$
\left\|D_{j}^{2^{\prime}}\right\|_{l^{1}(\tau)} \lesssim \sqrt{E_{\omega}(t, \tau)} \sqrt{D_{g}^{2}(t, \tau)} \lesssim \eta D_{g}^{2}(t, \tau)+C_{\eta} E_{\omega}(t, \tau)
$$

The treatment of $D_{j}^{2^{\prime \prime}}$ is similar: we state

$$
\left\|D_{j}^{2^{\prime \prime}}\right\|_{l^{1}(\tau)} \lesssim \sqrt{E_{\omega}(t, \tau)} \sqrt{E_{g}^{2}(t, \tau)} \lesssim E_{g}^{2}(t, \tau)+E_{\omega}(t, \tau)
$$


Finally,

$$
\left\|D_{j}^{2}\right\|_{l^{1}(\tau)} \lesssim \eta D_{g}^{2}(t, \tau)+C_{\eta} E_{\omega}(t, \tau)+E_{g}^{2}(t, \tau)
$$

Study of $D_{j}^{1}$. We shall use the decomposition of $\partial_{x}^{j} u$ recalled in (3.37):

$$
\partial_{x}^{j} u=C_{j} 1_{\{y>a\}} \omega+u_{g}^{j} .
$$

Let $\varepsilon>0, \chi^{\varepsilon, a} \in C_{c}^{\infty}(\mathbb{R})$, satisfying

$$
\chi^{\varepsilon, a}=1 \quad \text { over }\left\{|y-a| \leq \frac{\varepsilon}{2}\right\}, \quad \chi^{\varepsilon, a}=0 \quad \text { outside }\{|y-a| \geq \varepsilon\} .
$$

We shall keep track of the $\varepsilon$-dependence in all inequalities below. In particular, notation $f \lesssim g$ will always refer to an inequality $f \leq C g$ for a constant $C$ that does not depend on $\varepsilon$. We write

$$
D_{j}^{1}=-2 j^{3 / 2} \int_{\mathbb{T} \times \mathbb{R}_{+}} \chi^{\varepsilon, a} \partial_{y} \omega \partial_{x}^{j} \partial_{y} \omega \tilde{g}_{j}-2 j^{3 / 2} \int_{\mathbb{T} \times \mathbb{R}_{+}}\left(1-\chi^{\varepsilon, a}\right) \partial_{y} \omega \partial_{x}^{j} \partial_{y} \omega \tilde{g}_{j}:=D_{j}^{1^{\prime}}+D_{j}^{1^{\prime \prime}} .
$$

As $\omega$ does not vanish away from $y=a$, we can write

$$
\begin{aligned}
& D_{j}^{1^{\prime \prime}}=-2 j^{3 / 2} \int_{\mathbb{T} \times \mathbb{R}_{+}}\left(1-\chi^{\varepsilon, a}\right) \frac{\partial_{y} \omega}{\omega}\left(\partial_{y} g_{j}-\partial_{y}^{2} \omega \partial_{x}^{j} u\right) \tilde{g}_{j}=2 j^{3 / 2} \int_{\mathbb{T} \times \mathbb{R}_{+}} \partial_{y}\left(\left(1-\chi^{\varepsilon, a}\right) \frac{\partial_{y} \omega}{\omega}\right) g_{j} \tilde{g}_{j} \\
& +2 j^{3 / 2} \int_{\mathbb{T} \times \mathbb{R}_{+}}\left(1-\chi^{\varepsilon, a}\right) \frac{\partial_{y} \omega}{\omega} g_{j} \partial_{y} \tilde{g}_{j}+2 j^{3 / 2} \int_{\mathbb{T} \times \mathbb{R}_{+}}\left(1-\chi^{\varepsilon, a}\right) \frac{\partial_{y} \omega}{\omega} \partial_{y}^{2} \omega \partial_{x}^{j} u \tilde{g}_{j}
\end{aligned}
$$

It follows that

$$
\left\|D_{j}^{1^{\prime \prime}}\right\|_{l^{1}(\tau)} \lesssim C_{\varepsilon}\left\|j^{3 / 4}\right\| g_{j}\left\|_{L^{2}}\right\|_{l^{2}(\tau)}\left\|j^{3 / 4}\right\| \partial_{y} \tilde{g}_{j}\left\|_{L^{2}}\right\|_{l^{2}(\tau)}+\left\|j^{1 / 4}\right\| \omega\left\|_{\mathcal{H}_{\gamma}^{j}}\right\|_{l^{2}(\tau)}\left\|j^{5 / 4}\right\| \tilde{g}_{j}\left\|_{L^{2}}\right\|_{l^{2}(\tau)}
$$

Pondering on Lemma 7, we get

$$
\begin{aligned}
\left\|D_{j}^{1^{\prime \prime}}\right\|_{l^{1}(\tau)} & \lesssim C_{\varepsilon}\left(\sqrt{E_{g}^{2}(t, \tau)}+\sqrt{E_{\omega}(t, \tau)}\right) \sqrt{D_{g}^{2}(t, \tau)}+C_{\varepsilon} \sqrt{\partial_{\tau} E_{\omega}(t, \tau)} \sqrt{\partial_{\tau} E_{g}^{2}(t, \tau)} \\
& \lesssim \eta D_{g}^{2}(t, \tau)+C_{\eta}\left(\partial_{\tau} E_{\omega}(t, \tau)+\partial_{\tau} E_{g}^{2}(t, \tau)\right)
\end{aligned}
$$

It remains to handle $D_{j}^{1^{\prime}}$. Therefore, we introduce

$$
\omega_{h}^{j}:=1_{y>a} C_{j} \partial_{y} \omega, \quad \omega_{g}^{j}:=\partial_{y} u_{g}^{j}
$$

We emphasize that both $\omega_{h}^{j}$ and $\omega_{g}^{j}$ are discontinuous across $y=a$, contrary to $\partial_{x}^{j} \omega=\omega_{h}^{j}+\omega_{g}^{j}$. We find

$$
\begin{aligned}
D_{j}^{1^{\prime}}= & -2 j^{3 / 2} \int_{\{y>a\}} \chi^{\varepsilon, a} \partial_{y} \omega \partial_{y} \omega_{h}^{j} \tilde{g}_{j}-2 j^{3 / 2} \int_{\{y>a\}} \chi^{\varepsilon, a} \partial_{y} \omega \partial_{y} \omega_{g}^{j} \tilde{g}_{j}-2 j^{3 / 2} \int_{\{y<a\}} \chi^{\varepsilon, a} \partial_{y} \omega \partial_{y} \omega_{g}^{j} \tilde{g}_{j} \\
= & -2 j^{3 / 2} \int_{\{y>a\}} \chi^{\varepsilon, a} \partial_{y} \omega \partial_{y} \omega_{h}^{j} \tilde{g}_{j}+2 j^{3 / 2} \int_{\mathbb{T} \times \mathbb{R}_{+}} \partial_{y}\left(\chi^{\varepsilon, a} \partial_{y} \omega\right) \omega_{g}^{j} \tilde{g}_{j}+2 j^{3 / 2} \int_{\mathbb{T} \times \mathbb{R}_{+}} \chi^{\varepsilon, a} \partial_{y} \omega \omega_{g}^{j} \partial_{y} \tilde{g}_{j} \\
& +2 j^{3 / 2} \int_{\{y=a\}} \partial_{y} \omega\left[\omega_{g}^{j}\right] \tilde{g}_{j}:=I+I I+I I I+I V
\end{aligned}
$$


To go from the first to the second equality, we have integrated by parts the last two integrals: gathering the boundary terms at $y=a^{+}$and $y=a^{-}$we have obtained a jump term

$$
\left[\omega_{g}^{j}\right]:=\left.\omega_{g}^{j}\right|_{y=a^{+}}-\left.\omega_{g}^{j}\right|_{y=a^{-}}
$$

across $y=a$. Clearly,

$$
\begin{aligned}
\|I\|_{l^{1}(\tau)} & \lesssim\left\|j^{1 / 4}\right\| C_{j}\left\|_{L^{2}(\mathbb{T})}\right\|_{l^{2}(\tau)}\left\|j^{5 / 4}\right\| \tilde{g}_{j}\left\|_{L^{2}\left(\mathbb{T} \times \mathbb{R}_{+}\right.}\right\|_{l^{2}(\tau)} \lesssim \sqrt{\partial_{\tau} E_{\omega}(t, \tau)} \sqrt{\partial_{\tau} E_{g}^{2}(t, \tau)} \\
& \lesssim \partial_{\tau} E_{\omega}(t, \tau)+\partial_{\tau} E_{g}^{2}(t, \tau)
\end{aligned}
$$

with the last inequality coming from (3.25). Also

$$
\begin{aligned}
\|I I\|_{l^{1}(\tau)} & \lesssim\left\|j^{1 / 4}\right\| \omega_{g}^{j}\left\|_{L^{2}\left(\mathbb{T} \times \mathbb{R}_{+}\right)}\right\|_{l^{2}(\tau)}\left\|j^{5 / 4}\right\| \tilde{g}_{j}\left\|_{L^{2}\left(\mathbb{T} \times \mathbb{R}_{+}\right.}\right\|_{l^{2}(\tau)} \\
& \lesssim\left(\left\|j^{1 / 4}\right\| C_{j}\left\|_{L^{2}(\mathbb{T})}\right\|_{l^{2}(\tau)}+\left\|j^{1 / 4}\right\| \partial_{x}^{j} \omega\left\|_{L^{2}\left(\mathbb{T} \times \mathbb{R}_{+}\right)}\right\|_{l^{2}(\tau)}\right)\left\|j^{5 / 4}\right\| \tilde{g}_{j}\left\|_{L^{2}\left(\mathbb{T} \times \mathbb{R}_{+}\right)}\right\|_{l^{2}(\tau)} \\
& \lesssim \sqrt{\partial_{\tau} E_{\omega}(t, \tau)} \sqrt{\partial_{\tau} E_{g}^{2}(t, \tau)} \lesssim \partial_{\tau} E_{\omega}(t, \tau)+\partial_{\tau} E_{g}^{2}(t, \tau)
\end{aligned}
$$

As regards $I V$, we have

$$
I V=-2 j^{3 / 2} \int_{\{y=a\}} \partial_{y} \omega\left[\omega_{h}^{j}\right] \tilde{g}_{j}=-2 j^{3 / 2} \int_{\{y=a\}}\left(\partial_{y} \omega\right)^{2} C_{j} \tilde{g}_{j}
$$

so that

$$
|I V| \lesssim\left\|j^{1 / 2} C_{j}\right\|_{L^{2}(\mathbb{T})}\left\|j^{5 / 4} \tilde{g}_{j}\right\|_{L^{2}\left(\mathbb{T} \times \mathbb{R}_{+}\right)}^{1 / 2}\left\|j^{3 / 4} \partial_{y} \tilde{g}_{j}\right\|_{L^{2}\left(\mathbb{T} \times \mathbb{R}_{+}\right)}^{1 / 2}
$$

and finally

$$
\|I V\|_{l^{1}(\tau)} \lesssim \sqrt{\partial_{\tau} E_{\omega}(t, \tau)} E_{g}^{2}(t, \tau)^{1 / 4} D_{g}^{2}(t, \tau)^{1 / 4} \lesssim \eta D_{g}^{2}(t, \tau)+C_{\eta}\left(\partial_{\tau} E_{\omega}(t, \tau)+E_{g}^{2}(t, \tau)\right)
$$

To handle $I I I$, we split the integral as follows:

$$
\int_{\mathbb{T} \times \mathbb{R}_{+}} \chi^{\varepsilon, a} \partial_{y} \omega \omega_{g}^{j} \partial_{y} \tilde{g}_{j}=\int_{\mathbb{T} \times \mathbb{R}_{+}} \chi^{\varepsilon, a} \partial_{y} \omega \omega_{g}^{j} \partial_{y} g_{j}+\int_{\mathbb{T} \times \mathbb{R}_{+}} \chi^{\varepsilon, a} \partial_{y} \omega \omega_{g}^{j} \partial_{y}\left(\tilde{g}_{j}-g_{j}\right)=i+i i
$$

Note that for $\varepsilon$ small enough, on the support of $\chi^{\varepsilon, a}$, one has:

$$
g_{j}=\omega \partial_{x}^{j} \omega-\partial_{y} \omega \partial_{x}^{j} u=\omega \omega_{g}^{j}-\partial_{y} \omega u_{g}^{j} .
$$

so that

$$
\partial_{y} g_{j}=\omega \partial_{y} \omega_{g}^{j}-\partial_{y}^{2} \omega u_{g}^{j}
$$

We write

$$
\begin{aligned}
i= & \int_{\mathbb{T} \times \mathbb{R}_{+}} \chi^{\varepsilon, a} \partial_{y} \omega \omega \frac{1}{2} \partial_{y}\left(\omega_{g}^{j}\right)^{2}-\int_{\mathbb{T} \times \mathbb{R}_{+}} \chi^{\varepsilon, a} \partial_{y} \omega \partial_{y}^{2} \omega \omega_{g}^{j} u_{g}^{j} \\
= & -\frac{1}{2} \int_{\mathbb{T} \times \mathbb{R}_{+}} \chi^{\varepsilon, a}\left(\partial_{y} \omega\right)^{2}\left(\omega_{g}^{j}\right)^{2}-\frac{1}{2} \int_{\mathbb{T} \times \mathbb{R}_{+}} \partial_{y} \chi^{\varepsilon, a} \partial_{y} \omega \omega\left(\omega_{g}^{j}\right)^{2}-\frac{1}{2} \int_{\mathbb{T} \times \mathbb{R}_{+}} \chi^{\varepsilon, a} \partial_{y}^{2} \omega \omega\left(\omega_{g}^{j}\right)^{2} \\
& +\int_{\mathbb{T} \times \mathbb{R}_{+}} \chi^{\varepsilon, a} \partial_{y}^{2} \omega \omega_{g}^{j}\left(g_{j}-\omega \omega_{g}^{j}\right)
\end{aligned}
$$


Note that, by assumption (3.1), one has for $\varepsilon>0$ small enough:

$$
-\frac{1}{2} \int_{\mathbb{T} \times \mathbb{R}_{+}} \chi^{\varepsilon, a}\left(\partial_{y} \omega\right)^{2}\left(\omega_{g}^{j}\right)^{2} \leq-\eta \int_{\mathbb{T} \times \mathbb{R}_{+}} \chi^{\varepsilon, a}\left(\omega_{g}^{j}\right)^{2}
$$

where

$$
2 \eta:=\frac{1}{2} \inf _{t, x}\left(\partial_{y} \omega(t, a(t, x))\right)^{2} .
$$

On the other hand, as $\omega$ vanishes linearly in $y-a$ at the critical curve:

$$
\frac{1}{2}\left|\int_{\mathbb{T} \times \mathbb{R}_{+}} \chi^{\varepsilon, a} \partial_{y}^{2} \omega \omega\left(\omega_{g}^{j}\right)^{2}\right| \lesssim \varepsilon \int_{\mathbb{T} \times \mathbb{R}_{+}} \chi^{\varepsilon, a}\left(\omega_{g}^{j}\right)^{2}
$$

For $\varepsilon$ small enough, we can absorb the latter by the former, resulting in

$$
i \leq-\frac{\eta}{2} \int_{\mathbb{T} \times \mathbb{R}_{+}} \chi^{\varepsilon, a}\left(\omega_{g}^{j}\right)^{2}-\frac{1}{2} \int_{\mathbb{T} \times \mathbb{R}_{+}} \partial_{y} \chi^{\varepsilon, a} \partial_{y} \omega \omega\left(\omega_{g}^{j}\right)^{2}+\int_{\mathbb{T} \times \mathbb{R}_{+}} \chi^{\varepsilon, a} \partial_{y}^{2} \omega \omega_{g}^{j} g_{j}
$$

Note that $\partial_{y} \chi^{\varepsilon, a}$ vanishes in a neighborhood of $y=a$ so that, roughly speaking, $\omega_{g}^{j}$ behaves like $g_{j}$ over the support of $\partial_{y} \chi^{\varepsilon, a}$. More precisely,

$$
\left|\int_{\mathbb{T} \times \mathbb{R}_{+}} \partial_{y} \chi^{\varepsilon, a} \partial_{y} \omega \omega\left(\omega_{g}^{j}\right)^{2}\right| \leq C_{\varepsilon}\left\|g_{j}\right\|_{L^{2}\left(\mathbb{T} \times \mathbb{R}_{+}\right)}^{2}
$$

Finally, we apply a Young's inequality to the last term:

$$
\left|\int_{\mathbb{T} \times \mathbb{R}_{+}} \chi^{\varepsilon, a} \partial_{y}^{2} \omega \omega_{g}^{j} g_{j}\right| \leq \frac{\eta}{4} \int_{\mathbb{T} \times \mathbb{R}_{+}} \chi^{\varepsilon, a}\left(\omega_{g}^{j}\right)^{2}+C_{\eta}\left\|g_{j}\right\|_{L^{2}\left(\mathbb{T} \times \mathbb{R}_{+}\right)}^{2} .
$$

We obtain, for $\eta>0$ small enough

$$
i \lesssim-\eta \int_{\mathbb{T} \times \mathbb{R}_{+}} \chi^{\varepsilon, a}\left(\omega_{g}^{j}\right)^{2}+\left\|g_{j}\right\|_{L^{2}\left(\mathbb{T} \times \mathbb{R}_{+}\right)}^{2}
$$

Finally, we apply Young's inequality to $i i$ : for all $\eta^{\prime}>0$,

$$
i i \lesssim \eta^{\prime} \int_{\mathbb{T} \times \mathbb{R}_{+}} \chi^{\varepsilon, a}\left(\omega_{g}^{j}\right)^{2}+C_{\eta^{\prime}}\left\|\sqrt{\chi^{\varepsilon, a}} \partial_{y}\left(\tilde{g}_{j}-g_{j}\right)\right\|_{L^{2}\left(\mathbb{T} \times \mathbb{R}_{+}\right)}^{2}
$$

Combining with the bound on $i$, we find (for $\eta^{\prime}$ small enough)

$$
\max (I I I, 0)=2 j^{3 / 2} \max (i+i i, 0) \lesssim j^{3 / 2}\left\|g_{j}\right\|_{L^{2}\left(\mathbb{T} \times \mathbb{R}_{+}\right)}^{2}+j^{3 / 2}\left\|\sqrt{\chi^{\varepsilon, a}} \partial_{y}\left(\tilde{g}_{j}-g_{j}\right)\right\|_{L^{2}\left(\mathbb{T} \times \mathbb{R}_{+}\right)}^{2}
$$

If we gather this last bound with those on $I, I I$ and $I V$, we conclude that

$$
\begin{aligned}
\left\|\max \left(D_{j}^{1^{\prime}}, 0\right)\right\|_{l^{1}(\tau)} \lesssim & \eta D_{g}^{2}(t, \tau)+C_{\eta}\left(\partial_{\tau} E_{\omega}(t, \tau)+\partial_{\tau} E_{g}^{2}(t, \tau)\right) \\
& +\left\|j^{3 / 4}\right\| g_{j}\left\|_{L^{2}\left(\mathbb{T} \times \mathbb{R}_{+}\right)}\right\|_{l^{2}(\tau)}^{2}+\left\|j^{3 / 4}\right\| \sqrt{\chi^{\varepsilon, a}} \partial_{y}\left(\tilde{g}_{j}-g_{j}\right)\left\|_{L^{2}\left(\mathbb{T} \times \mathbb{R}_{+}\right)}\right\|_{l^{2}(\tau)}^{2}
\end{aligned}
$$

We then use Lemma 7 together with

\section{Lemma 9}

$$
\left\|j^{3 / 4}\right\| \sqrt{\chi^{\varepsilon, a}} \partial_{y}\left(\tilde{g}_{j}-g_{j}\right)\left\|_{L^{2}\left(\mathbb{T} \times \mathbb{R}_{+}\right)}\right\|_{l^{2}(\tau)}^{2} \lesssim D_{h}(t, \tau)+E_{\omega}(t, \tau) .
$$


This last inequality will be proved in appendix . We obtain

$$
\left\|\max \left(D_{j}^{1^{\prime}}, 0\right)\right\|_{l^{1}(\tau)} \lesssim \eta D_{g}^{2}(t, \tau)+D_{h}(t, \tau)+C_{\eta}\left(\partial_{\tau} E_{\omega}(t, \tau)+\partial_{\tau} E_{g}^{2}(t, \tau)\right)
$$

In turn, we can combine this bound with those on $D_{j}^{1^{\prime \prime}}, D_{j}^{2}$, to obtain

$$
\left\|\max \left(D_{j}, 0\right)\right\|_{l^{1}(\tau)} \lesssim \eta D_{g}^{2}(t, \tau)+D_{h}(t, \tau)+C_{\eta}\left(\partial_{\tau} E_{\omega}(t, \tau)+\partial_{\tau} E_{g}^{2}(t, \tau)\right)
$$

Then, we collect the estimates on $A_{j}, B_{j}, C_{i, j}$. We find

$$
\begin{aligned}
\partial_{t} E_{g}^{2}(t, \tau)+D_{g}^{2}(t, \tau) & \lesssim\left(\sqrt{D_{g}^{2}(t, \tau)}+\sqrt{\partial_{\tau} E_{g}^{2}(t, \tau)}+\sqrt{\partial_{\tau} E_{\omega}(t, \tau)}\right) \sqrt{E_{g}^{2}(t, \tau)} \\
& +\eta D_{g}^{2}(t, \tau)+D_{h}(t, \tau)+C_{\eta}\left(\partial_{\tau} E_{\omega}(t, \tau)+\partial_{\tau} E_{g}^{2}(t, \tau)\right) \\
& \lesssim \eta D_{g}^{2}(t, \tau)+D_{h}(t, \tau)+C_{\eta}\left(\partial_{\tau} E_{\omega}(t, \tau)+\partial_{\tau} E_{g}^{2}(t, \tau)\right)
\end{aligned}
$$

This concludes the proof of the proposition.

\subsection{Estimate of the (first) monotonicity energy}

In the previous paragraphs, we have derived bounds on the time variations of $\dot{E}_{\omega}, E_{h}$ and $E_{g}^{2}$. These bounds involve the total energy $E_{\omega}$ defined in (2.1). By Lemma 2, this energy is controlled by the sum of $\dot{E}_{\omega}, E_{h}$ and $E_{g}^{1}$. Hence, to establish our main a priori estimate (Theorem 2), it remains to control $\partial_{t} E_{g}^{1}$. This is the purpose of

Proposition 4 Under the same assumptions as in Proposition 4, one has for some $C>0$ and all $t \in] 0, T]$ :

$$
\partial_{t} E_{g}^{1}(t, \tau)+D_{g}^{1}(t, \tau) \leq C \partial_{\tau} E_{\omega}(t, \tau)
$$

Naturally,

$$
D_{g}^{1}(t, \tau):=\sum_{j \in \mathbb{N}}\left(\tau^{j}(j !)^{-7 / 4}(j+1)^{10}\right)^{2}\left\|\partial_{y} g_{j}(t, \cdot)\right\|_{L^{2}\left(L_{\gamma}^{2}\right)}^{2}
$$

To prove this proposition, we first write the equation on

$$
g_{j}:=\tilde{\psi}\left(\partial_{x}^{j} \omega-\frac{\partial_{y} \omega}{\omega} \partial_{x}^{j} u\right), \quad \tilde{\psi}:=\psi \omega+(1-\psi) .
$$

We apply the operator $\tilde{\psi} \partial_{x}^{j}$ to the vorticity equation, apply $\tilde{\psi} \frac{\partial_{y} \omega}{\omega} \partial_{x}^{j}$ to the velocity equation, and subtract one from another. A straightforward computation gives

$$
\begin{aligned}
& \partial_{t} g_{j}+u \partial_{x} g_{j}+v \partial_{y} g_{j}-\partial_{y}^{2} g_{j} \\
& =-\sum_{k=1}^{j}\left(\begin{array}{l}
j \\
k
\end{array}\right) \partial_{x}^{k} u g_{j-k+1}-\sum_{k=1}^{j-1}\left(\begin{array}{l}
j \\
k
\end{array}\right) \partial_{x}^{k} v \tilde{\psi}\left(\partial_{y} \partial_{x}^{j-k} \omega-\frac{\partial_{y} \omega}{\omega} \partial_{x}^{j-k} \omega\right) \\
& +\left(\partial_{t}+u \partial_{x}+v \partial_{y}-\partial_{y}^{2}\right) \tilde{\psi} \partial_{x}^{j} \omega-\left(\partial_{t}+u \partial_{x}+v \partial_{y}-\partial_{y}^{2}\right)\left(\tilde{\psi} \frac{\partial_{y} \omega}{\omega}\right) \partial_{x}^{j} u \\
& -2 \partial_{y} \tilde{\psi} \partial_{y} \partial_{x}^{j} \omega+2 \partial_{y}\left(\tilde{\psi} \frac{\partial_{y} \omega}{\omega}\right) \partial_{x}^{j} \omega:=\sum_{i=1}^{6} \mathcal{C}_{i}
\end{aligned}
$$


Then, one multiplies by $\left(\tau^{j}(j !)^{-7 / 4}(j+1)^{10}\right)^{2}(1+y)^{2 \gamma} g_{j}$ and perform a standard energy estimate:

$\partial_{t} E_{g}^{1}(t, \tau)+D_{g}^{1}(t, \tau) \leq\left(\left\|A_{j}\right\|_{l^{2}(\tau)}+\left\|B_{j}\right\|_{l^{2}(\tau)}+\sum_{i \neq 5}\left\|C_{i, j}\right\|_{l^{2}(\tau)}\right) \sqrt{\partial_{\tau} E_{g}^{1}(t, \tau)}+\left\|D_{j}\right\|_{l^{1}(\tau)}$

where:

$$
\begin{aligned}
A_{j} & :=\frac{1}{j^{1 / 2}}(2 \gamma-1)\left\|(1+y)^{\gamma-1} v g_{j}\right\|_{L^{2}\left(\mathbb{T} \times \mathbb{R}_{+}\right)}, \quad B_{j}:=\frac{1}{j^{1 / 2}}(2 \gamma-1)\left\|(1+y)^{\gamma-1} \partial_{y} g_{j}\right\|_{L^{2}\left(\mathbb{T} \times \mathbb{R}_{+}\right)} \\
C_{i, j} & :=\frac{1}{j^{1 / 2}}\left\|\mathcal{C}_{i}\right\|_{L^{2}\left(L_{\gamma}^{2}\right)}, \quad i=1,2,3,4,6, \quad D_{j}:=\int_{\mathbb{T} \times \mathbb{R}_{+}} 2(1+y)^{2 \gamma} \partial_{y} \tilde{\psi} \partial_{y} \partial_{x}^{j} \omega g_{j} .
\end{aligned}
$$

Again, there is no boundary term at $y=0$, as $\partial_{y} g_{j}$ vanishes there.

To bound above terms, one can follow the calculations already performed, notably those related to Proposition 1, Note that to control the behavior at infinity of the $\mathcal{C}_{i}$ 's, one must have information on the behavior of the function $\frac{\partial_{y} \omega}{\omega}$ at infinity. Such information is provided by assumption (3.1). For the sake of brevity, we do not detail the computations. We have

$$
\left\|A_{j}\right\|_{l^{2}(\tau)} \lesssim \sqrt{E_{\omega}(t, \tau)}, \quad\left\|B_{j}\right\|_{l^{2}(\tau)} \lesssim \sqrt{D_{g}^{2}(t, \tau)}
$$

As regards the nonlinear terms, we have

$$
\left\|C_{i, j}\right\|_{l^{2}(\tau)} \lesssim \sqrt{E_{\omega}(t, \tau)} \sqrt{\partial_{\tau} E_{\omega}(t, \tau)} \lesssim \sqrt{\partial_{\tau} E_{\omega}(t, \tau)}, \quad i=1,2
$$

whereas

$$
\left\|C_{i, j}\right\|_{l^{2}(\tau)} \lesssim E_{\omega}(t, \tau), \quad i=3,4,6
$$

Finally, after integration by parts, we find

$$
\left\|D_{j}\right\|_{l^{1}(\tau)} \leq C \sqrt{E_{\omega}(t, \tau)}\left(\sqrt{E_{g}^{1}(t, \tau)}+\sqrt{D_{g}^{1}(t, \tau)}\right) \lesssim \eta D_{g}^{1}(t, \tau)+C_{\eta} E_{\omega}(t, \tau), \quad \forall \eta>0
$$

Inserting all these bounds in (3.71) (and taking parameter $\eta$ small enough) yields the estimate in Proposition 4.

\section{Conclusion: Main a priori estimate}

On one hand, from propositions 1, 2 and 4, we infer that

$$
\begin{aligned}
\partial_{t}\left(\dot{E}_{\omega}+E_{h}+E_{g}^{1}\right)(t, \tau)+\left(\dot{D}_{\omega}+D_{h}+D_{g}^{1}\right)(t, \tau) & \lesssim \partial_{\tau} E_{\omega}(t, \tau)+\partial_{\tau} E_{g}^{2}(t, \tau) \\
& \lesssim \partial_{\tau}\left(\dot{E}_{\omega}+E_{h}+E_{g}^{1}\right)(t, \tau)+\partial_{\tau} E_{g}^{2}(t, \tau),
\end{aligned}
$$

where we have used (3.4) to go from the first to the second line. On the other hand, Proposition 3 yields (still with relation (3.4)):

$$
\partial_{t} E_{g}^{2}(t, \tau)+D_{g}^{2}(t, \tau) \lesssim \partial_{\tau}\left(\dot{E}_{\omega}+E_{h}+E_{g}^{1}\right)(t, \tau)+\partial_{\tau} E_{g}^{2}(t, \tau)+D_{h}(t, \tau) .
$$


Thus, we can multiply the last equation by a small factor $\alpha$, and add it to the first equation, to end up with

$$
\begin{aligned}
\partial_{t}\left(\dot{E}_{\omega}+E_{h}+E_{g}^{1}+\alpha E_{g}^{2}\right)(t, \tau)+\left(\dot{D}_{\omega}+D_{h}+D_{g}^{1}\right. & \left.+D_{g}^{2}\right)(t, \tau) \\
& \lesssim \partial_{\tau}\left(\dot{E}_{\omega}+E_{h}+E_{g}^{1}+\alpha E_{g}^{2}\right)(t, \tau)
\end{aligned}
$$

This concludes the proof of Theorem 2. We insist that the $\alpha$ depends on $M$, but can be (and in fact needs to be) taken small. In particular, we insist that we can choose $\alpha \leq 1$, that is with an upper bound independent of $M$. This will be crucial in the effective construction of solutions.

\section{Existence of a Gevrey solution}

We deal here with the existence part of Theorem 1. The core of the argument is the energy estimate stated in Theorem 2, Actually, such estimate is derived on a sequence of approximations of the Prandtl system, that we now present.

\subsection{Approximate system}

We start with an initial data satisfying

$$
u_{0} \in G_{\tau_{0}}^{7 / 4}\left(\mathbb{T} ; H_{\gamma-1}^{s+1}\right), \quad \omega_{0}:=\partial_{y} u_{0} \in G_{\tau_{0}}^{7 / 4}\left(\mathbb{T} ; H_{\gamma}^{s}\right),
$$

as well as assumptions $(\mathrm{H})$ and $\left(\mathrm{H}^{\prime}\right)$, see section 2, Parameters $\tau_{0}, s, \sigma, \delta$ are as in Theorem 1, To fix ideas, we assume that the curve of non-degenerate critical points satisfies the constraint $0<a_{0}<3$. The choice of the value 3 is of course arbitrary.

To construct solutions with all the a priori bounds of the previous section, we need to find a good approximating scheme: it should not destroy the energy estimates, and should guarantee that the point-wise bounds (3.1) (which echo those in $\left(\mathrm{H}^{\prime}\right)$ ) are preserved in small time, uniformly with respect to the approximation parameters.

The same scheme as in [17, section 4] works here. It goes through the following approximate system (regularized Prandtl system)

$$
\left\{\begin{array}{r}
\partial_{t} u^{\varepsilon}+u^{\varepsilon} \partial_{x} u^{\varepsilon}+v^{\varepsilon} \partial_{y} u^{\varepsilon}-\partial_{y}^{2} u^{\varepsilon}-\varepsilon \partial_{x}^{2} u^{\varepsilon}=0 \\
\partial_{x} u^{\varepsilon}+\partial_{y} v^{\varepsilon}=0 \\
\left.u^{\varepsilon}\right|_{y=0}=\left.v^{\varepsilon}\right|_{y=0}=0, \lim _{y=+\infty} u^{\varepsilon}=0 .
\end{array}\right.
$$

The big difference with the original Prandtl system is the tangential diffusion $-\varepsilon \partial_{x}^{2} u$, which allows to control the loss of $x$-derivative generated by the $v$-term. It restores well-posedness in the Sobolev setting. A detailed construction of solutions of (4.2) is performed in [17]. Let us denote

$$
H^{s, \gamma}\left(\mathbb{T} \times \mathbb{R}_{+}\right):=\cap_{k=0}^{s} H_{x}^{k}\left(\mathbb{T}, H_{\gamma}^{s-k}\left(\mathbb{R}_{+}\right)\right) .
$$

As a result of [17, for any initial data

$$
u_{0} \in H^{s, \gamma-1}\left(\mathbb{T} \times \mathbb{R}_{+}\right), \quad \omega_{0}:=\partial_{y} u_{0} \in H^{s, \gamma}\left(\mathbb{T} \times \mathbb{R}_{+}\right)
$$


there is a unique $T_{*}^{\varepsilon} \in \mathbb{R}_{+}^{*} \cup\{+\infty\}$, and a unique maximal solution

$$
u^{\varepsilon} \in L_{l o c}^{\infty}\left(\left[0, T_{*}^{\varepsilon}\right) ; H^{s, \gamma-1}\left(\mathbb{T} \times \mathbb{R}_{+}\right)\right), \quad \omega^{\varepsilon}:=\partial_{y} u^{\varepsilon} \in L_{l o c}^{2}\left(\left[0, T_{*}^{\varepsilon}\right) ; H^{s, \gamma}\left(\mathbb{T} \times \mathbb{R}_{+}\right)\right) .
$$

of (4.2). By maximal, we mean that

$$
\text { either } T_{*}^{\varepsilon}=+\infty, \quad \text { or } \quad \limsup _{t \rightarrow T_{*}^{\varepsilon}}\left\|\omega^{\varepsilon}(t)\right\|_{H^{s, \gamma}\left(\mathbb{T} \times \mathbb{R}_{+}\right)}=+\infty
$$

Of course, our Gevrey data $u_{0}$ is regular enough to apply this result. Furthermore, up to some small time (possibly depending on $\varepsilon$ ), the solution $u^{\varepsilon}\left(\right.$ resp. $\left.\omega^{\varepsilon}\right)$ remains in $G_{\tilde{\tau}_{0}}^{7 / 4}\left(H_{\gamma-1}^{s+1}\right)$ (resp. $\left.G_{\tilde{\tau}_{0}}^{7 / 4}\left(H_{\gamma}^{s}\right)\right)$, for any $\tilde{\tau}_{0}<\tau_{0}$.

The fact that system (4.2) preserves Gevrey regularity in small time is somehow classical. One way to show it is to establish Gevrey bounds on $u^{\varepsilon, n}$, solution of the Galerkin type approximation

$$
\left\{\begin{aligned}
\partial_{t} u^{\varepsilon, n}+P^{n}\left(u^{\varepsilon, n} \partial_{x} u^{\varepsilon, n}+v^{\varepsilon, n} \partial_{y} u^{\varepsilon, n}\right)-\partial_{y}^{2} u^{\varepsilon, n}-\varepsilon \partial_{x}^{2} u^{\varepsilon, n}=0 \\
\partial_{x} u^{\varepsilon, n}+\partial_{y} v^{\varepsilon, n}=0, \\
\left.u^{\varepsilon, n}\right|_{y=0}=\left.v^{\varepsilon, n}\right|_{y=0}=0, \lim _{y=+\infty} u^{\varepsilon, n}=0 .
\end{aligned}\right.
$$

$P_{n}$ is the projection over the Fourier modes $|k| \leq n$ in variable $x$. One can show for this system uniform (in $n$ ) Gevrey bounds for small time $T^{\varepsilon}$ (independent of $n$ ). As $n$ goes to infinity, we get Gevrey bounds for the (unique) solution $u^{\varepsilon}$ of (4.2). We insist that these estimates are much simpler than those of the previous section. No special structure or tricky norm is needed: as the system is fully parabolic, there is no loss of $x$-derivative. For the sake of brevity, we do not give further details.

Now, let $M \geq M_{0}$ large enough, and $t \mapsto \tau(t) \in\left[\frac{\tilde{\tau}_{0}}{2}, \tilde{\tau}_{0}\right]$ a function over $\mathbb{R}$. We denote

$$
T^{\varepsilon}(\tau):=\sup \left\{T \in \left[0, T_{*}^{\varepsilon}\left[, \quad E_{\omega}(t, \tau(t)) \leq M \text { for all } t \in[0, T]\right\},\right.\right.
$$

where $E_{\omega}$ is defined in (2.1), replacing $u$ by $\left.u^{\varepsilon}\right)$. By standard $\operatorname{arguments,} T^{\varepsilon}(\tau)>0$, and $\lim \sup _{t \rightarrow T^{\varepsilon}(\tau)} E_{\omega}(t, \tau(t))=M$.

The point is to show that for a good choice of $M$ and $\tau$, there exists $T^{0}>0$ independent of $\varepsilon$, such that $T^{\varepsilon}(\tau) \geq T^{0}$. Then, the solution $u^{\varepsilon}$ will exist and have uniform Gevrey bounds on a time independent of $\varepsilon$. Finally, standard compactness arguments will allow to let $\varepsilon \rightarrow 0$, and obtain a solution of the Prandtl equation with initial data $u_{0}$ at the limit.

We now give a few hints on how to prove the inequality $T^{\varepsilon}(\tau) \geq T^{0}$. Let $M \geq M_{0}$. The key-point is to establish the following: there exists $T_{0}>0$, depending on $M$, on the initial data and on $\inf \tau=\frac{\tilde{\tau}_{0}}{2}$, but independent of $\varepsilon$, such that:

- For all $t \mapsto \tau(t) \in\left[\frac{\tilde{\tau}_{0}}{2}, \tilde{\tau}_{0}\right]$, for all $t \in\left[0, \min \left(T^{0}, T^{\varepsilon}(\tau)\right)\right]$,

$$
\left|\omega^{\varepsilon}(t, x, y)\right| \geq \frac{\delta}{(1+y)^{\sigma}}, \quad\left|\partial^{\alpha} \omega^{\varepsilon}(t, x, y)\right| \leq \frac{1}{\delta(1+y)^{\sigma+\alpha_{2}}} .
$$

for the same $\delta$ as in $\left(H^{\prime}\right)$. 
- There exists $\alpha \leq 1$, and a function $t \mapsto \tau(t) \in\left[\frac{\tilde{\tau}_{0}}{2}, \tilde{\tau}_{0}\right]$ such that:

$$
\text { For all } t \in\left[0, \min \left(T^{0}, T^{\varepsilon}(\tau)\right)\right], \quad \frac{d}{d t} \mathcal{E}(t, \tau(t)) \leq 0
$$

with

$$
\mathcal{E}(t, \tau)=\dot{E}_{\omega}(t, \tau)+E_{h}(t, \tau)+E_{g}^{1}(t, \tau)+\alpha E_{g}^{2}(t, \tau),
$$

where the energy functionals $\dot{E}_{\omega}, E_{h}(t, \tau), E_{g}^{i}$ are defined respectively in (2.8), (2.9), (2.11) and (2.13), replacing $u$ by $u^{\varepsilon}$.

We shall discuss the bounds (4.4) and (4.5) in the next paragraph. We explain first how it allows to conclude. From (4.5), we deduce that for all $t \in\left[0, \min \left(T^{0}, T^{\varepsilon}\right)\right]$,

$$
\mathcal{E}(t, \tau(t)) \leq \mathcal{E}(0, \tau(0)) .
$$

As $\alpha \in(0,1)$, this implies in turn

$\dot{E}_{\omega}(t, \tau(t))+E_{h}(t, \tau(t))+E_{g}^{1}(t, \tau(t)) \leq \dot{E}_{\omega}(0, \tau(0))+E_{h}(0, \tau(0))+E_{g}^{1}(t, \tau(0))+E_{g}^{2}(0, \tau(0))$.

By Lemma 2, we finally get

$E_{\omega}(t, \tau(t)) \leq C\left(\dot{E}_{\omega}(t, \tau(t))+E_{h}(t, \tau(t))+E_{g}^{1}(t, \tau(t))\right) \leq C\left(E_{\omega}(0, \tau(0))+E_{g}^{2}(0, \tau(0))\right)$.

A closer look at the proof of Lemma 2 shows that the constant $C$ depends on the initial data. But, up take a smaller $T_{0}$, it does not depend on the constant $M$. For instance, it depends on $\inf _{t, x \in \mathbb{T}} \partial_{y} \omega(t, a(t, x))$ : by Sobolev imbeddings and the bound $E_{\omega}(t, \tau(t)) \leq M$, one has for $T_{0}=T_{0}(M)$ small enough:

$$
\inf _{t, x \in \mathbb{T}} \partial_{y} \omega(t, a(t, x)) \geq \frac{1}{2} \inf _{x \in \mathbb{T}} \partial_{y} \omega\left(x, a_{0}(x)\right) .
$$

We leave the details to the reader. Hence, we can take

$$
M>2 C \dot{E}_{\omega}(0, \tau(0))+E_{h}(0, \tau(0))+E_{g}^{1}(t, \tau(0))+E_{g}^{2}(0, \tau(0)) .
$$

This ensures that $\mathcal{E}(t, \tau(t)) \leq \frac{M}{2}$ over $\left[0, \min \left(T^{0}, T^{\varepsilon}(\tau)\right)\right]$, and from there that $T^{\varepsilon}(\tau) \geq T^{0}$.

\subsection{Uniform bounds}

\subsubsection{Maximum principle: proof of (4.4)}

This paragraph is devoted to the proof of (4.4). One must show the upper and lower bounds over a time interval $\left[0, \min \left(T^{0}, T^{\varepsilon}\right)\right]$ for some $T^{0}$ independent of $\varepsilon$. Note that the Sobolev imbeddings and the bound $E_{\omega}(t, \tau(t)) \leq M$ allow to bound some weighted $L^{\infty}$ norms of $\omega^{\varepsilon}$ and some derivatives. However, the bounds in (4.4) can not be directly deduced from there, due to the weight $(1+y)^{\sigma}, \sigma \gg \gamma$. Nevertheless, as the regularized Prandtl equation is parabolic, it can be deduced from a maximum principle. This is described in details in article [17. The proof of the upper bounds

$$
\left|\partial^{\alpha} \omega^{\varepsilon}(t, x, y)\right| \leq \frac{1}{\delta(1+y)^{\sigma+\alpha_{2}}}, \quad \forall|\alpha| \leq 2,
$$


is exactly the same as in [17]: see inequality (5.5), and section 5.2. As regards the lower bound on $(1+y)^{\sigma} \omega^{\varepsilon}$, there is only a slight modification. In [17], $\omega \neq 0$ for all $y \geq 0$, so that the minimum principle can be applied over domains of the type $[0, T] \times \mathbb{T} \times \mathbb{R}_{+}$. In our situation, we must place ourselves above the curve of critical points. More precisely, as $\omega_{0}\left(x, a_{0}(x)\right)=$ $0, \partial_{y} \omega_{0}\left(x, a_{0}(x)\right) \neq 0$, there exists $\eta>0$, such that $\omega_{0}\left(x, a_{0}(x)+\eta\right) \neq 0$. One then applies the minimum principle over $[0, T] \times\left\{(x, y), \quad y=a_{0}(x)+\eta\right\}, T$ small enough. We refer to [17, inequality (5.6) and section 5.2.

\subsubsection{Energy estimates: proof of (4.5)}

The point here is to derive the estimates of Theorem 2, uniformly in $\varepsilon$. More precisely, one can show that for any $t \mapsto \tau(t) \in\left[\frac{\tilde{\tau}_{0}}{2}, \tilde{\tau}_{0}\right]$,

$$
\partial_{t} \mathcal{E}(t, \tau(t)) \leq C \partial_{\tau} \mathcal{E}(t, \tau(t))
$$

with a constant $C$ that does not depend on $\varepsilon$ (but depends on $M$, the initial data, and $\inf \tau=\frac{\tilde{\tau}_{0}}{2}$ ). Then,

$$
\frac{d}{d t} \mathcal{E}(\alpha, t, \tau(t))=\partial_{t} \mathcal{E}(\alpha, t, \tau(t))+\tau^{\prime}(t) \partial_{\tau} \mathcal{E}(\alpha, t, \tau(t)) \leq\left(C+\tau^{\prime}(t)\right) \mathcal{E}(\alpha, t, \tau(t)) .
$$

Then, choosing $\tau(t)=\tilde{\tau}_{0}-C t$ leads to (4.5) (up to reduce $T^{0}$ so that the constraint $\tau \geq \frac{\tilde{\tau}_{0}}{2}$ remains satisfied).

The proof of (4.6), on the approximate system (4.2), mimics the proof of the a priori estimate in Theorem 2, on the exact Prandtl system. Indeed, the additional term $-\varepsilon \partial_{x}^{2} u$ does not raise any problem. There are only two noticeable differences:

- In the energy estimate for $\dot{E}_{\omega}$, the estimate of the boundary term

$$
E_{j}:=\sum_{\substack{J=\left(j_{1}, j_{2}\right) \in \mathbb{N}^{2} \\|J|=j, 0<j_{2} \leq s}} \int_{\mathbb{T} \times\{0\}} \partial_{y} \omega_{J} \omega_{J}
$$

changes slightly. Indeed, we recall that the estimate of $E_{j}$ goes through the Lemma 4 : it uses the Prandtl equation to reduce the number of $y$ derivatives in the expression of $\left.\partial_{y}^{k} \omega\right|_{y=0}, k$ odd. Due to the regularization, the equation changes, and so the expression: the modified formula is given in [17, Lemma 5.9]. The new terms in the formula do not raise any serious difficulty. For the sake of brevity, we leave the details to the reader.

- In the energy estimate for $E_{g}^{2}$, a new commutator appears, namely

$$
2 \varepsilon \partial_{x}^{j-5}\left(\partial_{x y} \omega \partial_{x}^{6} u-2 \varepsilon \partial_{x} \omega \partial_{x}^{6} \omega\right) .
$$

It can be expanded by Leibniz rule: for brevity, we focus on one of the most difficult terms in the sum, that is:

$$
\mathcal{C}_{j}:=-2 \varepsilon \partial_{x} \omega \partial_{x}^{j+1} \omega .
$$

Proceeding as in subsection 3.4, we need to evaluate

$$
\begin{aligned}
& \left\|j^{3 / 2} \int_{\mathbb{T} \times \mathbb{R}_{+}} \mathcal{C}_{j} \tilde{g}_{j}\right\|_{l^{1}(\tau)} \\
& \lesssim \varepsilon\left\|j^{3 / 4}\right\| \partial_{x}^{j} \omega\left\|_{L^{2}}\right\|_{l^{2}(\tau)}\left\|j^{3 / 4}\right\| \partial_{x} \tilde{g}_{j}\left\|_{L^{2}}\right\|_{l^{2}(\tau)}+\varepsilon\left\|j^{1 / 4}\right\| \partial_{x}^{j} \omega\left\|_{L^{2}}\right\|_{l^{2}(\tau)}\left\|j^{5 / 4}\right\| \tilde{g}_{j}\left\|_{L^{2}}\right\|_{l^{2}(\tau)} \\
& \lesssim C_{\eta} \varepsilon\left\|j^{3 / 4}\right\| \partial_{x}^{j} \omega\left\|_{L^{2}}\right\|_{l^{2}(\tau)}^{2}+\eta \varepsilon\left\|j^{3 / 4}\right\| \partial_{x} \tilde{g}_{j}\left\|_{L^{2}}\right\|_{l^{2}(\tau)}^{2}+\varepsilon \sqrt{\partial_{\tau} E_{\omega}(t, \tau)} \sqrt{\partial_{\tau} E_{g}^{2}(t, \tau)}
\end{aligned}
$$


Note that we have performed an integration by parts to go from the first to the second line. The second term can be absorbed for small $\eta$ by the tangential dissipative term at the left-hand side. As regards the first term, we use that

$$
\begin{aligned}
& \varepsilon\left\|j^{3 / 4}\right\| \partial_{x}^{j} \omega\left\|_{L^{2}}\right\|_{l^{2}(\tau)}^{2} \\
& \quad \lesssim \varepsilon\left(\|\| j^{3 / 4} \partial_{x} h_{j-1}\left\|_{L^{2}}\right\|_{l^{2}(\tau)}^{2}+\left\|j^{3 / 4} \partial_{x} g_{j-1}\right\|_{L^{2}}\left\|_{l^{2}(\tau)}^{2}+\right\|\left\|j^{3 / 4} \partial_{x}^{j-1} \omega\right\|_{L^{2}} \|_{l^{2}(\tau)}^{2}\right) \\
& \quad \lesssim \varepsilon\left(\|\| \partial_{x} h_{j}\left\|_{L^{2}}\right\|_{l^{2}(\tau)}^{2}+\left\|\partial_{x} g_{j}\right\|_{L^{2}}\left\|_{l^{2}(\tau)}^{2}+\right\|\left\|\partial_{x}^{j} \omega\right\|_{L^{2}} \|_{l^{2}(\tau)}^{2}\right) .
\end{aligned}
$$

Such inequality can be obtained along the lines of Lemma 2, Eventually, multiplying by a small constant $\alpha$ the energy estimate on $E_{g}^{2}$, this term will be absorbed by the tangential dissipative terms related to $E_{h}$ and $E_{g}^{1}$. For the sake of brevity, we skip the details.

\section{Uniqueness}

In this section, we prove the uniqueness of the solution constructed in the previous section. As usual, uniqueness can be derived by some energy estimate in a space weaker than the space where existence was proved. One can hope to do it in $L^{2}$. However, due to the loss of derivative in the equation, we have to do it with the same Gevrey regularity. We will only use a small loss of Sobolev correction.

We assume that we have two solutions $u^{1}$ and $u^{2}$ of our system (1.3), such that the functionals $\mathcal{E}^{1}\left(\alpha^{0}, t, \tau^{0}\right)$ and $\mathcal{E}^{2}\left(\alpha^{0}, t, \tau^{0}\right)$, defined as in (2.7) with $\omega$ replaced by $\omega^{1}$ and $\omega^{2}$, are bounded (in this whole section, $\omega^{2}$ should not be confused with the square of $\omega$ ). Here, we can take $\alpha^{0}=1$ and $\tau^{0}=\min \left(\tau^{1}, \tau^{2}\right)$. We also assume the lower bound and the upper bound (3.1) to hold for both solutions $\omega^{1}$ and $\omega^{2}$. Finally, we assume the existence of critical curves $a^{1}(t, x)$ and $a^{2}(t, x)$ such that for $i=1,2$, we have

$$
\partial_{t} a^{i}(t, x)+\frac{\partial_{t} \omega^{i}(t, x, a(t, x))}{\partial_{y} \omega^{i}(t, x, a(t, x))}=0, \quad a^{i}(0, x)=a_{0}(x) .
$$

We also recall that $u^{1}$ and $u^{2}$ should remain convex: $\partial_{y}^{2} u^{i}=\partial_{y} \omega^{i}>0$, for $t$ and $y-a^{i}(t, x)$ small enough.

Let us denote $u=u^{1}-u^{2}, v=v^{1}-v^{2}$ and $\omega=\omega^{1}-\omega^{2}$. Hence, we have

$$
\begin{aligned}
& \partial_{t} u+u^{1} \partial_{x} u+v^{1} \partial_{y} u+u \partial_{x} u^{2}+v \partial_{y} u^{2}-\partial_{y}^{2} u=0, \\
& \partial_{t} \omega+u^{1} \partial_{x} \omega+v^{1} \partial_{y} \omega+u \partial_{x} \omega^{2}+v \partial_{y} \omega^{2}-\partial_{y}^{2} \omega=0 .
\end{aligned}
$$

We are going to perform the same type of estimates as in the existence part: the relevant energy, still called $\mathcal{E}(\alpha, t, \tau)$, is defined as in (2.7), but the definition of $h_{j}, g_{j}$ and $\tilde{g}_{j}$ used to define $E_{h}, E_{g}^{1}$ and $E_{g}^{2}$ need to be changed. Indeed, we take

$$
\begin{gathered}
h_{j}(t, x, y)=\chi\left(y-a^{2}(t, x)\right) \frac{\partial_{x}^{j} \omega}{\sqrt{\partial_{y} \omega^{2}}}(t, x, y), \\
g_{j}(t, x, y):=\left(\psi(y) \omega^{2}(t, x, y)+1-\psi(y)\right)\left(\partial_{x}^{j} \omega-\frac{\partial_{y} \omega^{2}}{\omega^{2}} \partial_{x}^{j} u\right)(t, x, y),
\end{gathered}
$$




$$
\tilde{g}_{j}(t, x, y):=\partial_{x}^{j-5}\left(\omega^{2} \partial_{x}^{5} \omega-\partial_{y} \omega^{2} \partial_{x}^{5} u\right) .
$$

with $\psi=\psi(y) \in C_{c}^{\infty}(\mathbb{R})$ equal to 1 in an open neighborhood of $\left[0, \sup \left|a^{2}\right|\right]$. Also, we replace the constraint $j_{2} \leq s$ in the definition of $\dot{E}_{\omega}$ by $j_{2} \leq s-2$. Finally, in all energies, we replace the Sobolev correction term, namely the factor $(j+1)^{10}$, by $(j+1)^{8}$.

Now, we follow the strategy used in the proof of the a priori estimates. We do not give all the details, but just mention the main changes we need to make.

\subsection{Estimate of the vorticity energy}

We differentiate the vorticity equation (5.3) $J$ times, $J=\left(j_{1}, j_{2}\right) \in \mathbb{N}^{2}, \quad 0<j_{2} \leq s-2$. We find, for $\omega_{J}:=\partial^{J} \omega$ :

$$
\begin{array}{r}
\partial_{t} \omega_{J}+u^{1} \partial_{x} \omega_{J}+v^{1} \partial_{y} \omega_{J}-\partial_{y}^{2} \omega_{J}+\left[\partial^{J}, u^{1}\right] \partial_{x} \omega+\left[\partial^{J}, v^{1}\right] \partial_{y} \omega \\
+\partial^{J}\left(u \partial_{x} \omega^{2}+v \partial_{y} \omega^{2}\right)
\end{array}
$$

Arguing as in the proof of Proposition 1, we deduce that

$$
\partial_{t} \dot{E}_{\omega}(t, \tau)+\dot{D}_{\omega}(t, \tau) \lesssim C \partial_{\tau} E_{\omega}(t, \tau) .
$$

We only point out the presence of the extra terms $\partial^{J}\left(u \partial_{x} \omega_{J}^{2}+v \partial_{y} \omega_{J}^{2}\right)$ which can be treated along the estimates of $A_{j}$ and $B_{j}$ in paragraph 3.2. Note here that $u$ and $v$ can only be hit by $J$ derivatives. Moreover, due to the difference between the Sobolev correction factors $(j+1)^{8}$ and $(j+1)^{10}$, we can estimate $\partial^{J} \partial_{x} \omega_{J}^{2}$ and $\partial^{J} \partial_{y} \omega_{J}^{2}$ by the new $\mathcal{E}^{2}$, despite the extra $x$ and $y$ derivative. This is the reason we took a slight loss in Sobolev correction. Also, thanks to the new restriction $j_{2} \leq s-2$, not more than $s$ derivatives with respect to $y$ hit $\omega^{2}$.

\subsection{Estimate of the hydrostatic energy}

As in subsection (3.3), we write an equation for $h_{j}$. It is now:

$$
\begin{aligned}
\partial_{t} h_{j}+u^{1} \partial_{x} h_{j}+v^{1} \partial_{y} h_{j}-\partial_{y}^{2} h_{j} & =\frac{\chi^{a}}{\sqrt{\partial_{y} \omega^{2}}}\left[\partial_{x}^{j}, u^{1}\right] \partial_{x} \omega+\frac{\chi^{a}}{\sqrt{\partial_{y} \omega^{2}}}\left[\partial_{x}^{j}, v^{1}\right] \partial_{y} \omega \\
& +\left[\frac{\chi^{a}}{\sqrt{\partial_{y} \omega^{2}}}, \partial_{t}+u^{1} \partial_{x}+v^{1} \partial_{y}-\partial_{y}^{2}\right] \partial_{x}^{j} \omega-\frac{\chi^{a}}{\sqrt{\partial_{y} \omega^{2}}} \partial_{x}^{j} v \partial_{y} \omega^{2} \\
& -\frac{\chi^{a}}{\sqrt{\partial_{y} \omega^{2}}} \partial_{x}^{j}\left(u \partial_{x} \omega^{2}\right)-\frac{\chi^{a}}{\sqrt{\partial_{y} \omega^{2}}}\left[\partial_{x}^{j}, \partial_{y} \omega^{2}\right] v
\end{aligned}
$$

Performing a standard energy estimate on the previous equation, multiplying by $\left(\tau^{j}(j !)^{-7 / 4} j^{8}\right)^{2}$, summing over $j$, we end up with and equation similar to (3.31). The main term now is

$$
E_{j}:=\int_{\mathbb{T} \times \mathbb{R}_{+}} \frac{\chi^{a}}{\sqrt{\partial_{y} \omega^{2}}} \partial_{x}^{j} v \partial_{y} \omega^{2} h_{j}
$$

which can be treated exactly as in subsection 3.3 . Hence, we deduce that the estimate (3.30) holds with the new definition of $u, \omega$ and with the change of the Sobolev correction in the energies. 


\subsection{Estimate of the (second) monotonicity energy}

As in subsection (3.3), we apply $\omega^{2} \partial_{x}^{5}$ to the vorticity equation (5.3), $\partial_{y} \omega^{2} \partial_{x}^{5}$ to the velocity equation (5.2), and substract one from the other:

$$
\begin{aligned}
\partial_{t} \tilde{g}_{5}+u^{1} \partial_{x} \tilde{g}_{5}+v^{1} \partial_{y} \tilde{g}_{5}-\partial_{y}^{2} \tilde{g}_{5}= & -\sum_{k=1}^{5}\left(\begin{array}{l}
5 \\
k
\end{array}\right) \partial_{x}^{k} u^{1} \bar{g}_{5-k+1}-\sum_{k=1}^{5}\left(\begin{array}{l}
5 \\
k
\end{array}\right) \partial_{x}^{k} v^{1} \hat{g}_{5-k+1} \\
& -\sum_{k=0}^{5}\left(\begin{array}{l}
5 \\
k
\end{array}\right) \partial_{x}^{k} u \bar{g}_{5-k+1}^{2}-\sum_{k=0}^{4}\left(\begin{array}{l}
5 \\
k
\end{array}\right) \partial_{x}^{k} v \hat{g}_{5-k+1}^{2} \\
& -\left(\partial_{t}+u^{1} \partial_{x}+v^{1} \partial_{y}-\partial_{y}^{2}\right) \partial_{y} \omega^{2} \partial_{x}^{5} u+2 \partial_{y}^{2} \omega^{2} \partial_{x}^{5} \omega-2 \partial_{y} \omega^{2} \partial_{y} \partial_{x}^{5} \omega \\
& +\left(\partial_{t}+u^{1} \partial_{x}+v^{1} \partial_{y}-\partial_{y}^{2}\right) \omega^{2} \partial_{x}^{5} \omega
\end{aligned}
$$

with $\hat{g}_{k}, \bar{g}_{k}, \hat{g}_{k}^{2}, \bar{g}_{k}^{2}$ defined as in (3.51), for instance $\bar{g}_{k}:=\omega^{2} \partial_{x}^{k} \omega-\partial_{y} \omega^{2} \partial_{x}^{k} u$ and $\bar{g}_{k}^{2}:=$ $\omega^{2} \partial_{x}^{k} \omega^{2}-\partial_{y} \omega^{2} \partial_{x}^{k} u^{2}$ for $k \leq 5$. Note that the term that caused a loss of one $x$ derivative has disappeared. As in the proof of Proposition [3, we apply $\partial_{x}^{j-5}$ to the equation (5.7) and we conclude in a similar way that (3.48) holds.

\subsection{Estimate of the (first) monotonicity energy}

As in the proof of Proposition 4, we write an equation for $g_{j}$ defined in (5.5):

$$
\begin{aligned}
& \partial_{t} g_{j}+u^{1} \partial_{x} g_{j}+v^{1} \partial_{y} g_{j}-\partial_{y}^{2} g_{j} \\
& =-\sum_{k=1}^{j}\left(\begin{array}{l}
j \\
k
\end{array}\right) \partial_{x}^{k} u^{1} g_{j-k+1}-\sum_{k=1}^{j}\left(\begin{array}{l}
j \\
k
\end{array}\right) \partial_{x}^{k} v^{1} \tilde{\psi}\left(\partial_{y} \partial_{x}^{j-k} \omega-\frac{\partial_{y} \omega^{2}}{\omega^{2}} \partial_{x}^{j-k} \omega\right) \\
& -\sum_{k=0}^{j}\left(\begin{array}{l}
j \\
k
\end{array}\right) \partial_{x}^{k} u g_{j-k+1}^{2}-\sum_{k=0}^{j-1}\left(\begin{array}{l}
j \\
k
\end{array}\right) \partial_{x}^{k} v \tilde{\psi}\left(\partial_{y} \partial_{x}^{j-k} \omega^{2}-\frac{\partial_{y} \omega^{2}}{\omega^{2}} \partial_{x}^{j-k} \omega^{2}\right) \\
& +\left(\partial_{t}+u^{1} \partial_{x}+v^{1} \partial_{y}-\partial_{y}^{2}\right) \tilde{\psi} \partial_{x}^{j} \omega-\left(\partial_{t}+u^{1} \partial_{x}+v^{1} \partial_{y}-\partial_{y}^{2}\right)\left(\tilde{\psi} \frac{\partial_{y} \omega^{2}}{\omega^{2}}\right) \partial_{x}^{j} u \\
& -2 \partial_{y} \tilde{\psi} \partial_{y} \partial_{x}^{j} \omega+2 \partial_{y}\left(\tilde{\psi} \frac{\partial_{y} \omega}{\omega}\right) \partial_{x}^{j} \omega
\end{aligned}
$$

where $\tilde{\psi}=\left(\psi(y) \omega^{2}(t, x, y)+1-\psi(y)\right)$. Again the main thing here is that in the third line the term that had a potential loss of $x$ derivative, namely the one involving $\partial_{x}^{j} v$ was canceled. As above, we get an estimate similar to (3.70).

Putting all these estimates together, we can easily conclude, as in the a priori estimate section, by a Gronwall lemma that $u=0$.

\section{Conclusions}

We have proved in this paper the local wellposedness of the Prandtl system for data that are in the Gevrey class $7 / 4$ in the horizontal variable $x$. We would like to discuss here some possible extension of this work: 
- First, it is unlikely that the Gevrey class $7 / 4$ is optimal. In particular, the analysis and numerics performed in [6] (on a simple linearization) suggest that the optimal exponent may be $s=2$. However, we believe that extending our result to the Gevrey class 2 or even $2-\varepsilon$ for all $\varepsilon>0$ would require some new ideas. Moreover, it remains uncertain that the index $s=2$ is the critical one since there may be more severe instabilities than those constructed in $[6]$.

- For simplicity, we have considered here homogeneous source terms: $U=0$ and $P=0$. However, our work should extend to the case where $U$ is a function of $t, x$, along the lines of [13. Also, we have considered data that have polynomial decay when $y$ goes to infinity. One can adapt the proof to data with exponential decay, using some Hardy type inequality with exponential weights.

- An important open question is to prove global existence for small data for the Prandtl system.

- Finally, we recall that the study of the Prandtl system is motivated by the zero viscosity limit in the presence of a boundary. The present work opens the road to a full justification of the Euler-Prandtl description, without analyticity.

\section{A Sobolev and Hardy inequalities}

We recall classical Sobolev and Hardy inequalities, see [12]: For $f=f(x, y)$, we have

$$
\|f\|_{L^{\infty}} \lesssim\|f\|_{L^{2}}+\left\|\partial_{x} f\right\|_{L^{2}}+\left\|\partial_{y} f\right\|_{L^{2}}+\left\|\partial_{y} \partial_{x} f\right\|_{L^{2}}
$$

If $\lambda>-\frac{1}{2}$ and $\lim _{y \rightarrow+\infty} f(x, y)=0$, then

$$
\left\|(1+y)^{\lambda} f\right\|_{L^{2}} \leq \frac{2}{2 \lambda+1}\left\|(1+y)^{\lambda+1} \partial_{y} f\right\|_{L^{2}}
$$

If $\lambda<-\frac{1}{2}$, we have

$$
\left\|(1+y)^{\lambda} f\right\|_{L^{2}} \leq \sqrt{-\frac{1}{2 \lambda+1}}\left\|\left.f\right|_{y=0}\right\|_{L^{2}}-\frac{2}{2 \lambda+1}\left\|(1+y)^{\lambda+1} \partial_{y} f\right\|_{L^{2}}
$$

\section{B Proof of technical lemmas}

\section{B.1 Proof of Lemma 2}

The point is to prove that

$$
\left\|\partial_{x}^{j} \omega\right\|_{L_{\gamma}^{2}} \lesssim\left\|h_{j}\right\|_{L^{2}\left(\mathbb{T} \times \mathbb{R}_{+}\right)}+\left\|g_{j}\right\|_{L_{\gamma}^{2}} \lesssim\left\|\partial_{x}^{j} \omega\right\|_{L_{\gamma}^{2}}
$$

Inequality on the right. Clearly, $\left\|h_{j}\right\|_{L^{2}\left(\mathbb{T} \times \mathbb{R}_{+}\right)} \lesssim\left\|\partial_{x}^{j} \omega\right\|_{L_{\gamma}^{2}}$. Then, by assumption (3.1),

$$
(1+y)^{\gamma}\left|g_{j}\right| \lesssim(1+y)^{\gamma}\left|\partial_{x}^{j} \omega\right|+(1+y)^{\gamma-1}\left|\partial_{x}^{j} u\right|
$$


and from Hardy inequality (啇), we deduce: $\left\|g_{j}\right\|_{L_{\gamma}^{2}} \lesssim\left\|\partial_{x}^{j} \omega\right\|_{L_{\gamma}^{2}}$.

Inequality on the left. First, from the definition of $h_{j}$,

$$
\left\|\partial_{x}^{j} \omega\right\|_{L^{2}(\{|y-a|<\varepsilon\})} \lesssim\left\|h_{j}\right\|_{L^{2}\left(\mathbb{T} \times \mathbb{R}_{+}\right)}
$$

for $\varepsilon>0$ small enough so that $\{|y-a|<\varepsilon\} \subset\{\chi=1\}$. Then, we use the identities stated in Lemma 3 .

$$
\partial_{x}^{j} u(t, x, y)=\left\{\begin{array}{l}
\omega(t, x, y) \int_{3}^{y}\left(\psi+\frac{(1-\psi)}{\omega}\right)^{-1} \frac{g_{j}}{\omega^{2}}+C_{j}(t, x) \omega(t, x, y), \quad y>a(t, x), \\
\omega(t, x, y) \int_{0}^{y}\left(\psi+\frac{(1-\psi)}{\omega}\right)^{-1} \frac{g_{j}}{\omega^{2}}, \quad y<a(t, x)
\end{array}\right.
$$

We integrate the first equality for $x \in \mathbb{T}, \frac{\varepsilon}{2}<y-a<\varepsilon$. As $\omega$ does not vanish in this region, it allows to obtain a control of $C_{j}$ :

$$
\begin{aligned}
\left\|C_{j}\right\|_{L^{2}(\mathbb{T})} & \leq C_{\varepsilon}\left(\left\|\partial_{x}^{j} u\right\|_{L^{2}\left(\left\{\frac{\varepsilon}{2}<y-a<\varepsilon\right\}\right)}+\left\|g_{j}\right\|_{\left.L^{2}\left(\left\{\frac{\varepsilon}{2}<y-a<\varepsilon\right\}\right\}\right)}\right) \\
& \leq C_{\varepsilon}^{\prime}\left(\left\|\partial_{x}^{j} \omega\right\|_{L^{2}(\{0<y<\varepsilon+a\})}+\left\|g_{j}\right\|_{L^{2}(\{y<3\})}\right) \\
& \leq C_{\varepsilon}^{\prime}\left(\left\|\partial_{x}^{j} \omega\right\|_{L^{2}(\{|y-a|<\varepsilon\})}+\left\|\partial_{x}^{j} \omega\right\|_{L^{2}(\{y-a<-\varepsilon\})}+\left\|g_{j}\right\|_{L^{2}(\{y<3\})}\right) \\
& \leq C_{\varepsilon}^{\prime \prime}\left(\left\|h_{j}\right\|_{L^{2}\left(\mathbb{T} \times \mathbb{R}_{+}\right)}+\left\|g_{j}\right\|_{L^{2}\left(L_{\gamma}^{2}\right)}\right) .
\end{aligned}
$$

Finally, considering identities (B.3) for $|y-a| \geq \varepsilon$, we get

$$
\left\|(1+y)^{\gamma} \partial_{x}^{j} \omega\right\|_{L^{2}(\{|y-a| \geq \varepsilon\})} \leq C_{\varepsilon}\left(\left\|C_{j}\right\|_{L^{2}(\mathbb{T})}+\left\|g_{j}\right\|_{L^{2}\left(L_{\gamma}^{2}\right)}\right) \leq C_{\varepsilon}\left(\left\|h_{j}\right\|_{L^{2}\left(\mathbb{T} \times \mathbb{R}_{+}\right)}+\left\|g_{j}\right\|_{L^{2}\left(L_{\gamma}^{2}\right)}\right)
$$

where the last inequality comes from (B.4). Combining with (B.2) yields the result.

\section{B.2 Proof of Lemma 6}

Bound on $u_{g}^{j}$. We denote $\psi_{\omega}:=(\psi+(1-\psi) / \omega)^{-1}$. For $a-\varepsilon \leq y \leq 3$

$$
\begin{array}{r}
\int_{0}^{y} u_{g}^{j}=\int_{0}^{a} \omega \int_{0}^{y} \frac{g \psi_{\omega}}{\omega^{2}}+\int_{a}^{y} \omega \int_{3}^{y} \frac{g \psi_{\omega}}{\omega^{2}}=\int_{0}^{a} \frac{\left.u\right|_{y=a}-u}{\omega^{2}} g \psi_{\omega}+\int_{a}^{y} \frac{\left.u\right|_{y=a}-u}{\omega^{2}} g \psi_{\omega} \\
+\left(\left.u\right|_{y=a}-u(\cdot, y)\right) \int_{3}^{y} \frac{g \psi_{\omega}}{\omega^{2}}
\end{array}
$$

after integration by parts. The third term at the right-hand side does not raise any problem, as the integral only involves values of $y \varepsilon$-away from $y=a$. For the third and second term, we notice that

$$
\left|\frac{u-\left.u\right|_{y=a}}{\omega^{2}}\right| \leq C \quad \text { for } 0 \leq y \leq 3 .
$$

Finally, we get

$$
\left|\int_{0}^{y} u_{g}^{j}\right| \lesssim \int_{0}^{3}|g|
$$

and the bound follows easily. 
Bound on $C_{j}$. From the proof of Lemma $2(c f(\underline{B .4}))$, we know that

$$
\left\|C_{j}\right\|_{L^{2}(\mathbb{T})} \lesssim\left\|h_{j}\right\|_{L^{2}\left(\mathbb{T} \times \mathbb{R}_{+}\right)}+\left\|g_{j}\right\|_{L^{2}\left(L_{\gamma}^{2}\right)} \lesssim\left\|\partial_{x}^{j} \omega\right\|_{L^{2}\left(L_{\gamma}^{2}\right)} .
$$

Bound on $\partial_{x} C_{j}$ : We write: for $y>a$,

$$
\omega \partial_{x} C_{j}=\partial_{x}^{j+1} u-\partial_{x} \omega C_{j}-\partial_{x}\left(\omega \int_{3}^{y} \frac{g_{j} \psi_{\omega}}{\omega^{2}}\right)
$$

As for $C_{j}$, we integrate for $\frac{\varepsilon}{2} \leq y \leq \varepsilon, \varepsilon>0$ small enough. We end up with

$$
\left\|\partial_{x} C_{j}\right\|_{L^{2}(\mathbb{T})} \lesssim\left\|C_{j}\right\|_{L^{2}(\mathbb{T})}+\left\|\partial_{x}^{j+1} \omega\right\|_{L^{2}\left(L_{\gamma}^{2}\right)}+\left\|g_{j}\right\|_{L^{2}(\{y<3\})}+\left\|\partial_{x} g_{j}\right\|_{L^{2}(\{y<3\})}
$$

Easily, the last two terms are bounded by $\left\|\partial_{x}^{j} \omega\right\|_{L^{2}\left(L_{\gamma}^{2}\right)}+\left\|\partial_{x}^{j+1} \omega\right\|_{L^{2}\left(L_{\gamma}^{2}\right)}$. The result follows.

\section{B.3 Proof of Lemma 7}

It is enough to prove that for all $\alpha \geq 0$,

$$
\|\| j^{\alpha / 4}\left(\tilde{g}_{j}-g_{j}\right)\left\|_{L^{2}(\{y \leq 3\})}\right\|_{l^{2}(\tau)} \lesssim\left\|j^{(\alpha-3) / 4}\right\| \omega\left\|_{\mathcal{H}_{\gamma}^{j}}\right\|_{l^{2}(\tau)}
$$

For $y \in[0,3]$, we have

$$
\begin{aligned}
\tilde{g}_{j}-g_{j} & =\left[\partial_{x}^{j-5}, \omega\right] \partial_{x}^{5} \omega-\left[\partial_{x}^{j-5}, \partial_{y} \omega\right] \partial_{x}^{5} u \\
& =\sum_{k=1}^{j-5}\left(\begin{array}{c}
j-5 \\
k
\end{array}\right) \partial_{x}^{k} \omega \partial_{x}^{j-k} \omega-\partial_{x}^{k} \partial_{y} \omega \partial_{x}^{j-k} u
\end{aligned}
$$

This kind of sums has been considered several times throughout the paper. We get here

$$
\begin{aligned}
\left\|\tilde{g}_{j}-g_{j}\right\|_{L^{2}(\{y \leq 3\}) \leq} & \sum_{k=1}^{\frac{j-5}{2}}\left(\begin{array}{c}
j-5 \\
k
\end{array}\right) \sum_{m=0}^{3}\|\omega\|_{\mathcal{H}_{\gamma}^{k+m}}\|\omega\|_{\mathcal{H}_{\gamma}^{j-k}} \\
& +\sum_{k=\frac{j-5}{2}}^{j-5}\left(\begin{array}{c}
j-5 \\
k
\end{array}\right)\left(\|\omega\|_{\mathcal{H}_{\gamma}^{k}}+\|\omega\|_{\mathcal{H}_{\gamma}^{k+1}}\right) \sum_{m=0}^{2}\|\omega\|_{\mathcal{H}_{\gamma}^{j-k+m}}:=A_{j}+B_{j} .
\end{aligned}
$$

We focus on $A_{j}$, as the other term $B_{j}$ is much better. Through the change of index $k^{\prime}:=k-1$, we find that

$$
\begin{aligned}
A_{j} & \lesssim \sum_{k=0}^{\frac{j-5}{2}-1}\left(\begin{array}{c}
j-5 \\
k+1
\end{array}\right) \sum_{m=1}^{4}\|\omega\|_{\mathcal{H}_{\gamma}^{k+m}}\|\omega\|_{\mathcal{H}_{\gamma}^{j-1-k}} \\
& \lesssim \sum_{k=0}^{\frac{j-1}{2}}\left(\begin{array}{c}
j-1 \\
k
\end{array}\right) \sum_{m=1}^{4}\|\omega\|_{\mathcal{H}_{\gamma}^{k+m}}(j-1-k)\|\omega\|_{\mathcal{H}_{\gamma}^{j-1-k}}
\end{aligned}
$$

using the inequality $\left(\begin{array}{c}j \\ k\end{array}\right) \lesssim\left(\begin{array}{c}j-1 \\ k\end{array}\right) j \lesssim\left(\begin{array}{c}j-1 \\ k\end{array}\right)(j-1-k)$. It follows that

$$
\left\|j^{\gamma / 4} A_{j}\right\|_{l^{2}(\tau)} \lesssim\left\|j^{\gamma / 4} j\right\| \omega\left\|_{\mathcal{H}_{\gamma}^{j-1}}\right\|_{l^{2}(\tau)} \leq\left\|j^{(\gamma-3) / 4}\right\| \omega\left\|_{\mathcal{H}_{\gamma}^{j}}\right\|_{l^{2}(\tau)} .
$$

This leads to the expected bound. 


\section{B.4 Proof of Lemma 8}

We only treat the case $l=1$ :

$$
\|\| j^{\alpha} \partial_{y} \bar{g}_{j-1}\left\|_{L^{2}\left(\mathbb{T} \times \mathbb{R}_{+}\right)}\right\|_{l^{2}(\tau)} \lesssim\left\|j^{\alpha}\right\| \omega\left\|_{\mathcal{H}_{\gamma}^{j}}\right\|_{l^{2}(\tau)} .
$$

The other cases $l=0$ and $l=2$ are handled in the same way. We compute

$$
\begin{aligned}
\partial_{y} \tilde{g}_{j-1} & =\partial_{x}^{j-6}\left(\omega \partial_{y} \partial_{x}^{5} \omega-\partial_{y}^{2} \omega \partial_{x}^{5} u\right) \\
& =\sum_{k=0}^{j-6}\left(\begin{array}{c}
j-6 \\
k
\end{array}\right) \partial_{x}^{k} \omega \partial_{y} \partial_{x}^{j-k-1} \omega-\sum_{k=0}^{j-6}\left(\begin{array}{c}
j-6 \\
k
\end{array}\right) \partial_{x}^{k} \partial_{y}^{2} \omega \partial_{x}^{j-k-1} u
\end{aligned}
$$

By now standard computations:

$$
\begin{aligned}
\left\|\partial_{y} \tilde{g}_{j-1}\right\|_{L^{2}\left(\mathbb{T} \times \mathbb{R}_{+}\right)} \lesssim & \sum_{k=0}^{\frac{j-6}{2}}\left(\begin{array}{c}
j-6 \\
k
\end{array}\right) \sum_{m=0}^{4}\|\omega\|_{\mathcal{H}_{\gamma}^{k+m}}\left(\|\omega\|_{\mathcal{H}_{\gamma}^{j-k}}+\|\omega\|_{\mathcal{H}_{\gamma}^{j-1-k}}\right) \\
& +\sum_{\frac{j-6}{2}}^{j-6}\left(\begin{array}{c}
j-6 \\
k
\end{array}\right)\left(\|\omega\|_{\mathcal{H}_{\gamma}^{k}}+\|\omega\|_{\mathcal{H}_{\gamma}^{k+2}}\right) \sum_{m=-1}^{2}\|\omega\|_{\mathcal{H}^{j-k+m}}:=A_{j}+B_{j} .
\end{aligned}
$$

On one hand, as $\left(\begin{array}{c}j-6 \\ k\end{array}\right) \lesssim\left(\begin{array}{l}j \\ k\end{array}\right)$, Lemma 1 clearly implies

$$
\left\|j^{\alpha} A_{j}\right\|_{l^{2}(\tau)} \lesssim\left\|j^{\alpha}\right\| \omega\left\|_{\mathcal{H}_{\gamma}^{j}}\right\|_{l^{2}(\tau)} .
$$

On the other hand, after the change of index $k^{\prime}:=k+2$

$$
B_{j} \lesssim \sum_{k=\frac{j}{2}}^{j}\left(\begin{array}{l}
j \\
k
\end{array}\right)\left(\|\omega\|_{\mathcal{H}_{\gamma}^{k-2}}+\|\omega\|_{\mathcal{H}_{\gamma}^{k}}\right) \sum_{m=1}^{4}\|\omega\|_{\mathcal{H}^{j-k+m}}
$$

(notice that $\left.\left(\begin{array}{l}j-6 \\ k-2\end{array}\right) \lesssim\left(\begin{array}{l}j \\ k\end{array}\right)\right)$. Still by Lemma 1,

$$
\left\|j^{\alpha} B_{j}\right\|_{l^{2}(\tau)} \lesssim\left\|j^{\alpha}\right\| \omega\left\|_{\mathcal{H}_{\gamma}^{j}}\right\|_{l^{2}(\tau)} .
$$

Combining the bounds on $A_{j}$ and $B_{j}$ yields the result.

\section{B.5 Proof of Lemma 9}

For $(x, y)$ in the support of $\chi^{\varepsilon, a}$, we have

$$
\partial_{y}\left(\tilde{g}_{j}-g_{j}\right)=\sum_{k=1}^{j-5}\left(\begin{array}{c}
j-5 \\
k
\end{array}\right)\left(\partial_{x}^{k} \omega \partial_{x}^{j-k} \partial_{y} \omega-\partial_{x}^{k} \partial_{y}^{2} \omega \partial_{x}^{j-k} u\right) .
$$

We proceed as we did several times in the paper: we get

$$
\begin{aligned}
& \left\|\sqrt{\chi^{\varepsilon, a}} \partial_{y}\left(\tilde{g}_{j}-g_{j}\right)\right\|_{L^{2}\left(\mathbb{T} \times \mathbb{R}_{+}\right)} \\
& \lesssim \sum_{k=1}^{\frac{j-5}{2}} \sum_{m=0}^{4}\|\omega\|_{\mathcal{H}_{\gamma}^{k+m}}\left(\left\|\sqrt{\chi^{\varepsilon, a}} \partial_{x}^{j-k} \partial_{y} \omega\right\|_{L^{2}\left(\mathbb{T} \times \mathbb{R}_{+}\right)}+\|\omega\|_{\mathcal{H}_{\gamma}^{j-k}}\right) \\
& +\sum_{k=\frac{j-5}{2}}^{j-5}\left(\begin{array}{c}
j-5 \\
k
\end{array}\right)\left(\|\omega\|_{\mathcal{H}_{\gamma}^{k}} \sum_{m=1}^{3}\|\omega\|_{\mathcal{H}_{\gamma}^{j-k+m}}+\|\omega\|_{\mathcal{H}_{\gamma}^{k+2}} \sum_{m=0}^{2}\|\omega\|_{\mathcal{H}_{\gamma}^{j-k+m}}\right) \\
& :=A_{j}+B_{j} .
\end{aligned}
$$


Note that we did not bound $\left\|\sqrt{\chi^{\varepsilon, a}} \partial_{x}^{j-k} \partial_{y} \omega\right\|_{L^{2}}$ by $\left\|\partial_{y} \omega\right\|_{\mathcal{H}_{\gamma}^{j-k}}$ : we need to keep track of the localization to handle this term.

Treatment of $A_{j}$. We set $k^{\prime}:=k-1$. Dropping the prime,

$$
\begin{aligned}
A_{j} & \lesssim \sum_{k=0}^{\frac{j-5}{2}-1}\left(\begin{array}{c}
j-5 \\
k+1
\end{array}\right) \sum_{m=1}^{5}\|\omega\|_{\mathcal{H}_{\gamma}^{k+m}}\left(\left\|\sqrt{\chi^{\varepsilon, a}} \partial_{x}^{j-1-k} \partial_{y} \omega\right\|_{L^{2}\left(\mathbb{T} \times \mathbb{R}_{+}\right)}+\|\omega\|_{\mathcal{H}_{\gamma}^{j-1-k}}\right) \\
& \lesssim \sum_{k=0}^{\frac{j-1}{2}}\left(\begin{array}{c}
j-1 \\
k
\end{array}\right) \sum_{m=0}^{5}\|\omega\|_{\mathcal{H}_{\gamma}^{k+m}}(j-1-k)\left(\left\|\sqrt{\chi^{\varepsilon, a}} \partial_{x}^{j-1-k} \partial_{y} \omega\right\|_{L^{2}\left(\mathbb{T} \times \mathbb{R}_{+}\right)}+\|\omega\|_{\mathcal{H}_{\gamma}^{j-1-k}}\right)
\end{aligned}
$$

Here, we have used $\left(\begin{array}{c}j-5 \\ k+1\end{array}\right) \lesssim j\left(\begin{array}{c}j-1 \\ k\end{array}\right) \lesssim(j-1-k)\left(\begin{array}{c}j-1 \\ k\end{array}\right)$. By lemma 1, we find

$$
\begin{aligned}
\left\|j^{3 / 4} A_{j}\right\|_{l^{2}(\tau)} & \lesssim\|\| \omega\left\|_{\mathcal{H}_{\gamma}^{j}}\right\|_{l^{2}(\tau)}\left(\left\|j^{7 / 4}\right\| \sqrt{\chi^{\varepsilon, a}} \partial_{x}^{j-1} \partial_{y} \omega\left\|_{L^{2}\left(\mathbb{T} \times \mathbb{R}_{+}\right)}\right\|_{l^{2}(\tau)}+\left\|j^{7 / 4}\right\| \omega\left\|_{\mathcal{H}_{\gamma}^{j-1}}\right\|_{l^{2}(\tau)}\right) \\
& \lesssim\|\| \omega\left\|_{\mathcal{H}_{\gamma}^{j}}\right\|_{l^{2}(\tau)}\left(\|\| \sqrt{\chi^{\varepsilon, a}} \partial_{x}^{j} \partial_{y} \omega\left\|_{L^{2}\left(\mathbb{T} \times \mathbb{R}_{+}\right)}\right\|_{l^{2}(\tau)}+\|\| \omega\left\|_{\mathcal{H}_{\gamma}^{j}}\right\|_{l^{2}(\tau)}\right) .
\end{aligned}
$$

Finally, we notice that for $\varepsilon$ small enough:

$$
\left\|\sqrt{\chi^{\varepsilon, a}} \partial_{x}^{j} \partial_{y} \omega\right\|_{L^{2}\left(\mathbb{T} \times \mathbb{R}_{+}\right)} \lesssim\left\|\partial_{y} h_{j}\right\|_{L^{2}\left(\mathbb{T} \times \mathbb{R}_{+}\right)}+\left\|\partial_{x}^{j} \omega\right\|_{L^{2}\left(\mathbb{T} \times \mathbb{R}_{+}\right)}
$$

so that

$$
\left\|j^{3 / 4} A_{j}\right\|_{l^{2}(\tau)} \lesssim \sqrt{E_{\omega}(t, \tau)}\left(\sqrt{D_{h}(t, \tau)}+\sqrt{E_{\omega}(t, \tau)}\right) \lesssim \sqrt{D_{h}(t, \tau)}+\sqrt{E_{\omega}(t, \tau)}
$$

Treatment of $B_{j}$. Through the change of index $k^{\prime}:=k+1$ (first term) and $k^{\prime}:=k+3$ (second term), and using that $\left(\begin{array}{l}j-5 \\ k-d\end{array}\right) \lesssim\left(\begin{array}{l}j \\ k\end{array}\right), d=1$ or 3 , we get:

$$
B_{j} \lesssim \sum_{k=\frac{j}{2}}^{j}\left(\begin{array}{l}
j \\
k
\end{array}\right)\|\omega\|_{\mathcal{H}_{\gamma}^{k-2}} \sum_{m=2}^{5}\|\omega\|_{\mathcal{H}_{\gamma}^{j-k+m}} .
$$

Thus,

$$
\begin{aligned}
\left\|j^{3 / 4} B_{j}\right\|_{l^{2}(\tau)} & \lesssim\|\| \omega\left\|_{\mathcal{H}_{\gamma}^{j}}\right\|_{l^{2}(\tau)}\left\|j^{3 / 4}\right\| \omega\left\|_{\mathcal{H}_{\gamma}^{j-1}}\right\|_{l^{2}(\tau)} \\
& \lesssim\|\| \omega\left\|_{\mathcal{H}_{\gamma}^{j}}\right\|_{l^{2}(\tau)}\left\|j^{-1}\right\| \omega\left\|_{\mathcal{H}_{\gamma}^{j}}\right\|_{l^{2}(\tau)} \lesssim \sqrt{E_{\omega}(t, \tau)} .
\end{aligned}
$$

Combining the bounds on $A_{j}$ and $B_{j}$ yields the result.

\section{References}

[1] R. Alexandre, Y.-G. Wang, C.-J. Xu, and T. Yang. Well-posedness of the Prandtl equation in sobolev spaces. preprint, 2012.

[2] Y. Brenier. Homogeneous hydrostatic flows with convex velocity profiles. Nonlinearity, 12(3):495-512, 1999. 
[3] S. J. Cowley, L. M. Hocking, and O. R. Tutty. The stability of solutions of the classical unsteady boundary-layer equation. Phys. Fluids, 28(2):441-443, 1985.

[4] A. B. Ferrari and E. S. Titi. Gevrey regularity for nonlinear analytic parabolic equations. Comm. Partial Differential Equations, 23(1-2):1-16, 1998.

[5] C. Foias and R. Temam. Gevrey class regularity for the solutions of the Navier-Stokes equations. J. Funct. Anal., 87(2):359-369, 1989.

[6] D. Gérard-Varet and E. Dormy. On the ill-posedness of the Prandtl equation. J. Amer. Math. Soc., 23(2):591-609, 2010.

[7] D. Gérard-Varet and T. Nguyen. Remarks on the ill-posedness of the Prandtl equation. Asymtotic Analysis (to appear), 2012.

[8] E. Grenier. On the derivation of homogeneous hydrostatic equations. M2AN Math. Model. Numer. Anal., 33(5):965-970, 1999.

[9] E. Grenier. On the stability of boundary layers of incompressible Euler equations. $J$. Differential Equations, 164(1):180-222, 2000.

[10] E. Guyon, J. Hulin, and L. Petit. Hydrodynamique physique, volume 142 of EDP Sciences. CNRS Editions, Paris, 2001.

[11] L. Hong and J. K. Hunter. Singularity formation and instability in the unsteady inviscid and viscous Prandtl equations. Commun. Math. Sci., 1(2):293-316, 2003.

[12] A. Kufner, L. Maligranda, and L.-E. Persson. The Hardy inequality. Vydavatelský Servis, Plzeň, 2007. About its history and some related results.

[13] I. Kukavica and V. Vicol. On the local existence of analytic solutions to the Prandtl boundary layer equations. Communications in Mathematical Sciences, 11(1):269-292, 2013.

[14] C. D. Levermore and M. Oliver. Analyticity of solutions for a generalized Euler equation. J. Differential Equations, 133(2):321-339, 1997.

[15] M. C. Lombardo, M. Cannone, and M. Sammartino. Well-posedness of the boundary layer equations. SIAM J. Math. Anal., 35(4):987-1004 (electronic), 2003.

[16] N. Masmoudi. Examples of singular limits in hydrodynamics. In Evolutionary equations. Vol. III, Handb. Differ. Equ., pages 195-276. Elsevier/North-Holland, Amsterdam, 2007.

[17] N. Masmoudi and T. K. Wong. Local-in-time existence and uniqueness of solutions to the Prandtl equations by energy methods. preprint, 2012.

[18] N. Masmoudi and T. K. Wong. On the $H^{s}$ theory of hydrostatic Euler equations. Arch. Ration. Mech. Anal., 204(1):231-271, 2012.

[19] C. Mouhot and C. Villani. On Landau damping. Acta Math., 207(1):29-201, 2011.

[20] O. A. Oleinik and V. N. Samokhin. Mathematical models in boundary layer theory, volume 15 of Applied Mathematics and Mathematical Computation. Chapman \& Hall/CRC, Boca Raton, FL, 1999. 
[21] M. Oliver and E. S. Titi. Analyticity of the attractor and the number of determining nodes for a weakly damped driven nonlinear Schrödinger equation. Indiana Univ. Math. J., 47(1):49-73, 1998.

[22] M. Renardy. Ill-posedness of the hydrostatic Euler and Navier-Stokes equations. Arch. Ration. Mech. Anal., 194(3):877-886, 2009.

[23] M. Sammartino and R. E. Caflisch. Zero viscosity limit for analytic solutions, of the Navier-Stokes equation on a half-space. I. Existence for Euler and Prandtl equations. Comm. Math. Phys., 192(2):433-461, 1998.

[24] Z. Xin and L. Zhang. On the global existence of solutions to the Prandtl's system. Adv. Math., 181(1):88-133, 2004. 